\title{
Synthetic Studies of Neoclerodane Diterpenes from Salvia divinorum: Design, Synthesis, and Evaluation of Analogues with Improved Potency and G-protein Activation Bias at the $\mu$ Opioid Receptor
}

Rachel S. Crowley, ${ }^{1}$ Andrew P. Riley, ${ }^{2}$ Amy F. Alder, ${ }^{3}$ Richard J. Anderson III, ${ }^{3}$ Dan Luo, ${ }^{1,4}$ Sophia Kaska, ${ }^{1,4}$ Pamela Maynez, ${ }^{1}$ Bronwyn M. Kivell, ${ }^{3}$ and Thomas E. Prisinzano ${ }^{1,4, *}$

${ }^{1}$ Department of Medicinal Chemistry, School of Pharmacy, University of Kansas, Lawrence, KS 66047, United States; ${ }^{2}$ Department of Chemistry, University of Kansas, Lawrence, KS 66045, United States; ${ }^{3}$ School of Biological Sciences, Centre for Biodiscovery, Victoria University of Wellington, Wellington 6140, New Zealand; ${ }^{4}$ Department of Pharmaceutical Sciences, College of Pharmacy, University of Kentucky, Lexington, Kentucky

Table of Contents

\begin{tabular}{ll}
\hline Supplemental Table 1 & S2 \\
Chemistry & S3 \\
NMR Spectra & $\mathrm{S} 15$ \\
HPLC Traces & $\mathrm{S} 80$ \\
References & $\mathrm{S} 91$
\end{tabular}


Supplemental Table 1: Calculated properties and ligand efficiencies of kurkinorin derivatives.

\begin{tabular}{|c|c|c|c|c|c|}
\hline Compound & $\operatorname{tPSA}\left(\AA^{2}\right)^{a}$ & $s \log \mathrm{P}^{b}$ & $\mathrm{HAC}^{c}$ & $\begin{array}{c}\text { Ligand } \\
\text { Efficiency }\end{array}$ & LELP $^{e}$ \\
\hline Kurkinorin (1) & 109.1 & 3.8 & 36 & 0.34 & 11.19 \\
\hline 4 & 122 & 3.2 & 36 & 0.24 & 13.16 \\
\hline 5 & 122 & 3.2 & 36 & 0.32 & 9.90 \\
\hline 6 & 122 & 3.2 & 36 & 0.31 & 10.23 \\
\hline 7 & 131.2 & 3.2 & 38 & 0.31 & 10.32 \\
\hline 8 & 122.2 & 3.9 & 39 & 0.26 & 14.76 \\
\hline 9 & 109.1 & 5 & 39 & 0.28 & 17.69 \\
\hline 10 & 109.1 & 3.8 & 35 & 0.35 & 10.96 \\
\hline 11 & 109.1 & 3.8 & 35 & 0.35 & 10.93 \\
\hline 12 & 135.1 & 2.2 & 35 & 0.29 & 7.47 \\
\hline 13 & 122 & 3.2 & 35 & 0.32 & 9.85 \\
\hline 14 & 135.1 & 2.2 & 35 & N.A A $^{d}$ & N.A A $^{d}$ \\
\hline 15 & 122 & 3.2 & 35 & N.A ${ }^{d}$ & N.A $A^{d}$ \\
\hline 16 & 109.1 & 3.7 & 37 & 0.25 & 14.51 \\
\hline 17 & 109.1 & 4.1 & 38 & 0.23 & 17.77 \\
\hline 18 & 109.1 & 4.1 & 38 & 0.27 & 15.12 \\
\hline 19 & 129.3 & 3.5 & 37 & 0.33 & 10.64 \\
\hline 20 & 129.3 & 3.5 & 37 & 0.34 & 10.39 \\
\hline $21 a$ & 127.6 & 4.2 & 41 & 0.28 & 14.84 \\
\hline $21 c$ & 127.6 & 4.6 & 41 & 0.23 & 20.34 \\
\hline $22 a$ & 129.3 & 3.9 & 38 & 0.33 & 11.73 \\
\hline $22 b$ & 129.3 & 4.2 & 38 & 0.30 & 13.87 \\
\hline $22 c$ & 129.3 & 4.3 & 38 & 0.28 & 15.35 \\
\hline 23 & 122.3 & 3.9 & 39 & 0.31 & 12.58 \\
\hline 24 & 122.3 & 3.9 & 39 & 0.28 & 13.80 \\
\hline 25 & 129.3 & 3.6 & 38 & 0.38 & 9.49 \\
\hline 26 & 129.3 & 3.6 & 38 & 0.31 & 11.58 \\
\hline 27 & 118.3 & 3.9 & 39 & 0.28 & 14.08 \\
\hline
\end{tabular}

atPSA = topological polar surface area $\left(\AA^{2}\right) ;{ }^{b} \mathrm{SLogP}=$ Wildman-Crippen calculated logP; ${ }^{c H A C}=$ heavy atom count; ${ }^{d}$ N.A. $=$ not applicable. ${ }^{e}$ Properties calculated using Forge 10.4.2 Revision: 24876, Copyright 2006-2015, Cresset BioMolecular Discovery, Ltd. Efficiency metrics calculations for kurkinorin (1) and kurkinorin derivatives based on activity in the G-protein cAMP assay. Ligand efficiency (LE) was calculated using the following equation: $L E=$ $-1.37 \times \log \left(\frac{\text { potency, } M}{\text { Heavy Atom Count }}\right)$. Lipophilic-adjusted ligand efficiency (LELP) was calculated using the following equation: $=\frac{\text { Ligand efficiency }}{\text { cLogP }}$. 


\section{Chemistry}

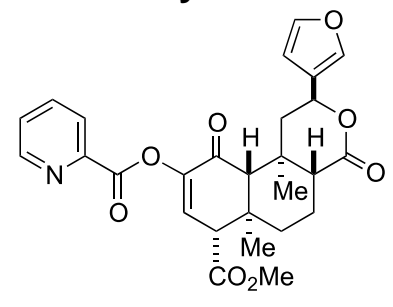

2S,4a $R, 6 \mathrm{a} R, 7 R, 10 \mathrm{a} R, 10 \mathrm{~b} R)$-2-(Furan-3-yl)-7-(methoxycarbonyl)6a,10b-dimethyl-4,10-dioxo-2,4,4a,5,6,6a,7,10,10a,10b-decahydro-1H-benzo[f] isochromen9-yl picolinate (4). ${ }^{1} \mathrm{H}$ NMR $\left(500 \mathrm{MHz}, \mathrm{CDCl}_{3}\right) \delta 8.82$ (ddd, $\left.J=0.87,1.69,4.72 \mathrm{~Hz}, 1 \mathrm{H}\right), 8.20$ (dt, $J=1.00,7.85 \mathrm{~Hz}, 1 \mathrm{H}), 7.90(\mathrm{td}, J=1.74,7.75 \mathrm{~Hz}, 1 \mathrm{H}), 7.55$ (ddd, $J=1.18,4.73,7.66 \mathrm{~Hz}, 1 \mathrm{H})$, $7.41(\mathrm{~m}, 1 \mathrm{H}), 7.39(\mathrm{t}, J=1.71 \mathrm{~Hz}, 1 \mathrm{H}), 6.75(\mathrm{~d}, J=2.17 \mathrm{~Hz}, 1 \mathrm{H}), 6.38(\mathrm{dd}, J=0.80,1.77 \mathrm{~Hz}, 1 \mathrm{H})$, $5.54(\mathrm{dd}, J=5.28,11.52 \mathrm{~Hz}, 1 \mathrm{H}), 3.79(\mathrm{~s}, 3 \mathrm{H}), 3.61(\mathrm{~d}, J=2.17 \mathrm{~Hz}, 1 \mathrm{H}), 3.07$ (dd, $J=5.35,13.67$ $\mathrm{Hz}, 1 \mathrm{H}), 2.49(\mathrm{~s}, 1 \mathrm{H}), 2.20(\mathrm{~m}, 2 \mathrm{H}), 2.14(\mathrm{~m}, 1 \mathrm{H}), 1.69(\mathrm{~m}, 3 \mathrm{H}), 1.38(\mathrm{~s}, 3 \mathrm{H}), 1.24(\mathrm{~s}, 3 \mathrm{H}) .{ }^{13} \mathrm{C}$ NMR $\left(126 \mathrm{MHz}, \mathrm{CDCl}_{3}\right) \delta 190.40,171.38,170.21,163.14,150.19,146.40,145.41,143.69$, 139.32, 137.24, 130.33, 127.70, 125.99, 125.45, 108.43, 72.07, 63.40, 56.51, 52.46, 51.34, 44.22, 43.72, 38.45, 35.85, 17.95, 16.81, 14.85. HRMS calculated for $\mathrm{C}_{27} \mathrm{H}_{27} \mathrm{NO}_{8}:[\mathrm{M}+\mathrm{Na}]^{+}: 516.1619$ (found), 516.1634 (calc). Melting point: $185-190^{\circ} \mathrm{C}$ (dec).

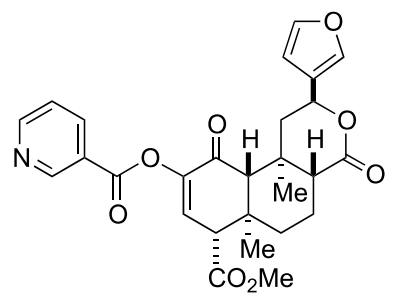

$(2 S, 4 \mathrm{a} R, 6 \mathrm{a} R, 7 R, 10 \mathrm{a} R, 10 \mathrm{~b} R)-2-($ Furan-3-yl)-7-(methoxycarbonyl)6a,10b-dimethyl-4,10-dioxo-2,4,4a,5,6,6a,7,10,10a,10b-decahydro-1H-benzo[ $f$ isochromen9-yl nicotinate (5). ${ }^{1} \mathrm{H}$ NMR $\left(500 \mathrm{MHz}, \mathrm{CDCl}_{3}\right) \delta 9.31(\mathrm{~s}, 1 \mathrm{H}), 8.85(\mathrm{~d}, J=3.16 \mathrm{~Hz}, 1 \mathrm{H}), 8.37$ (dt, $J=1.90,7.97 \mathrm{~Hz}, 1 \mathrm{H}), 7.45(\mathrm{dd}, J=4.89,7.94 \mathrm{~Hz}, 1 \mathrm{H}), 7.42(\mathrm{~m}, 1 \mathrm{H}), 7.40(\mathrm{t}, J=1.71 \mathrm{~Hz}, 1 \mathrm{H})$, $6.71(\mathrm{~d}, J=2.17 \mathrm{~Hz}, 1 \mathrm{H}), 6.39$ (dd, $J=0.80,1.78 \mathrm{~Hz}, 1 \mathrm{H}), 5.55(\mathrm{dd}, J=5.28,11.52 \mathrm{~Hz}, 1 \mathrm{H}), 3.81$ (s, 3H), $3.61(\mathrm{~d}, J=2.17 \mathrm{~Hz}, 1 \mathrm{H}), 3.06(\mathrm{dd}, J=5.35,13.65 \mathrm{~Hz}, 1 \mathrm{H}), 2.48(\mathrm{~s}, 1 \mathrm{H}), 2.21(\mathrm{~m}, 2 \mathrm{H})$, $2.13(\mathrm{~m}, 1 \mathrm{H}), 1.70(\mathrm{~m}, 3 \mathrm{H}), 1.38(\mathrm{~s}, 3 \mathrm{H}), 1.25(\mathrm{~s}, 3 \mathrm{H}) .{ }^{13} \mathrm{C}$ NMR $\left(126 \mathrm{MHz}, \mathrm{CDCl}_{3}\right) \delta 190.65$, 171.29, 170.26, 163.28, 154.29, 151.45, 145.15, 143.71, 139.38, 137.67, 130.45, 125.41, 124.48, 123.50, 108.43, 72.02, 63.50, 56.46, 52.54, 51.35, 44.26, 43.83, 38.44, 35.88, 17.94, 16.83, 14.86. HRMS calculated for $\mathrm{C}_{27} \mathrm{H}_{27} \mathrm{NO}_{8}:[\mathrm{M}-\mathrm{H}]=492.1640$ (found), 492.1664 (calc). Melting point: $109-112^{\circ} \mathrm{C}$.

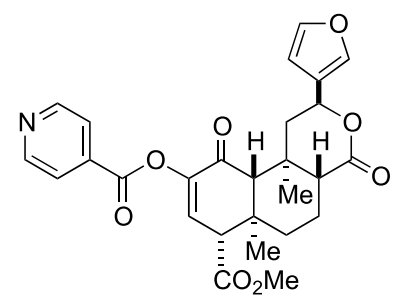

$(2 S, 4 \mathrm{a} R, 6 \mathrm{a} R, 7 R, 10 \mathrm{a} R, 10 \mathrm{~b} R)-2-($ Furan-3-yl)-7-(methoxycarbonyl)6a,10b-dimethyl-4,10-dioxo-2,4,4a,5,6,6a,7,10,10a,10b-decahydro-1 $\mathrm{H}$-benzo[f]isochromen9-yl isonicotinate (6). ${ }^{1} \mathrm{H}$ NMR $\left(500 \mathrm{MHz} \mathrm{CDCl}_{3}\right) \delta 8.84$ (dd, $\left.J=1.39,4.55 \mathrm{~Hz}, 2 \mathrm{H}\right), 7.91$ (dd, $J$ $=1.61,4.42 \mathrm{~Hz}, 2 \mathrm{H}), 7.42(\mathrm{~m}, 1 \mathrm{H}), 7.40(\mathrm{t}, J=1.69 \mathrm{~Hz}, 1 \mathrm{H}), 6.71(\mathrm{~d}, J=2.15 \mathrm{~Hz}, 1 \mathrm{H}), 6.39$ (dd, $J=0.73,1.69 \mathrm{~Hz}, 1 \mathrm{H}), 5.55(\mathrm{dd}, J=5.27,11.53 \mathrm{~Hz}, 1 \mathrm{H}), 3.81(\mathrm{~s}, 3 \mathrm{H}), 3.61(\mathrm{~d}, J=2.14 \mathrm{~Hz}, 1 \mathrm{H})$, $3.05(\mathrm{dd}, J=5.35,13.65 \mathrm{~Hz}, 1 \mathrm{H}), 2.47(\mathrm{~s}, 1 \mathrm{H}), 2.21(\mathrm{~m}, 2 \mathrm{H}), 2.14(\mathrm{~m}, 1 \mathrm{H}), 1.69(\mathrm{~m}, 3 \mathrm{H}), 1.38(\mathrm{~s}$, $3 \mathrm{H}), 1.24(\mathrm{~s}, 3 \mathrm{H}) .{ }^{13} \mathrm{C}$ NMR $\left(126 \mathrm{MHz}, \mathrm{CDCl}_{3}\right) \delta 190.47,171.24,170.22,163.18,150.87,145.12$, 143.72 , 139.38, 135.59, 130.49, 125.40, 123.19, 108.42, 72.00, 63.52, 56.44, 52.56, 51.34, 44.28, 
43.83, 38.45, 35.88, 17.94, 16.82, 14.85. HRMS calculated for $\mathrm{C}_{27} \mathrm{H}_{27} \mathrm{NO}_{8}:[\mathrm{M}+\mathrm{Na}]^{+}: 492.1624$ (found), 492.1664 (calc). Melting point: $112-114^{\circ} \mathrm{C}$.

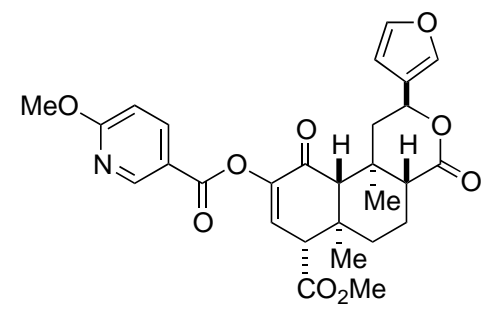

$(2 S, 4 \mathrm{a} R, 6 \mathrm{a} R, 7 R, 10 \mathrm{a} R, 10 \mathrm{~b} R)-2-($ Furan-3-yl)-7(methoxycarbonyl)-6a,10b-dimethyl-4,10-dioxo-2,4,4a,5,6,6a,7,10,10a,10b-decahydro-1 $H$ benzo[f]isochromen-9-yl 6-methoxynicotinate (7). ${ }^{1} \mathrm{H}$ NMR $\left(500 \mathrm{MHz}, \mathrm{CDCl}_{3}\right) \delta 8.91$ (dd, $J=$ $0.64,2.40 \mathrm{~Hz}, 1 \mathrm{H}), 8.19(\mathrm{dd}, J=2.41,8.74 \mathrm{~Hz}, 1 \mathrm{H}), 7.42(\mathrm{dt}, J=0.81,1.58 \mathrm{~Hz}, 1 \mathrm{H}), 7.39(\mathrm{t}, J=$ $1.71 \mathrm{~Hz}, 1 \mathrm{H}), 6.80(\mathrm{dd}, J=0.65,8.74 \mathrm{~Hz}, 1 \mathrm{H}), 6.66(\mathrm{~d}, J=2.18 \mathrm{~Hz}, 1 \mathrm{H}), 6.39(\mathrm{dd}, J=0.81,1.80$ $\mathrm{Hz}, 1 \mathrm{H}), 5.55(\mathrm{dd}, J=5.25,11.51 \mathrm{~Hz}, 1 \mathrm{H}), 4.02(\mathrm{~s}, 3 \mathrm{H}), 3.80(\mathrm{~s}, 3 \mathrm{H}), 3.59(\mathrm{~d}, J=2.18 \mathrm{~Hz}, 1 \mathrm{H})$, $3.06(\mathrm{dd}, J=5.34,13.66 \mathrm{~Hz}, 1 \mathrm{H}), 2.46(\mathrm{~s}, 1 \mathrm{H}), 2.20(\mathrm{~m}, 2 \mathrm{H}), 2.12(\mathrm{~m}, 1 \mathrm{H}), 1.68(\mathrm{~m}, 3 \mathrm{H}), 1.38(\mathrm{~s}$, $3 \mathrm{H}), 1.24(\mathrm{~s}, 3 \mathrm{H}) .{ }^{13} \mathrm{C}$ NMR $\left(126 \mathrm{MHz}, \mathrm{CDCl}_{3}\right) \delta 190.91,171.36,170.36,167.42,163.42,151.05$, 145.26, 143.69, 139.93, 139.38, 130.20, 125.43, 117.84, 110.99, 108.45, 72.04, 63.48, 56.49, 54.22, 52.49, 51.36, 44.21, 43.82, 38.44, 35.86, 17.95, 16.83, 14.86. HRMS: $[\mathrm{M}+\mathrm{Na}]^{+}: 546.1735$ (found), 546.1740 (calc). Melting point: $192-195^{\circ} \mathrm{C}$ (dec.).

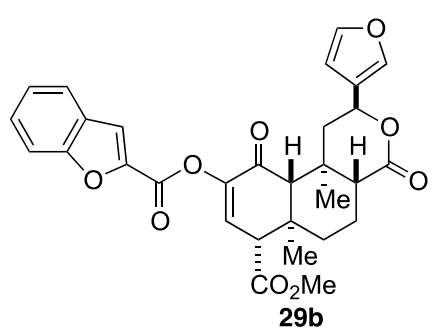

$(2 S, 4 \mathrm{a} R, 6 \mathrm{a} R, 7 R, 10 \mathrm{a} R, 10 \mathrm{~b} R)-$ Methyl

9-((benzofuran-2-

carbonyl)oxy)-2-(furan-3-yl)-6a,10b-dimethyl-4,10-dioxo-2,4,4a,5,6,6a,7,10,10a,10bdecahydro-1H-benzo[f]isochromene-7-carboxylate (8).

${ }^{1} \mathrm{H}$ NMR $\left(500 \mathrm{MHz}, \mathrm{CDCl}_{3}\right) \delta 7.72(\mathrm{~d}, J=7.87 \mathrm{~Hz}, 1 \mathrm{H}), 7.70(\mathrm{~d}, J=0.73 \mathrm{~Hz}, 1 \mathrm{H}), 7.61(\mathrm{~d}, J=8.43$ $\mathrm{Hz}, 1 \mathrm{H}), 7.50(\mathrm{~m}, 1 \mathrm{H}), 7.41(\mathrm{~m}, 1 \mathrm{H}), 7.39(\mathrm{t}, J=1.67 \mathrm{~Hz}, 1 \mathrm{H}), 7.34(\mathrm{t}, J=7.53 \mathrm{~Hz}, 1 \mathrm{H}), 6.73(\mathrm{~d}, J$ $=2.14 \mathrm{~Hz}, 1 \mathrm{H}), 6.39(\mathrm{~d}, J=0.96 \mathrm{~Hz}, 1 \mathrm{H}), 5.55(\mathrm{dd}, J=5.24,11.45 \mathrm{~Hz}, 1 \mathrm{H}), 3.80(\mathrm{~s}, 3 \mathrm{H}), 3.61(\mathrm{~d}$, $J=2.14 \mathrm{~Hz}, 1 \mathrm{H}), 3.06(\mathrm{dd}, J=5.34,13.67 \mathrm{~Hz}, 1 \mathrm{H}), 2.49(\mathrm{~s}, 1 \mathrm{H}), 2.20(\mathrm{~m}, 2 \mathrm{H}), 2.14(\mathrm{~m}, 1 \mathrm{H}), 1.70$ (m, 3H), 1.38 (s, 3H), 1.24 (s, 3H). ${ }^{13} \mathrm{C}$ NMR (126 MHz, $\left.\mathrm{CDCl}_{3}\right) \delta$ 190.54, 171.35, 170.24, 157.05, $156.17,144.73,143.69,143.64,139.36,130.48,128.41,126.76,125.41,124.11,123.13,116.22$, 112.49, 108.44, 72.06, 63.40, 56.46, 52.51, 51.31, 44.23, 43.71, 38.45, 35.84, 17.95, 16.82, 14.85. HRMS calculated for $\mathrm{C}_{30} \mathrm{H}_{28} \mathrm{O}_{9}:[\mathrm{M}+\mathrm{Na}]^{+}: 555.1611$ (found), 555.1631 (calc). Melting point: $126-130^{\circ} \mathrm{C}$.

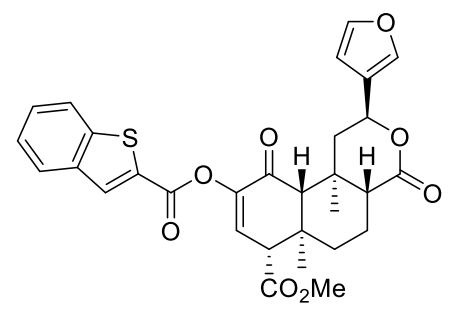

Methyl (2S,4aR,6aR,7R,10aR,10bR)-9-((benzo[b]thiophene-2carbonyl)oxy)-2-(furan-3-yl)-6a,10b-dimethyl-4,10-dioxo-1,4,4a,5,6,6a,7,10,10a,10bdecahydro-2H-benzo[f]isochromene-7-carboxylate (9). ${ }^{1} \mathrm{H}$ NMR $\left(500 \mathrm{MHz}, \mathrm{CDCl}_{3}\right) 8.20$ (d, J $=0.7 \mathrm{~Hz}, 1 \mathrm{H}), 7.90(\mathrm{td}, \mathrm{J}=7.5,1.0 \mathrm{~Hz}, 2 \mathrm{H}), 7.50$ (ddd, J = 8.2, 7.0, 1.3 Hz, 1H), 7.47 - $7.43(\mathrm{~m}$, 
$1 \mathrm{H}), 7.43-7.37(\mathrm{~m}, 2 \mathrm{H}), 6.72(\mathrm{~d}, \mathrm{~J}=2.2 \mathrm{~Hz}, 1 \mathrm{H}), 6.39(\mathrm{dd}, \mathrm{J}=1.9,0.9 \mathrm{~Hz}, 1 \mathrm{H}), 5.55(\mathrm{dd}, \mathrm{J}=$ 11.5, $5.2 \mathrm{~Hz}, 1 \mathrm{H}), 3.81(\mathrm{~s}, 3 \mathrm{H}), 3.60(\mathrm{~d}, \mathrm{~J}=2.2 \mathrm{~Hz}, 1 \mathrm{H}), 3.08(\mathrm{dd}, \mathrm{J}=13.7,5.3 \mathrm{~Hz}, 1 \mathrm{H}), 2.47$ (s, $1 \mathrm{H}), 2.24-2.17(\mathrm{~m}, 2 \mathrm{H}), 2.15-2.10(\mathrm{~m}, 1 \mathrm{H}), 1.77-1.72(\mathrm{~m}, 1 \mathrm{H}), 1.71-1.64(\mathrm{~m}, 2 \mathrm{H}), 1.39(\mathrm{~s}$, $3 \mathrm{H}), 1.25$ (s, 3H). ${ }^{13} \mathrm{C}$ NMR $\left(126 \mathrm{MHz}, \mathrm{CDCl}_{3}\right) \delta 190.65,171.36,170.28,160.54,145.06,143.67$, 142.79, 139.36, 138.53, 132.51, 131.11, 130.37, 127.54, 125.85, 125.40, 125.16, 122.81, 108.44, 72.06, 63.47, 56.46, 52.51, 51.35, 44.22, 43.80, 38.44, 35.86, 17.94, 16.83, 14.85. HRMS calculated for $\mathrm{C}_{30} \mathrm{H}_{28} \mathrm{O}_{8} \mathrm{~S}[\mathrm{M}+\mathrm{Na}]^{+}: 549.1561$ (found); 549.1578 (calcd). Melting point: $114-116{ }^{\circ} \mathrm{C}$.

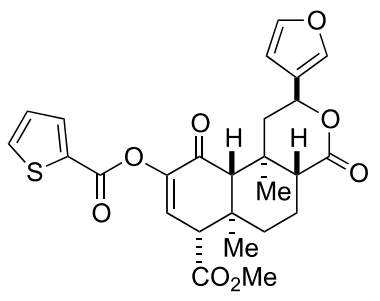

$(2 S, 4 \mathrm{a} R, 6 \mathrm{a} R, 7 R, 10 \mathrm{a} R, 10 \mathrm{~b} R)$-Methyl 2-(furan-3-yl)-6a,10b-dimethyl4,10-dioxo-9-((thiophene-2-carbonyl)oxy)-2,4,4a,5,6,6a,7,10,10a,10b-decahydro-1 Hbenzo[f]isochromene-7-carboxylate (10). ${ }^{1} \mathrm{H}$ NMR $\left(500 \mathrm{MHz}, \mathrm{CDCl}_{3}\right) \delta 7.92$ (dd, $J=1.26,3.79$ $\mathrm{Hz}, 1 \mathrm{H}), 7.67(\mathrm{dd}, J=1.25,4.96 \mathrm{~Hz}, 1 \mathrm{H}), 7.41(\mathrm{dt}, J=0.81,1.58 \mathrm{~Hz}, 1 \mathrm{H}), 7.39(\mathrm{t}, J=1.71 \mathrm{~Hz}$, $1 \mathrm{H}), 7.16(\mathrm{dd}, J=3.81,4.97 \mathrm{~Hz}, 1 \mathrm{H}), 6.67(\mathrm{~d}, J=2.18 \mathrm{~Hz}, 1 \mathrm{H}), 6.39(\mathrm{dd}, J=0.79,1.79 \mathrm{~Hz}, 1 \mathrm{H})$, 5.54 (dd, $J=5.25,11.52 \mathrm{~Hz}, 1 \mathrm{H}$ ), $3.80(\mathrm{~s}, 3 \mathrm{H}), 3.59$ (d, $J=2.17 \mathrm{~Hz}, 1 \mathrm{H}$ ), 3.07 (dd, $J=5.34,13.66$ $\mathrm{Hz}, 1 \mathrm{H}), 2.46(\mathrm{~s}, 1 \mathrm{H}), 2.20$ (ddd, $J=2.73,6.71,11.74 \mathrm{~Hz}, 2 \mathrm{H}), 2.12(\mathrm{~m}, 1 \mathrm{H}), 1.68(\mathrm{~m}, 3 \mathrm{H}), 1.38$ (s, 3H), 1.23 (s, 3H). ${ }^{13} \mathrm{C}$ NMR $\left(126 \mathrm{MHz} \mathrm{CDCl}_{3}\right) \delta$ 190.83, 171.37, 170.33, 159.89, 145.06, 143.68, 139.37, 135.24, 134.08, 131.34, 130.25, 128.14, 125.43, 108.44, 72.07, 63.46, 56.48, 52.48, 51.37, 44.21, 43.82, 38.45, 35.85, 17.95, 16.82, 14.86. HRMS calculated for $\mathrm{C}_{26} \mathrm{H}_{26} \mathrm{O}_{8} \mathrm{~S}$ : $[\mathrm{M}-\mathrm{H}]: 521.1254$ (found), 521.1246 (calc). Melting point: $150-152{ }^{\circ} \mathrm{C}$ (dec).

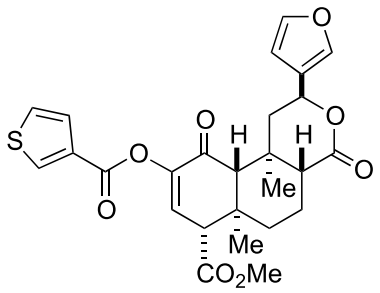

(2S,4a $R, 6 \mathrm{a} R, 7 R, 10 \mathrm{a} R, 10 \mathrm{~b} R)$-Methyl 2-(furan-3-yl)-6a,10b-dimethyl4,10-dioxo-9-((thiophene-3-carbonyl)oxy)-2,4,4a,5,6,6a,7,10,10a,10b-decahydro-1 Hbenzo[f]isochromene-7-carboxylate (11). ${ }^{1} \mathrm{H}$ NMR $\left(500 \mathrm{MHz}, \mathrm{CDCl}_{3}\right) \delta 8.26$ (dd, $J=1.20,3.03$ $\mathrm{Hz}, 1 \mathrm{H}), 7.58(\mathrm{dd}, J=1.20,5.10 \mathrm{~Hz}, 1 \mathrm{H}), 7.41(\mathrm{dd}, J=0.76,1.52 \mathrm{~Hz}, 1 \mathrm{H}), 7.39$ (t, $J=1.71 \mathrm{~Hz}$, $1 \mathrm{H}), 7.36(\mathrm{dd}, J=3.05,5.10 \mathrm{~Hz}, 1 \mathrm{H}), 6.64(\mathrm{~d}, J=2.17 \mathrm{~Hz}, 1 \mathrm{H}), 6.39$ (dd, $J=0.80,1.78 \mathrm{~Hz}, 1 \mathrm{H})$, $5.54(\mathrm{dd}, J=5.25,11.52 \mathrm{~Hz}, 1 \mathrm{H}), 3.80(\mathrm{~s}, 3 \mathrm{H}), 3.59(\mathrm{~d}, J=2.16 \mathrm{~Hz}, 1 \mathrm{H}), 3.07$ (dd, $J=5.33,13.66$ $\mathrm{Hz}, 1 \mathrm{H}), 2.47(\mathrm{~s}, 1 \mathrm{H}), 2.20(\mathrm{~m}, 2 \mathrm{H}), 2.12(\mathrm{~m}, 1 \mathrm{H}), 1.70(\mathrm{~m}, 3 \mathrm{H}), 1.38(\mathrm{~s}, 3 \mathrm{H}), 1.23(\mathrm{~s}, 3 \mathrm{H}) .{ }^{13} \mathrm{C}$ NMR $\left(126 \mathrm{MHz}_{2} \mathrm{CDCl}_{3}\right) \delta 190.98,171.39,170.39,160.29,145.19,143.67,139.37,134.68$, $131.42,130.05,128.13,126.56,125.43,108.45,72.06,63.43,56.48,52.47,51.36,44.20,43.80$, 38.45, 35.84, 17.95, 16.81, 14.86. HRMS calculated for $\mathrm{C}_{26} \mathrm{H}_{26} \mathrm{O}{ }_{8} \mathrm{~S}$ : [M+Na] $]^{+}: 521.1231$ (found), 521.1246 (calc). Melting point: $175-176{ }^{\circ} \mathrm{C}$ (dec). 


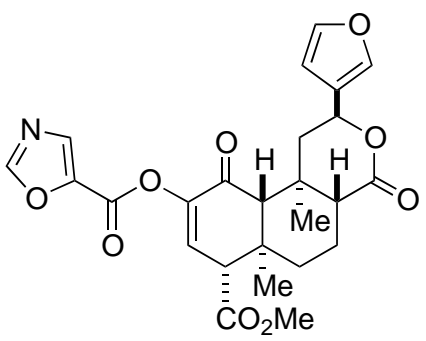

$(2 S, 4 \mathrm{a} R, 6 \mathrm{a} R, 7 R, 10 \mathrm{a} R, 10 \mathrm{~b} R)$-2-(Furan-3-yl)-7-(methoxycarbonyl)6a,10b-dimethyl-4,10-dioxo-2,4,4a,5,6,6a,7,10,10a,10b-decahydro-1 $H$-benzo[ $f$ isochromen9-yl oxazole-5-carboxylate (12). ${ }^{1} \mathrm{H}$ NMR $\left(500 \mathrm{MHz}, \mathrm{CDCl}_{3}\right) \delta 8.09$ (s, 1H), $7.94(\mathrm{~s}, 1 \mathrm{H}), 7.42$ $(\mathrm{dt}, J=0.82,1.60 \mathrm{~Hz}, 1 \mathrm{H}), 7.40(\mathrm{t}, J=1.71 \mathrm{~Hz}, 1 \mathrm{H}), 6.72(\mathrm{~d}, J=2.17 \mathrm{~Hz}, 1 \mathrm{H}), 6.39(\mathrm{dd}, J=0.81$, $1.79 \mathrm{~Hz}, 1 \mathrm{H}), 5.55$ (dd, J=5.30, $11.50 \mathrm{~Hz}, 1 \mathrm{H}$ ), $3.80(\mathrm{~s}, 3 \mathrm{H}), 3.59$ (d, J = 2.17 Hz, $1 \mathrm{H}$ ), 3.05 (dd, $J=5.35,13.65 \mathrm{~Hz}, 1 \mathrm{H}), 2.46(\mathrm{~s}, 1 \mathrm{H}), 2.20(\mathrm{~m}, 2 \mathrm{H}), 2.13(\mathrm{~m}, 1 \mathrm{H}), 1.69(\mathrm{~m}, 3 \mathrm{H}), 1.37(\mathrm{~s}, 3 \mathrm{H}), 1.22$ (s, 3H). ${ }^{13} \mathrm{C}$ NMR $\left(126 \mathrm{MHz}, \mathrm{CDCl}_{3}\right) \delta 190.37,171.24,170.11,154.90,154.13,144.35,143.73$, 141.26, 139.36, 135.43, 130.85, 125.39, 108.41, 72.01, 63.47, 56.41, 52.57, 51.30, 44.28, 43.77, 38.43, 35.86, 17.92, 16.80, 14.84. HRMS: [M+Na] ${ }^{+}: 506.1426$ (found), 506.1427 (calc). Melting point: $213-215^{\circ} \mathrm{C}$ (dec.).

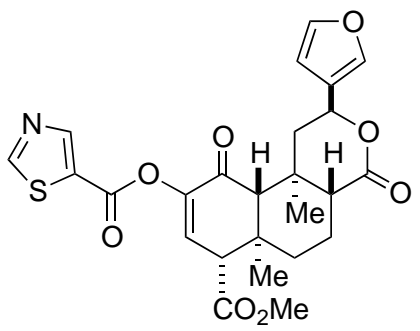

$(2 S, 4 \mathrm{a} R, 6 \mathrm{a} R, 7 R, 10 \mathrm{a} R, 10 \mathrm{~b} R)-2-($ Furan-3-yl)-7-(methoxycarbonyl)6a,10b-dimethyl-4,10-dioxo-2,4,4a,5,6,6a,7,10,10a,10b-decahydro-1 $H$-benzo[ $f$ ] isochromen9-yl thiazole-5-carboxylate (13). ${ }^{1} \mathrm{H}$ NMR $\left(500 \mathrm{MHz}, \mathrm{CDCl}_{3}\right) \delta 9.05$ (s, 1H), 8.63 (d, $J=4.73 \mathrm{~Hz}$, $1 \mathrm{H}), 7.42(\mathrm{~m}, 1 \mathrm{H}), 7.39(\mathrm{t}, J=1.68 \mathrm{~Hz}, 1 \mathrm{H}), 6.71(\mathrm{~d}, J=2.17 \mathrm{~Hz}, 1 \mathrm{H}), 6.39(\mathrm{dd}, J=0.79,1.79 \mathrm{~Hz}$, $1 \mathrm{H}), 5.55(\mathrm{dd}, J=5.43,11.47 \mathrm{~Hz}, 1 \mathrm{H}), 3.81(\mathrm{~s}, 2 \mathrm{H}), 3.60(\mathrm{~d}, J=2.16 \mathrm{~Hz}, 1 \mathrm{H}), 3.05(\mathrm{dd}, J=5.30$, $13.61 \mathrm{~Hz}, 1 \mathrm{H}), 2.46(\mathrm{~s}, 1 \mathrm{H}), 2.20(\mathrm{~m}, 2 \mathrm{H}), 2.12(\mathrm{~m}, 1 \mathrm{H}), 1.71(\mathrm{~m}, 4 \mathrm{H}), 1.37(\mathrm{~s}, 3 \mathrm{H}), 1.23(\mathrm{~s}, 3 \mathrm{H})$. ${ }^{13} \mathrm{C}$ NMR $\left(126 \mathrm{MHz}, \mathrm{CDCl}_{3}\right) \delta 190.55,171.29,170.56,170.19,159.33,158.86,150.53,144.76$, 143.71, 139.38, 130.68, 125.39, 108.43, 72.01, 63.44, 56.40, 52.55, 51.30, 44.26, 43.79, 38.41, 35.86, 17.92, 16.81, 14.84. HRMS: [M+Na] $]^{+} 522.1181$ (found), 522.1199 (calc). Melting point: $211-212^{\circ} \mathrm{C}(\mathrm{dec})$.

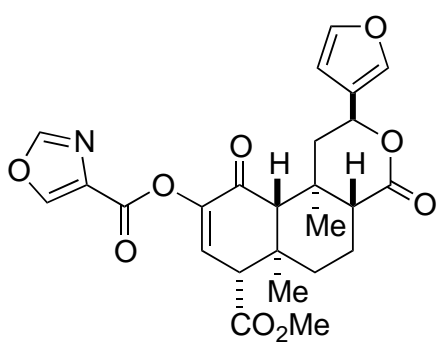

$(2 S, 4 \mathrm{a} R, 6 \mathrm{a} R, 7 R, 10 \mathrm{a} R, 10 \mathrm{~b} R)-2-($ Furan-3-yl)-7-(methoxycarbonyl)6a,10b-dimethyl-4,10-dioxo-2,4,4a,5,6,6a,7,10,10a,10b-decahydro-1 $H$-benzo[ $f$ ] isochromen9-yl oxazole-4-carboxylate (14). ${ }^{1} \mathrm{H}$ NMR (500 MHz, $\left.\mathrm{CDCl}_{3}\right) \delta 8.41$ (d, J=0.96 Hz, 1H), 8.00 (d, $J=0.96 \mathrm{~Hz}, 1 \mathrm{H}), 7.41$ (dd, $J=0.83,1.50 \mathrm{~Hz}, 1 \mathrm{H}), 7.39$ (t, $J=1.70 \mathrm{~Hz}, 1 \mathrm{H}), 6.69$ (d, $J=2.17 \mathrm{~Hz}$, $1 \mathrm{H}), 6.39(\mathrm{dd}, J=0.77,1.76 \mathrm{~Hz}, 1 \mathrm{H}), 5.54(\mathrm{dd}, J=5.18,11.47 \mathrm{~Hz}, 1 \mathrm{H}), 3.79(\mathrm{~s}, 3 \mathrm{H}), 3.59(\mathrm{~d}, J=$ $2.16 \mathrm{~Hz}, 1 \mathrm{H}), 3.04(\mathrm{dd}, J=5.35,13.67 \mathrm{~Hz}, 1 \mathrm{H}), 2.48(\mathrm{~s}, 1 \mathrm{H}), 2.19(\mathrm{~m}, 2 \mathrm{H}), 2.12(\mathrm{~m}, 1 \mathrm{H}), 1.70(\mathrm{~m}$, $3 \mathrm{H}), 1.37(\mathrm{~s}, 3 \mathrm{H}), 1.21(\mathrm{~s}, 3 \mathrm{H}) .{ }^{13} \mathrm{C}$ NMR $\left(126 \mathrm{MHz} \mathrm{CDCl}_{3}\right) \delta 190.51,171.35,170.22,158.42$, 151.75, 145.50, 144.66, 143.69, 139.35, 131.69, 130.52, 125.42, 108.44, 72.04, 63.32, 56.40, 
52.49, 51.27, 44.21, 43.66, 38.42, 35.82, 17.93, 16.79, 14.84. HRMS: [M+Na] $]^{+}$506.1424 (found), 506.1427 (calc). Melting point: $209-213^{\circ} \mathrm{C}$ (dec.).

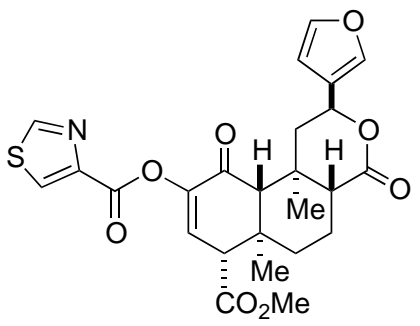

$(2 S, 4 \mathrm{a} R, 6 \mathrm{a} R, 7 R, 10 \mathrm{a} R, 10 \mathrm{~b} R)-2-($ Furan-3-yl)-7-(methoxycarbonyl)6a,10b-dimethyl-4,10-dioxo-2,4,4a,5,6,6a,7,10,10a,10b-decahydro-1H-benzo[ $f$ ] isochromen9-yl thiazole-4-carboxylate (15). ${ }^{1} \mathrm{H}$ NMR $\left(500 \mathrm{MHz}, \mathrm{CDCl}_{3}\right) \delta 8.90$ (d, $\left.J=2.08 \mathrm{~Hz}, 1 \mathrm{H}\right), 8.41$ (d, $J=2.07 \mathrm{~Hz}, 1 \mathrm{H}), 7.41(\mathrm{~m}, 1 \mathrm{H}), 7.39(\mathrm{t}, J=1.71 \mathrm{~Hz}, 1 \mathrm{H}), 6.72(\mathrm{~d}, J=2.17 \mathrm{~Hz}, 1 \mathrm{H}), 6.38(\mathrm{dd}, J=$ 0.79, $1.77 \mathrm{~Hz}, 1 \mathrm{H}), 5.54(\mathrm{dd}, J=5.30,11.51 \mathrm{~Hz}, 1 \mathrm{H}), 3.80(\mathrm{~s}, 3 \mathrm{H}), 3.60(\mathrm{~d}, J=2.17 \mathrm{~Hz}, 1 \mathrm{H}), 3.06$ (dd, $J=5.35,13.68 \mathrm{~Hz}, 1 \mathrm{H}), 2.49(\mathrm{~s}, 1 \mathrm{H}), 2.20$ (ddd, $J=2.69,6.54,9.86 \mathrm{~Hz}, 2 \mathrm{H}), 2.13(\mathrm{~m}, 1 \mathrm{H})$, $1.69(\mathrm{~m}, 3 \mathrm{H}), 1.37(\mathrm{~s}, 3 \mathrm{H}), 1.22(\mathrm{~s}, 3 \mathrm{H}) .{ }^{13} \mathrm{C} \mathrm{NMR}\left(126 \mathrm{MHz}, \mathrm{CDCl}_{3}\right) \delta$ 190.54, 171.36, 170.25, 158.78, 153.91, 146.10, 145.02, 143.70, 139.33, 130.34, 129.45, 125.43, 108.42, 72.07, 63.38, 56.47, 52.48, 51.33, 44.23, 43.71, 38.47, 35.84, 17.95, 16.81, 14.86. HRMS: [M+Na] ${ }^{+}: 522.1205$ (found), 522.1199 (calc). Melting point: $186^{\circ} \mathrm{C}$ (dec).

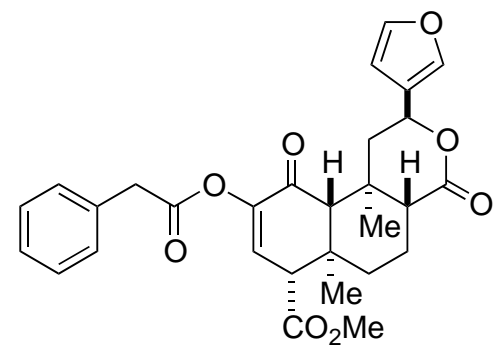

dimethyl-4,10-dioxo-9-(2-phenylacetoxy)-2,4,4a,5,6,6a,7,10,10a,10b-decahydro-1 $H$ benzo[f]isochromene-7-carboxylate (16). ${ }^{1} \mathrm{H}$ NMR $\left(500 \mathrm{MHz}, \mathrm{CDCl}_{3}\right) \delta 7.41(\mathrm{~d}, J=0.65 \mathrm{~Hz}$, $1 \mathrm{H}), 7.39(\mathrm{t}, J=1.65 \mathrm{~Hz}, 1 \mathrm{H}), 7.32(\mathrm{~m}, 5 \mathrm{H}), 6.50(\mathrm{~d}, J=2.15 \mathrm{~Hz}, 1 \mathrm{H}), 6.39(\mathrm{~d}, J=0.96 \mathrm{~Hz}, 1 \mathrm{H})$, 5.55 (dd, $J=5.25,11.53 \mathrm{~Hz}, 1 \mathrm{H}$ ), $3.83(\mathrm{~s}, 2 \mathrm{H}), 3.76(\mathrm{~s}, 3 \mathrm{H}), 3.50$ (d, J=2.12 Hz, 1H), 3.04 (dd, $J=5.32,13.63 \mathrm{~Hz}, 1 \mathrm{H}), 2.37(\mathrm{~s}, 1 \mathrm{H}), 2.16(\mathrm{~m}, 2 \mathrm{H}), 2.06(\mathrm{~m}, 1 \mathrm{H}), 1.65(\mathrm{~m}, 3 \mathrm{H}), 1.35(\mathrm{~s}, 3 \mathrm{H}), 1.16$ (s, 3H). ${ }^{13} \mathrm{C}$ NMR $\left(126 \mathrm{MHz}, \mathrm{CDCl}_{3}\right) \delta$ 190.72, 171.37, 170.30, 169.55, 145.26, 143.67, 139.43, 132.89, 129.89, 129.42, 128.70, 127.41, 125.40, 108.50, 71.99, 63.38, 56.34, 52.42, 51.29, 44.10, 43.69, 40.30, 38.35, 35.79, 17.90, 16.71, 14.81. HRMS: [M+Na] : 529.1826 (found), 529.1838 (calc). Melting point: $157-159^{\circ} \mathrm{C}$.

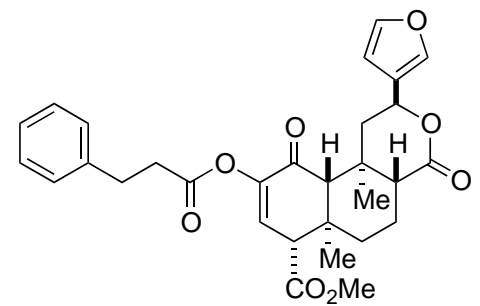

$(2 S, 4 a R, 6 a R, 7 R, 10 a R, 10 b R)-\quad$ Methyl 2-(furan-3-yl)-6a,10bdimethyl-4,10-dioxo-9-((3-phenylpropanoyl)oxy)-2,4,4a,5,6,6a,7,10,10a,10b-decahydro-1 $\mathrm{H}$ benzo[f]isochromene-7-carboxylate (17). ${ }^{1} \mathrm{H}$ NMR $\left(500 \mathrm{MHz}, \mathrm{CDCl}_{3}\right) \delta 7.42(\mathrm{~m}, 1 \mathrm{H}), 7.39(\mathrm{t}, \mathrm{J}$ $=1.70 \mathrm{~Hz}, 1 \mathrm{H}), 7.30(\mathrm{~m}, 2 \mathrm{H}), 7.22(\mathrm{~m}, 3 \mathrm{H}), 6.47(\mathrm{~d}, J=2.18 \mathrm{~Hz}, 1 \mathrm{H}), 6.40(\mathrm{dd}, J=0.78,1.78 \mathrm{~Hz}$, $1 \mathrm{H}), 5.56(\mathrm{dd}, J=5.25,11.49 \mathrm{~Hz}, 1 \mathrm{H}), 3.78(\mathrm{~s}, 3 \mathrm{H}), 3.51(\mathrm{~d}, J=2.17 \mathrm{~Hz}, 1 \mathrm{H}), 3.03(\mathrm{~m}, 3 \mathrm{H}), 2.83$ $(\mathrm{m}, 2 \mathrm{H}), 2.39(\mathrm{~s}, 1 \mathrm{H}), 2.17(\mathrm{~m}, 2 \mathrm{H}), 2.08(\mathrm{~m}, 1 \mathrm{H}), 1.66(\mathrm{~m}, 3 \mathrm{H}), 1.36(\mathrm{~s}, 3 \mathrm{H}), 1.17(\mathrm{~s}, 3 \mathrm{H}) .{ }^{13} \mathrm{C}$ 
NMR $\left(126 \mathrm{MHz}, \mathrm{CDCl}_{3}\right) \delta 190.95,171.37,170.78,170.32,145.17,143.69,139.96,139.41$, 129.84, 128.59, 128.28, 126.43, 125.42, 108.48, 72.01, 63.35, 56.36, 52.42, 51.30, 44.12, 43.72, 38.38, 35.80, 35.16, 30.69, 17.91, 16.73, 14.82. HRMS: [M+Na] : 543.1973 (found), 543.1995 (calc). Melting point: $162-165^{\circ} \mathrm{C}$.

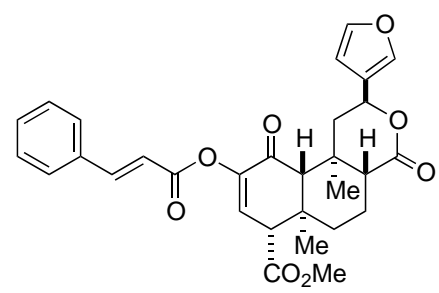

(2S,4a $R, 6 \mathrm{a} R, 7 R, 10 \mathrm{a} R, 10 \mathrm{~b} R)$ - Methyl 9-(cinnamoyloxy)-2-(furan-3yl)-6a,10b-dimethyl-4,10-dioxo-2,4,4a,5,6,6a,7,10,10a,10b-decahydro-1 $\mathrm{H}$ -

benzo[f]isochromene-7-carboxylate (18). ${ }^{1} \mathrm{H}$ NMR $\left(500 \mathrm{MHz}, \mathrm{CDCl}_{3}\right) \delta 7.82(\mathrm{~d}, J=16.01 \mathrm{~Hz}$, $1 \mathrm{H}), 7.55(\mathrm{~m}, 2 \mathrm{H}), 7.42(\mathrm{~m}, 4 \mathrm{H}), 7.39(\mathrm{t}, J=1.71 \mathrm{~Hz}, 1 \mathrm{H}), 6.61(\mathrm{~d}, J=2.19 \mathrm{~Hz}, 1 \mathrm{H}), 6.55(\mathrm{~d}, J=$ $15.99 \mathrm{~Hz}, 1 \mathrm{H}), 6.39$ (dd, $J=0.78,1.81 \mathrm{~Hz}, 1 \mathrm{H}), 5.56(\mathrm{dd}, J=5.26,11.49 \mathrm{~Hz}, 1 \mathrm{H}), 3.80(\mathrm{~s}, 3 \mathrm{H})$, $3.57(\mathrm{~d}, J=2.19 \mathrm{~Hz}, 1 \mathrm{H}), 3.08(\mathrm{dd}, J=5.34,13.68 \mathrm{~Hz}, 1 \mathrm{H}), 2.45(\mathrm{~s}, 1 \mathrm{H}), 2.20(\mathrm{~m}, 2 \mathrm{H}), 2.11(\mathrm{~m}$, $1 \mathrm{H}), 1.69$ (dd, $J=7.09,17.84 \mathrm{~Hz}, 3 \mathrm{H}), 1.38(\mathrm{~s}, 3 \mathrm{H}), 1.21(\mathrm{~s}, 3 \mathrm{H}) \cdot{ }^{13} \mathrm{C}$ NMR $\left(126 \mathrm{MHz}, \mathrm{CDCl}_{3}\right) \delta$ 191.07, 171.40, 170.40, 164.66, 147.64, 145.29, 143.68, 139.38, 133.92, 130.93, 129.83, 129.00, 128.39, 125.44, 115.75, 108.46, 72.07, 63.42, 56.47, 52.45, 51.38, 44.19, 43.78, 38.47, 35.85, 17.95, 16.79, 14.86. HRMS: [M+Na] ${ }^{+}: 541.1824$ (found), 541.1838 (calc). Melting point: $178{ }^{\circ} \mathrm{C}$ (dec.).

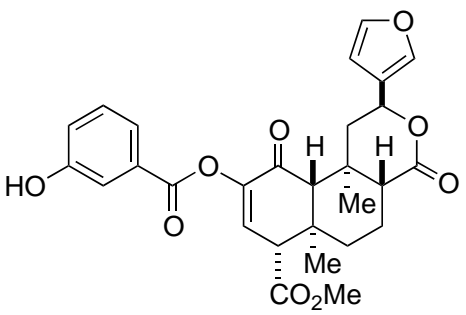

(2S,4a $R, 6 \mathrm{a} R, 7 R, 10 \mathrm{a} R, 10 \mathrm{~b} R)$ - Methyl 2-(furan-3-yl)-9-((3hydroxybenzoyl)oxy)-6a,10b-dimethyl-4,10-dioxo-2,4,4a,5,6,6a,7,10,10a,10b-decahydro1H-benzo[f]isochromene-7-carboxylate (19). ${ }^{1} \mathrm{H}$ NMR $\left(500 \mathrm{MHz}, \mathrm{CDCl}_{3}\right) \delta 7.66(\mathrm{~m}, 1 \mathrm{H}), 7.55$ $(\mathrm{dd}, J=1.56,2.49 \mathrm{~Hz}, 1 \mathrm{H}), 7.39(\mathrm{dt}, J=0.82,1.58 \mathrm{~Hz}, 1 \mathrm{H}), 7.38(\mathrm{t}, J=1.71 \mathrm{~Hz}, 1 \mathrm{H}), 7.33(\mathrm{t}, J=$ $7.94 \mathrm{~Hz}, 1 \mathrm{H}$ ), 7.10 (ddd, $J=0.86,2.61,8.13 \mathrm{~Hz}, 1 \mathrm{H}), 6.65$ (d, $J=2.16 \mathrm{~Hz}, 1 \mathrm{H}), 6.37$ (dd, $J=0.81$, $1.80 \mathrm{~Hz}, 1 \mathrm{H}), 6.02(\mathrm{~s}, 1 \mathrm{H}), 5.54(\mathrm{dd}, J=5.25,11.47 \mathrm{~Hz}, 1 \mathrm{H}), 3.80(\mathrm{~s}, 3 \mathrm{H}), 3.59(\mathrm{~d}, J=2.16 \mathrm{~Hz}$, $1 \mathrm{H}), 3.04(\mathrm{dd}, J=5.36,13.68 \mathrm{~Hz}, 1 \mathrm{H}), 2.47(\mathrm{~s}, 1 \mathrm{H}), 2.20(\mathrm{~m}, 2 \mathrm{H}), 2.11(\mathrm{~m}, 1 \mathrm{H}), 1.70(\mathrm{~m}, 3 \mathrm{H})$, $1.36(\mathrm{~s}, 3 \mathrm{H}), 1.23(\mathrm{~s}, 3 \mathrm{H}) .{ }^{13} \mathrm{C}$ NMR $\left(126 \mathrm{MHz} \mathrm{CDCl}_{3}\right) \delta 190.91,171.83,170.43,164.51,156.09$, 145.40, 143.67, 139.43, 130.13, 129.95, 129.49, 125.34, 122.52, 121.35, 116.82, 108.47, 72.19, $63.40,56.44,52.50,51.31,44.20,43.69,38.39,35.84,17.93,16.80,14.86$. HRMS: [M+Na] $]^{+}$ 531.1611 (found), 531.1631 (calc). Melting point: $124-126^{\circ} \mathrm{C}$.

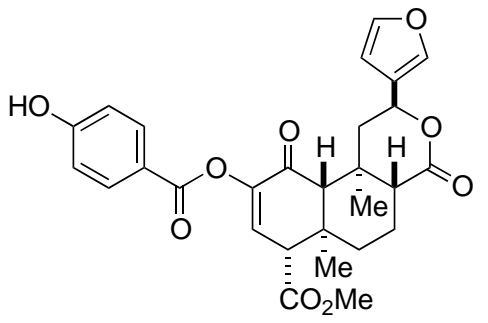

(2S,4a $R, 6 \mathrm{a} R, 7 R, 10 \mathrm{a} R, 10 \mathrm{~b} R)$ - Methyl 2-(furan-3-yl)-9-((4hydroxybenzoyl)oxy)-6a,10b-dimethyl-4,10-dioxo-2,4,4a,5,6,6a,7,10,10a,10b-decahydro1 H-benzo[f]isochromene-7-carboxylate (20). ${ }^{1} \mathrm{H}$ NMR $\left(500 \mathrm{MHz}, \mathrm{CDCl}_{3}\right) \delta 8.00(\mathrm{~d}, \mathrm{~J}=8.84 \mathrm{~Hz}$, 
2H), $7.40(\mathrm{dd}, J=0.75,1.50 \mathrm{~Hz}, 1 \mathrm{H}), 7.39(\mathrm{t}, J=1.70 \mathrm{~Hz}, 1 \mathrm{H}), 6.88(\mathrm{~d}, J=8.85 \mathrm{~Hz}, 2 \mathrm{H}), 6.64$ (d, $J=2.18 \mathrm{~Hz}, 1 \mathrm{H}), 6.38$ (dd, $J=0.79,1.77 \mathrm{~Hz}, 1 \mathrm{H}), 6.02(\mathrm{~s}, 1 \mathrm{H}), 5.54(\mathrm{dd}, J=5.25,11.52 \mathrm{~Hz}, 1 \mathrm{H})$, 3.80 (s, 3H), 3.59 (d, $J=2.18 \mathrm{~Hz}, 1 \mathrm{H}), 3.06$ (dd, $J=5.35,13.69 \mathrm{~Hz}, 1 \mathrm{H}), 2.47(\mathrm{~s}, 1 \mathrm{H}), 2.20(\mathrm{~m}$, $2 \mathrm{H}), 2.12(\mathrm{~m}, 1 \mathrm{H}), 1.69(\mathrm{~m}, 3 \mathrm{H}), 1.37(\mathrm{~s}, 3 \mathrm{H}), 1.23(\mathrm{~s}, 3 \mathrm{H}) .{ }^{13} \mathrm{C}$ NMR $\left(126 \mathrm{MHz}, \mathrm{CDCl}_{3}\right) \delta 191.22$, 171.66, 170.52, 164.34, 160.82, 145.48, 143.69, 139.40, 132.78, 129.99, 125.37, 120.56, 115.51, 108.45, 72.17, 63.42, 56.51, 52.49, 51.38, 44.21, 43.76, 38.47, 35.83, 17.94, 16.83, 14.88. HRMS: [M+Na] ${ }^{+}: 531.1625$ (found), 531.1631 (calc). Melting point: $136-139{ }^{\circ} \mathrm{C}$.

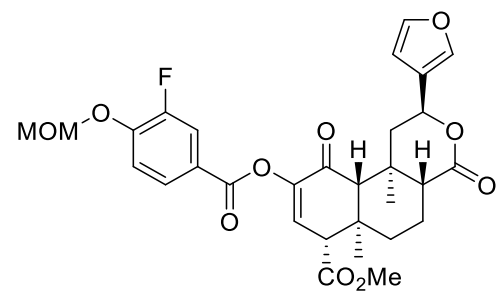

(methoxymethoxy)benzoyl)oxy)-2-(furan-3-yl)-6a,10b-dimethyl-4,10-dioxo-

1,4,4a,5,6,6a,7,10,10a,10b-decahydro-2H-benzo[f]isochromene-7-carboxylate (21a). ${ }^{1} \mathrm{H}$ $\operatorname{NMR}\left(500 \mathrm{MHz}, \mathrm{CDCl}_{3}\right) 7.95-7.71(\mathrm{~m}, 2 \mathrm{H}), 7.46-7.37(\mathrm{~m}, 2 \mathrm{H}), 6.65(\mathrm{~d}, \mathrm{~J}=2.2 \mathrm{~Hz}, 1 \mathrm{H}), 6.39$ (dd, J = 2.0, 0.9 Hz, 1H), $5.54(\mathrm{dt}, \mathrm{J}=11.6,5.8 \mathrm{~Hz}, 1 \mathrm{H}), 5.31(\mathrm{~s}, 2 \mathrm{H}), 3.80(\mathrm{~s}, 3 \mathrm{H}), 3.59(\mathrm{~d}, \mathrm{~J}=$ $2.3 \mathrm{~Hz}, 1 \mathrm{H}), 3.52(\mathrm{~d}, \mathrm{~J}=1.2 \mathrm{~Hz}, 3 \mathrm{H}), 3.06(\mathrm{dd}, \mathrm{J}=13.7,5.4 \mathrm{~Hz}, 1 \mathrm{H}), 2.46(\mathrm{~s}, 1 \mathrm{H}), 2.25-2.16(\mathrm{~m}$, $2 \mathrm{H}), 2.16-2.08(\mathrm{~m}, 1 \mathrm{H}), 1.79-1.63(\mathrm{~m}, 3 \mathrm{H}), 1.57-1.48(\mathrm{~m}, 1 \mathrm{H}), 1.37(\mathrm{~s}, 3 \mathrm{H}), 1.23(\mathrm{~s}, 3 \mathrm{H}) .{ }^{13} \mathrm{C}$ NMR (126 MHz, CDCl $)$ ) 190.86, 171.37, 170.36, 163.34, 152.20 (d, J = 247.7 Hz), 149.99 (d, J $=10.7 \mathrm{~Hz}$ ), 145.34, 143.68, 139.36, 130.05, 127.42, 125.41, 122.10, 118.22 (d, J = $20.5 \mathrm{~Hz}$ ), 116.44, 108.44, 95.08, 72.05, 63.46, 56.64, 56.48, 52.49, 51.37, 44.21, 43.80, 38.45, 35.85, 17.94, 16.82, 14.86. HRMS calculated for $\mathrm{C}_{30} \mathrm{H}_{31} \mathrm{O}_{10} \mathrm{~F}[\mathrm{M}+\mathrm{Na}]^{+}: 593.1788$ (found); 593.1799 (calcd). Melting point: $88-90^{\circ} \mathrm{C}$.

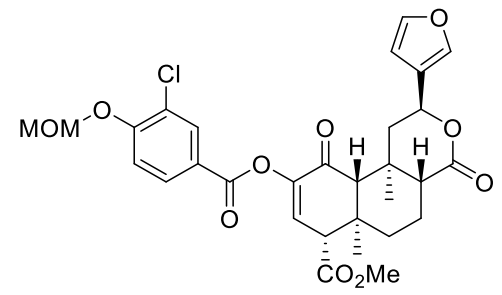

(methoxymethoxy)benzoyl)oxy)-2-(furan-3-yl)-6a,10b-dimethyl-4,10-dioxo-

Methyl (2S,4aR,6aR,7R,10aR,10bR)-9-((3-chloro-41,4,4a,5,6,6a,7,10,10a,10b-decahydro-2H-benzo[f]isochromene-7-carboxylate (21b). ${ }^{1} \mathrm{H}$ $\operatorname{NMR}\left(500 \mathrm{MHz}, \mathrm{CDCl}_{3}\right) 8.14(\mathrm{dd}, \mathrm{J}=3.4,2.2 \mathrm{~Hz}, 1 \mathrm{H}), 7.97(\mathrm{dd}, \mathrm{J}=8.7,2.1 \mathrm{~Hz}, 1 \mathrm{H}), 7.44-7.36$ $(\mathrm{m}, 2 \mathrm{H}), 7.24(\mathrm{~s}, 1 \mathrm{H}), 6.65(\mathrm{~d}, \mathrm{~J}=2.2 \mathrm{~Hz}, 1 \mathrm{H}), 6.39(\mathrm{dd}, \mathrm{J}=1.9,0.9 \mathrm{~Hz}, 1 \mathrm{H}), 5.54(\mathrm{dd}, \mathrm{J}=11.5$, $5.5 \mathrm{~Hz}, 1 \mathrm{H}), 5.34(\mathrm{~d}, \mathrm{~J}=1.5 \mathrm{~Hz}, 2 \mathrm{H}), 3.80(\mathrm{~s}, 3 \mathrm{H}), 3.59(\mathrm{~d}, \mathrm{~J}=2.2 \mathrm{~Hz}, 1 \mathrm{H}), 3.52(\mathrm{~d}, \mathrm{~J}=1.2 \mathrm{~Hz}$, 4H), $3.06(\mathrm{dd}, \mathrm{J}=13.7,5.4 \mathrm{~Hz}, 1 \mathrm{H}), 2.46(\mathrm{~s}, 1 \mathrm{H}), 2.24-2.15(\mathrm{~m}, 1 \mathrm{H}), 2.16-2.09(\mathrm{~m}, 1 \mathrm{H}), 1.78$ - $1.61(\mathrm{~m}, 3 \mathrm{H}), 1.37(\mathrm{~s}, 3 \mathrm{H}), 1.23(\mathrm{~s}, 3 \mathrm{H}) .{ }^{13} \mathrm{C} \mathrm{NMR}\left(126 \mathrm{MHz}, \mathrm{CDCl}_{3}\right) \delta$ 190.85, 171.37, 170.35, 163.26, 157.28, 145.34, 143.68, 139.36, 132.45, 130.45, 130.03, 125.40, 123.62, 122.33, 115.04, 108.44, 94.79, 72.05, 63.45, 56.63, 56.48, 52.49, 51.37, 44.21, 43.79, 38.46, 35.85, 17.94, 16.82, 14.86. HRMS calculated for $\mathrm{C}_{30} \mathrm{H}_{31} \mathrm{O}_{10} \mathrm{Cl}[\mathrm{M}+\mathrm{Na}]^{+}: 587.1754$ (found); 587.1679 (calcd). Melting point: $108-110^{\circ} \mathrm{C}$. 
<smiles>COc1ccc(C(=O)OC2=C[C@H](OC)[C@H]3CC[C@H]4C(=O)O[C@H](c5ccoc5)C[C@H]4[C@H]3C2=O)cc1Br</smiles>

(methoxymethoxy)benzoyl)oxy)-2-(furan-3-yl)-6a,10b-dimethyl-4,10-dioxo-

$\mathbf{1 , 4 , 4 a , 5 , 6 , 6 a , 7 , 1 0 , 1 0 a , 1 0 b - d e c a h y d r o - 2 H - b e n z o [ f ] i s o c h r o m e n e - 7 - c a r b o x y l a t e ~} \quad(21 \mathrm{c}) . \quad{ }^{1} \mathrm{H}$ NMR $\left(500 \mathrm{MHz} \mathrm{CDCl}_{3}\right) 8.31(\mathrm{dd}, \mathrm{J}=2.9,2.1 \mathrm{~Hz}, 1 \mathrm{H}), 8.06-7.95(\mathrm{~m}, 1 \mathrm{H}), 7.48-7.35(\mathrm{~m}, 2 \mathrm{H})$, $7.21(\mathrm{~d}, \mathrm{~J}=8.7 \mathrm{~Hz}, 1 \mathrm{H}), 6.65(\mathrm{~d}, \mathrm{~J}=2.2 \mathrm{~Hz}, 1 \mathrm{H}), 6.39(\mathrm{dd}, \mathrm{J}=2.0,0.9 \mathrm{~Hz}, 1 \mathrm{H}), 5.59-5.50(\mathrm{~m}$, $1 \mathrm{H}), 5.33(\mathrm{~d}, \mathrm{~J}=1.6 \mathrm{~Hz}, 2 \mathrm{H}), 3.80(\mathrm{~s}, 3 \mathrm{H}), 3.73(\mathrm{dd}, \mathrm{J}=5.3,3.3 \mathrm{~Hz}, 1 \mathrm{H}), 3.59(\mathrm{~d}, \mathrm{~J}=2.3 \mathrm{~Hz}, 1 \mathrm{H})$, $3.52(\mathrm{~d}, \mathrm{~J}=1.3 \mathrm{~Hz}, 3 \mathrm{H}), 3.06(\mathrm{dd}, \mathrm{J}=13.7,5.2 \mathrm{~Hz}, 1 \mathrm{H}), 2.47(\mathrm{~s}, 1 \mathrm{H}), 2.24-2.16(\mathrm{~m}, 2 \mathrm{H}), 1.82-$ $1.52(\mathrm{~m}, 3 \mathrm{H}), 1.37(\mathrm{~s}, 3 \mathrm{H}), 1.23(\mathrm{~s}, 3 \mathrm{H}) .{ }^{13} \mathrm{C} \mathrm{NMR}\left(126 \mathrm{MHz}, \mathrm{CDCl}_{3}\right) \delta$ 190.84, 171.37, 170.35, 163.11, 158.15, 145.33, 143.67, 139.36, 135.57, 131.17, 130.02, 125.40, 122.72, 114.78, 112.54, 108.44, 94.75, 72.05, 63.43, 56.63, 56.47, 52.48, 51.35, 44.21, 43.78, 38.45, 35.84, 17.94, 16.81, 14.85. HRMS calculated for $\mathrm{C}_{30} \mathrm{H}_{31} \mathrm{O}_{10} \mathrm{Br}[\mathrm{M}+\mathrm{Na}]^{+}: 631.1156$ (found); 631.1173 (calcd). Melting point: $84-87^{\circ} \mathrm{C}$.

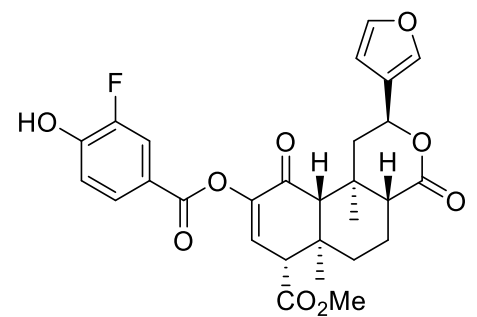

hydroxybenzoyl)oxy)-2-(furan-3-yl)-6a,10b-dimethyl-4,10-dioxo-1,4,4a,5,6,6a,7,10,10a,10bdecahydro-2H-benzo[f]isochromene-7-carboxylate (22a). ${ }^{1} \mathrm{H}$ NMR $\left(500 \mathrm{MHz}, \mathrm{CDCl}_{3}\right) 7.91$ $7.76(\mathrm{~m}, 2 \mathrm{H}), 7.47-7.32(\mathrm{~m}, 2 \mathrm{H}), 7.07(\mathrm{t}, \mathrm{J}=8.5 \mathrm{~Hz}, 1 \mathrm{H}), 6.65(\mathrm{~d}, \mathrm{~J}=2.2 \mathrm{~Hz}, 1 \mathrm{H}), 6.39(\mathrm{dd}, \mathrm{J}=$ 1.9, $0.9 \mathrm{~Hz}, 1 \mathrm{H}), 5.54(\mathrm{dd}, \mathrm{J}=11.5,5.3 \mathrm{~Hz}, 1 \mathrm{H}), 3.80(\mathrm{~s}, 3 \mathrm{H}), 3.59(\mathrm{~d}, \mathrm{~J}=2.2 \mathrm{~Hz}, 1 \mathrm{H}), 3.06$ (dd, $\mathrm{J}=13.6,5.3 \mathrm{~Hz}, 1 \mathrm{H}), 2.47(\mathrm{~s}, 1 \mathrm{H}), 2.25-2.16(\mathrm{~m}, 2 \mathrm{H}), 2.12(\mathrm{dt}, \mathrm{J}=12.9,2.6 \mathrm{~Hz}, 1 \mathrm{H}), 1.80-$ $1.56(\mathrm{~m}, 3 \mathrm{H}), 1.37(\mathrm{~s}, 3 \mathrm{H}), 1.23(\mathrm{~s}, 3 \mathrm{H}) .{ }^{13} \mathrm{C}$ NMR $\left(126 \mathrm{MHz}, \mathrm{CDCl}_{3}\right) \delta$ 190.96, 171.44, 170.37, 163.30, 150.40 (d, J = 239.3 Hz), 148.91 (d, J = 14.2 Hz), 145.31, 143.68, 139.36, 130.09, 128.03, 125.37, 120.96, 117.74 (d, J = 20.0 Hz), 117.28, 108.43, 72.07, 63.43, 56.47, 52.50, 51.36, 44.21, 43.78, 38.45, 35.84, 17.93, 16.81, 14.86. HRMS calculated for $\mathrm{C}_{28} \mathrm{H}_{27} \mathrm{O}_{9} \mathrm{~F}[\mathrm{M}+\mathrm{H}]^{+}: 527.173$ (found); 527.1711 (calcd). Melting point: $134-136^{\circ} \mathrm{C}$.

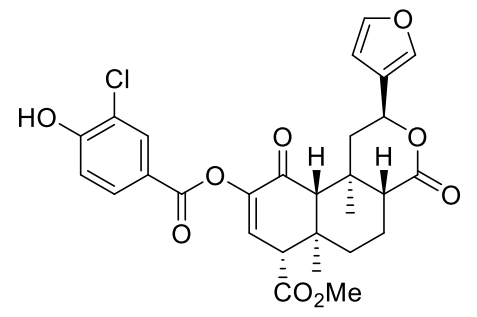

Methyl

(2S,4aR,6aR,7R,10aR,10bR)-9-((3-chloro-4hydroxybenzoyl)oxy)-2-(furan-3-yl)-6a,10b-dimethyl-4,10-dioxo-1,4,4a,5,6,6a,7,10,10a,10bdecahydro-2H-benzo[f]isochromene-7-carboxylate (22b). ${ }^{1} \mathrm{H}$ NMR $\left(500 \mathrm{MHz}, \mathrm{CDCl}_{3}\right) 8.12$ (d, $\mathrm{J}=2.0 \mathrm{~Hz}, 1 \mathrm{H}), 7.95(\mathrm{dd}, \mathrm{J}=8.4,1.9 \mathrm{~Hz}, 1 \mathrm{H}), 7.45-7.33(\mathrm{~m}, 2 \mathrm{H}), 7.09(\mathrm{~d}, \mathrm{~J}=8.6 \mathrm{~Hz}, 1 \mathrm{H}), 6.66$ $(\mathrm{d}, \mathrm{J}=2.1 \mathrm{~Hz}, 1 \mathrm{H}), 6.39(\mathrm{~s}, 1 \mathrm{H}), 6.26(\mathrm{~s}, 1 \mathrm{H}), 5.55(\mathrm{dd}, \mathrm{J}=11.6,5.3 \mathrm{~Hz}, 1 \mathrm{H}), 3.80(\mathrm{~s}, 3 \mathrm{H}), 3.60$ (d, J = 2.2 Hz, 1H), 3.49 (q, J = 7.0 Hz, OH), 3.05 (dd, J = 13.7, 5.3 Hz, 1H), $2.48(\mathrm{~s}, 1 \mathrm{H}), 2.24-$ $2.16(\mathrm{~m}, 2 \mathrm{H}), 2.16-2.02(\mathrm{~m}, 1 \mathrm{H}), 1.78-1.59(\mathrm{~m}, 3 \mathrm{H}), 1.37(\mathrm{~s}, 3 \mathrm{H}), 1.23(\mathrm{~s}, 3 \mathrm{H}) .{ }^{13} \mathrm{C}$ NMR $(126$ 
$\mathrm{MHz}, \mathrm{CDCl} 3) \delta 190.99,171.50,170.41,163.21,156.17,145.28,143.72,139.40,131.63,131.14$, 130.16, 125.34, 121.60, 120.26, 116.32, 108.46, 72.10, 63.36, 56.46, 52.57, 51.36, 44.25, 43.73, 38.45, 35.83, 17.94, 16.86, 14.87. HRMS calculated for $\mathrm{C}_{28} \mathrm{H}_{27} \mathrm{ClO}_{9}[\mathrm{M}+\mathrm{H}]^{+}: 543.1406$ (found); 543.1417 (calcd). Melting point: $127-129^{\circ} \mathrm{C}$ (decomp).

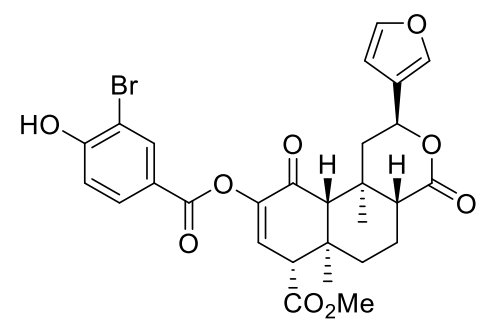

Methyl

(2S,4aR,6aR,7R,10aR,10bR)-9-((3-bromo-4hydroxybenzoyl)oxy)-2-(furan-3-yl)-6a,10b-dimethyl-4,10-dioxo-1,4,4a,5,6,6a,7,10,10a,10bdecahydro-2H-benzo[f]isochromene-7-carboxylate (22c). ${ }^{1} \mathrm{H} \mathrm{NMR}\left(500 \mathrm{MHz}, \mathrm{CDCl}_{3}\right) 8.27$ (d, $\mathrm{J}=2.1 \mathrm{~Hz}, 1 \mathrm{H}), 7.99(\mathrm{dd}, \mathrm{J}=8.6,2.1 \mathrm{~Hz}, 1 \mathrm{H}), 7.45-7.35(\mathrm{~m}, 2 \mathrm{H}), 7.09(\mathrm{~d}, \mathrm{~J}=8.5 \mathrm{~Hz}, 1 \mathrm{H}), 6.65$ $(\mathrm{d}, \mathrm{J}=2.2 \mathrm{~Hz}, 1 \mathrm{H}), 6.39$ (dd, J = 1.9, $0.9 \mathrm{~Hz}, 1 \mathrm{H}), 5.54(\mathrm{dd}, \mathrm{J}=11.5,5.3 \mathrm{~Hz}, 1 \mathrm{H}), 3.80(\mathrm{~s}, 3 \mathrm{H})$, 3.59 (d, J = 2.2 Hz, 1H), 3.06 (dd, J = 13.6, $5.3 \mathrm{~Hz}, 1 \mathrm{H}), 2.47(\mathrm{~s}, 1 \mathrm{H}), 2.20$ (td, J = 10.7, 9.6, 4.8 $\mathrm{Hz}, 2 \mathrm{H}), 2.15-2.09(\mathrm{~m}, 1 \mathrm{H}), 1.77-1.60(\mathrm{~m}, 3 \mathrm{H}), 1.37(\mathrm{~s}, 3 \mathrm{H}), 1.23(\mathrm{~s}, 3 \mathrm{H}) .{ }^{13} \mathrm{C} \mathrm{NMR}(126 \mathrm{MHz}$, $\left.\mathrm{CDCl}_{3}\right) \delta 190.91,171.35,170.33,162.98,157.00,145.30,143.67,139.36,134.63,131.84$, 130.06, 125.39, 122.04, 116.06, 110.32, 108.43, 72.04, 63.43, 56.47, 52.49, 51.36, 44.22, 43.79, 38.46, 35.84, 17.94, 16.81, 14.86. HRMS calculated for $\mathrm{C}_{28} \mathrm{H}_{27} \mathrm{O}_{9} \mathrm{Br}[\mathrm{M}+\mathrm{H}]^{+}: 587.0909$ (found); 587.0911 (calcd). Melting point: $144-147^{\circ} \mathrm{C}$.<smiles>CO[C@H]1C=C(OC(=O)c2ccc3ccoc3c2)C(=O)[C@@H]2[C@@H]1CC[C@H]1C(=O)O[C@@H](c3ccoc3)C[C@@H]21</smiles>

\section{Methyl}

(2S,4aR,6aR,7R,10aR,10bR)-9-((benzofuran-6carbonyl)oxy)-2-(furan-3-yl)-6a,10b-dimethyl-4,10-dioxo-1,4,4a,5,6,6a,7,10,10a,10b-

decahydro-2H-benzo[f]isochromene-7-carboxylate (23). ${ }^{1} \mathrm{H}$ NMR $\left(500 \mathrm{MHz}^{\mathrm{C}} \mathrm{CDCl}_{3}\right) 8.28(\mathrm{p}$, $\mathrm{J}=0.8 \mathrm{~Hz}, 1 \mathrm{H}), 8.01(\mathrm{dd}, \mathrm{J}=8.2,1.5 \mathrm{~Hz}, 1 \mathrm{H}), 7.80(\mathrm{~d}, \mathrm{~J}=2.2 \mathrm{~Hz}, 1 \mathrm{H}), 7.68(\mathrm{dd}, \mathrm{J}=8.2,0.6 \mathrm{~Hz}$, $1 \mathrm{H}), 7.49-7.36(\mathrm{~m}, 2 \mathrm{H}), 6.85(\mathrm{dd}, \mathrm{J}=2.2,1.0 \mathrm{~Hz}, 1 \mathrm{H}), 6.68(\mathrm{~d}, \mathrm{~J}=2.2 \mathrm{~Hz}, 1 \mathrm{H}), 6.39(\mathrm{dd}, \mathrm{J}=$ 1.9, $0.9 \mathrm{~Hz}, 1 \mathrm{H}), 5.60-5.46(\mathrm{~m}, 1 \mathrm{H}), 3.81(\mathrm{~s}, 3 \mathrm{H}), 3.61(\mathrm{~d}, \mathrm{~J}=2.2 \mathrm{~Hz}, 1 \mathrm{H}), 3.08$ (dd, J = 13.7, $5.3 \mathrm{~Hz}, 1 \mathrm{H}), 2.49(\mathrm{~s}, 1 \mathrm{H}), 2.26-2.17(\mathrm{~m}, 1 \mathrm{H}), 2.13(\mathrm{dt}, \mathrm{J}=13.2,2.7 \mathrm{~Hz}, 1 \mathrm{H}), 1.79-1.58(\mathrm{~m}, 2 \mathrm{H})$, $1.39(\mathrm{~s}, 3 \mathrm{H}), 1.26(\mathrm{~d}, \mathrm{~J}=2.2 \mathrm{~Hz}, 5 \mathrm{H}) .{ }^{13} \mathrm{C} \mathrm{NMR}\left(126 \mathrm{MHz}, \mathrm{CDCl}_{3}\right) \delta$ 190.96, 171.39, 170.40, 164.78, 154.30, 148.44, 145.52, 143.65, 139.36, 132.69, 129.94, 125.42, 124.74, 124.25, 121.15, 113.87, 108.45, 106.92, 72.07, 63.46, 56.51, 52.47, 51.38, 44.20, 43.80, 38.46, 35.85, 17.96, 16.84, 14.86. HRMS calculated for $\mathrm{C}_{30} \mathrm{H}_{28} \mathrm{O}_{9}[\mathrm{M}+\mathrm{H}]^{+}: 533.1819$ (found); 533.1806 (calcd). Melting point: $156-157^{\circ} \mathrm{C}$ (decomp). 


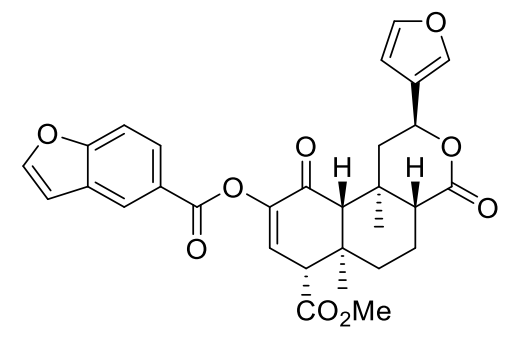

Methyl (2S,4aR,6aR,7R,10aR,10bR)-9-((benzofuran-5carbonyl)oxy)-2-(furan-3-yl)-6a,10b-dimethyl-4,10-dioxo-1,4,4a,5,6,6a,7,10,10a,10bdecahydro-2H-benzo[f]isochromene-7-carboxylate (24). ${ }^{1} \mathrm{H}$ NMR $\left(500 \mathrm{MHz}, \mathrm{CDCl}_{3}\right) 8.46-$ $8.40(\mathrm{~m}, 1 \mathrm{H}), 8.08(\mathrm{dd}, \mathrm{J}=8.7,1.8 \mathrm{~Hz}, 1 \mathrm{H}), 7.71(\mathrm{~d}, \mathrm{~J}=2.2 \mathrm{~Hz}, 1 \mathrm{H}), 7.57(\mathrm{dt}, \mathrm{J}=8.7,0.8 \mathrm{~Hz}$, $1 \mathrm{H}), 7.48-7.34(\mathrm{~m}, 2 \mathrm{H}), 6.86(\mathrm{dd}, \mathrm{J}=2.2,1.0 \mathrm{~Hz}, 1 \mathrm{H}), 6.68(\mathrm{~d}, \mathrm{~J}=2.2 \mathrm{~Hz}, 1 \mathrm{H}), 6.39(\mathrm{dd}, \mathrm{J}=$ 2.0, $0.9 \mathrm{~Hz}, 1 \mathrm{H}), 5.62-5.47(\mathrm{~m}, 1 \mathrm{H}), 3.80(\mathrm{~s}, 3 \mathrm{H}), 3.61(\mathrm{~d}, \mathrm{~J}=2.2 \mathrm{~Hz}, 1 \mathrm{H}), 3.08(\mathrm{dd}, \mathrm{J}=13.7$, $5.3 \mathrm{~Hz}, 1 \mathrm{H}), 2.49(\mathrm{~s}, 1 \mathrm{H}), 2.20(\mathrm{ddt}, \mathrm{J}=12.6,6.9,3.5 \mathrm{~Hz}, 2 \mathrm{H}), 2.12(\mathrm{dt}, \mathrm{J}=13.1,2.7 \mathrm{~Hz}, 1 \mathrm{H}), 1.79$ $-1.63(\mathrm{~m}, 3 \mathrm{H}), 1.39$ (s, 3H), 1.25 (s, 3H). ${ }^{13} \mathrm{C} \mathrm{NMR}\left(126 \mathrm{MHz}, \mathrm{CDCl}_{3}\right) \delta$ 191.04, 171.39, 170.42, 164.76, 157.96, 146.54, 145.52, 143.65, 139.36, 129.92, 127.66, 126.67, 125.41, 124.67, 123.18, $111.63,108.45,107.13,72.06,63.45,56.51,52.47,51.38,44.20,43.80,38.47,35.85,17.96$, 16.84, 14.86. HRMS calculated for $\mathrm{C}_{30} \mathrm{H}_{28} \mathrm{O}_{9}[\mathrm{M}+\mathrm{H}]^{+}: 533.1797$ (found); 533.1806 (calcd). Melting point: $112-115^{\circ} \mathrm{C}$.

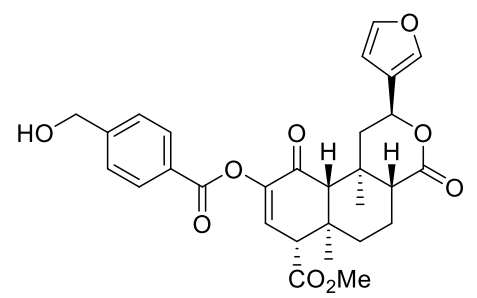

Methyl

(2S,4aR,6aR,7R,10aR,10bR)-2-(furan-3-yl)-9-((4(hydroxymethyl)benzoyl)oxy)-6a,10b-dimethyl-4,10-dioxo-1,4,4a,5,6,6a,7,10,10a,10bdecahydro-2H-benzo[f]isochromene-7-carboxylate (25). ${ }^{1} \mathrm{H}$ NMR $\left(500 \mathrm{MHz}, \mathrm{CDCl}_{3}\right) 8.14$ $8.02(\mathrm{~m}, 2 \mathrm{H}), 7.48(\mathrm{~d}, \mathrm{~J}=8.3 \mathrm{~Hz}, 2 \mathrm{H}), 7.45-7.36(\mathrm{~m}, 2 \mathrm{H}), 6.66(\mathrm{~d}, \mathrm{~J}=2.2 \mathrm{~Hz}, 1 \mathrm{H}), 6.39(\mathrm{dd}, \mathrm{J}=$ 1.9, $0.9 \mathrm{~Hz}, 1 \mathrm{H}), 5.54(\mathrm{dd}, \mathrm{J}=11.5,5.3 \mathrm{~Hz}, 1 \mathrm{H}), 4.80(\mathrm{~d}, \mathrm{~J}=2.6 \mathrm{~Hz}, 2 \mathrm{H}), 3.80(\mathrm{~s}, 3 \mathrm{H}), 3.60(\mathrm{~d}, \mathrm{~J}$ $=2.3 \mathrm{~Hz}, 1 \mathrm{H}), 3.06(\mathrm{dd}, \mathrm{J}=13.7,5.3 \mathrm{~Hz}, 1 \mathrm{H}), 2.47(\mathrm{~s}, 1 \mathrm{H}), 2.24-2.16(\mathrm{~m}, 2 \mathrm{H}), 2.12(\mathrm{dt}, \mathrm{J}=13.4$, $2.7 \mathrm{~Hz}, 1 \mathrm{H}), 1.90(\mathrm{~s}, 1 \mathrm{H}), 1.77-1.64(\mathrm{~m}, 3 \mathrm{H}), 1.37(\mathrm{~s}, 3 \mathrm{H}), 1.24(\mathrm{~s}, 3 \mathrm{H}) .{ }^{13} \mathrm{C}$ NMR $(126 \mathrm{MHz}$, $\left.\mathrm{CDCl}_{3}\right) \delta 190.90,171.40,170.39,164.40,147.14,145.42,143.66,139.37,130.53,129.99$, 127.37, 126.55, 125.40, 108.44, 72.06, 64.57, 63.44, 56.49, 52.48, 51.36, 44.19, 43.79, 38.45, 35.84, 17.94, 16.82, 14.85. HRMS calculated for $\mathrm{C}_{29} \mathrm{H}_{30} \mathrm{O}_{9}[\mathrm{M}+\mathrm{H}]^{+}: 523.1964$ (found); 523.1963 (calcd). Melting point: $115-118^{\circ} \mathrm{C}$.

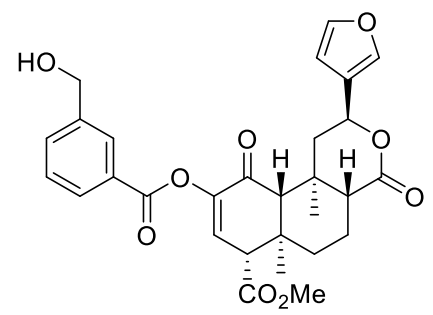

Methyl

(2S,4aR,6aR,7R,10aR,10bR)-2-(furan-3-yl)-9-((3(hydroxymethyl)benzoyl)oxy)-6a,10b-dimethyl-4,10-dioxo-1,4,4a,5,6,6a,7,10,10a,10bdecahydro-2H-benzo[f]isochromene-7-carboxylate (26). ${ }^{1} \mathrm{H}$ NMR (500 MHz, CDCl $) 8.11$ (d, $\mathrm{J}=1.7 \mathrm{~Hz}, 1 \mathrm{H}), 8.03(\mathrm{~d}, \mathrm{~J}=7.8 \mathrm{~Hz}, 1 \mathrm{H}), 7.64(\mathrm{~d}, \mathrm{~J}=7.6 \mathrm{~Hz}, 1 \mathrm{H}), 7.48(\mathrm{t}, \mathrm{J}=7.7 \mathrm{~Hz}, 1 \mathrm{H}), 7.43-$ $7.37(\mathrm{~m}, 2 \mathrm{H}), 6.66(\mathrm{~d}, \mathrm{~J}=2.1 \mathrm{~Hz}, 1 \mathrm{H}), 6.39(\mathrm{~d}, \mathrm{~J}=1.8 \mathrm{~Hz}, 1 \mathrm{H}), 5.54(\mathrm{dd}, \mathrm{J}=11.5,5.3 \mathrm{~Hz}, 1 \mathrm{H})$, $4.77(\mathrm{~s}, 2 \mathrm{H}), 3.80(\mathrm{~s}, 3 \mathrm{H}), 3.60(\mathrm{~d}, \mathrm{~J}=2.1 \mathrm{~Hz}, 1 \mathrm{H}), 3.06(\mathrm{dd}, \mathrm{J}=13.7,5.4 \mathrm{~Hz}, 1 \mathrm{H}), 2.47(\mathrm{~s}, 1 \mathrm{H})$, $2.24-2.07(\mathrm{~m}, 3 \mathrm{H}), 1.85(\mathrm{~s}, 1 \mathrm{H}), 1.77-1.63(\mathrm{~m}, 3 \mathrm{H}), 1.37(\mathrm{~s}, 3 \mathrm{H}), 1.24(\mathrm{~s}, 3 \mathrm{H}) .{ }^{13} \mathrm{C}$ NMR $(126$ 
$\mathrm{MHz}, \mathrm{CDCl} 3) \delta 190.87,171.38,170.37,164.47,145.45,143.67,141.56,139.38,132.31,129.98$, $129.49,128.90,128.56,128.52,125.44,108.45,72.06,64.63,63.49,56.51,52.46,51.38,44.22$, 43.82, 38.48, 35.86, 17.97, 16.82, 14.86. HRMS calculated for $\mathrm{C}_{29} \mathrm{H}_{30} \mathrm{O}_{9}[\mathrm{M}+\mathrm{H}]^{+:}: 523.1959$ (found); 523.1963 (calcd). Melting point: $104-106^{\circ} \mathrm{C}$.

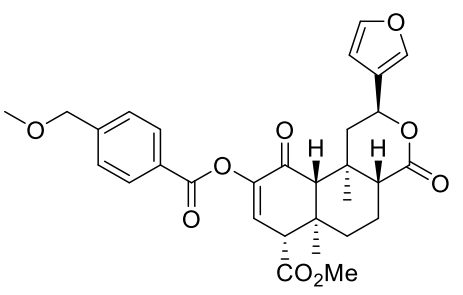

Methyl

(2S,4aR,6aR,7R,10aR,10bR)-2-(furan-3-yl)-9-((4(methoxymethyl)benzoyl)oxy)-6a,10b-dimethyl-4,10-dioxo-1,4,4a,5,6,6a,7,10,10a,10bdecahydro-2H-benzo[f]isochromene-7-carboxylate (27). ${ }^{1} \mathrm{H}$ NMR $\left(500 \mathrm{MHz}, \mathrm{CDCl}_{3}\right) 8.17$ $8.00(\mathrm{~m}, 2 \mathrm{H}), 7.47-7.43(\mathrm{~m}, 2 \mathrm{H}), 7.41(\mathrm{dd}, \mathrm{J}=1.6,0.9 \mathrm{~Hz}, 1 \mathrm{H}), 7.39(\mathrm{t}, \mathrm{J}=1.7 \mathrm{~Hz}, 1 \mathrm{H}), 6.66(\mathrm{~d}$, $\mathrm{J}=2.1 \mathrm{~Hz}, 1 \mathrm{H}), 6.39(\mathrm{dd}, \mathrm{J}=1.9,0.9 \mathrm{~Hz}, 1 \mathrm{H}), 5.60-5.48(\mathrm{~m}, 1 \mathrm{H}), 4.54(\mathrm{~s}, 2 \mathrm{H}), 3.80(\mathrm{~s}, 3 \mathrm{H})$, $3.60(\mathrm{~d}, \mathrm{~J}=2.3 \mathrm{~Hz}, 1 \mathrm{H}), 3.42(\mathrm{~s}, 3 \mathrm{H}), 3.07(\mathrm{dd}, \mathrm{J}=13.7,5.3 \mathrm{~Hz}, 1 \mathrm{H}), 2.47(\mathrm{~s}, 1 \mathrm{H}), 2.20$ (ddd, J = 12.2, 6.4, 3.1 Hz, 2H), 2.12 (dt, J = 13.1, 2.7 Hz, 1H), $1.80-1.62(\mathrm{~m}, 3 \mathrm{H}), 1.38(\mathrm{~s}, 3 \mathrm{H}), 1.24(\mathrm{~s}$, 3H). ${ }^{13} \mathrm{C}$ NMR $\left(126 \mathrm{MHz}, \mathrm{CDCl}_{3}\right) \delta 190.90,171.38,170.38,164.42,145.43,144.74,143.66$, 139.36, 130.42, 129.97, 127.42, 127.28, 125.41, 108.44, 73.90, 72.05, 63.45, 58.45, 56.49, 52.47, 51.37, 44.19, 43.80, 38.45, 35.84, 17.95, 16.82, 14.85. HRMS calculated for $\mathrm{C}_{30} \mathrm{H}_{32} \mathrm{O}_{9}[\mathrm{M}+\mathrm{Na}]^{+}$: 559.1922 (found); 559.1944 (calcd). Melting point: $135-140{ }^{\circ} \mathrm{C}$.

General procedure for MOM-protection of benzoic acids: Protection of the phenolic acids were accomplished following the published protection method. ${ }^{4}$ To a solution of 3 'X4'hydroxybenzoic acid $(1.6 \mathrm{mmol})$ in DCM $(5 \mathrm{~mL})$ stirring under $\mathrm{Ar}$ at $0{ }^{\circ} \mathrm{C}$ was added $\mathrm{N}, \mathrm{N}$ diisopropylethylamine (DIPEA) $(1.5 \mathrm{~mL}, 5.5 \mathrm{eq}$.) followed by the dropwise addition of methoxymethylchloride $(0.7 \mathrm{~mL}, 5.5 \mathrm{eq}$.). This reaction was allowed to stir at r.t. for $48 \mathrm{~h}$ before being quenched with sat. aq. $\mathrm{NH}_{4} \mathrm{Cl}$ solution $(20 \mathrm{~mL})$ and extracted into DCM (3x). The combined organic layers were dried over $\mathrm{Na}_{2} \mathrm{SO}_{4}$ and the solvent was removed under reduced pressure to afford the crude ether ester. To a solution of the crude ester in $\mathrm{MeOH}(20 \mathrm{~mL})$, a $15 \%$ aq. $\mathrm{NaOH}$ solution was added and the mixture was heated for $70^{\circ} \mathrm{C}$ for $3 \mathrm{~h}$. After cooling the reaction to 0 ${ }^{\circ} \mathrm{C}$, the $\mathrm{pH}$ was adjusted to 5 with $6 \mathrm{M} \mathrm{HCl}$ to precipitate a white solid. This solid was filtered to give the desired MOM-protected phenolic acid.<smiles>COc1ccc(C(=O)O)cc1F</smiles>

3-Fluoro-4-(methoxymethoxy)benzoic acid. ${ }^{1} \mathrm{H}$ NMR $\left(400 \mathrm{MHz}, \mathrm{CDCl}_{3}\right)$ $7.90-7.80(\mathrm{~m}, 2 \mathrm{H}), 7.26(\mathrm{~d}, \mathrm{~J}=16.5 \mathrm{~Hz}, 1 \mathrm{H}), 5.31(\mathrm{~s}, 2 \mathrm{H}), 3.53(\mathrm{~s}, 3 \mathrm{H}) .{ }^{13} \mathrm{C} \mathrm{NMR}(101 \mathrm{MHz}$, DMSO) $\delta$ 165.99, 151.48 (d, J = 245.0 Hz), 148.21 (d, J = 10.7 Hz), 126.44 (d, J = 3.1 Hz), 124.69 $(d, J=6.2 \mathrm{~Hz}), 116.85(\mathrm{~d}, \mathrm{~J}=19.6 \mathrm{~Hz}), 116.67,94.67,56.09$. HRMS calculated for $\mathrm{C}_{9} \mathrm{H}_{9} \mathrm{O}_{4} \mathrm{~F}$ [M$\mathrm{H}]^{-}: 199.0457$ (found); 199.0412 (calcd). Melting point: $163-164{ }^{\circ} \mathrm{C}$.<smiles>COc1ccc(C(=O)O)cc1Cl</smiles>

$8.14(\mathrm{~d}, \mathrm{~J}=2.1 \mathrm{~Hz}, 1 \mathrm{H}), 7.97(\mathrm{dd}, \mathrm{J}=8.8,2.1 \mathrm{~Hz}, 1 \mathrm{H}), 7.24(\mathrm{~d}, \mathrm{~J}=8.7 \mathrm{~Hz}, 1 \mathrm{H}), 5.34(\mathrm{~s}, 2 \mathrm{H}), 3.53$ $(\mathrm{s}, 3 \mathrm{H}) .{ }^{13} \mathrm{C} \operatorname{NMR}\left(101 \mathrm{MHz}, \mathrm{CDCl}_{3}\right) \delta 169.80,157.22,132.43,130.36,123.56,123.29,114.98$, 
94.85, 56.63. HRMS calculated for $\mathrm{C}_{9} \mathrm{H}_{9} \mathrm{O}_{4} \mathrm{Cl}[\mathrm{M}-\mathrm{H}]^{-}: 215.0154$ (found); 215.0117 (calcd). Melting point: $169-170{ }^{\circ} \mathrm{C}$.<smiles>COc1ccc(C(=O)O)cc1Br</smiles>

3-Bromo-4-(methoxymethoxy)benzoic acid. ${ }^{1} \mathrm{H} \mathrm{NMR}\left(400 \mathrm{MHz}, \mathrm{CDCl}_{3}\right) 8.30$ $(\mathrm{d}, \mathrm{J}=2.1 \mathrm{~Hz}, 1 \mathrm{H}), 8.00(\mathrm{dd}, \mathrm{J}=8.7,2.1 \mathrm{~Hz}, 1 \mathrm{H}), 7.20(\mathrm{~d}, \mathrm{~J}=8.7 \mathrm{~Hz}, 1 \mathrm{H}), 5.33(\mathrm{~s}, 2 \mathrm{H}), 3.53(\mathrm{~s}$, 3H). ${ }^{13} \mathrm{C}$ NMR (101 MHz, $\left.\mathrm{CDCl}_{3}\right) \delta 169.88,158.11,135.58,131.09,123.70,114.70,112.51$, 94.81, 56.64. HRMS calculated for $\mathrm{C}_{9} \mathrm{H}_{9} \mathrm{O}_{4} \mathrm{Br}$ [M-H] : 258.9652 (found); 258.9611 (calcd). Melting point: $167-168^{\circ} \mathrm{C}$.<smiles>CC(C)(C)OCc1cccc(C(=O)O)c1</smiles>

3-(((Tert-butyldimethylsilyl)oxy)methyl)benzoic acid. To a mixture of 3hydroxymethyl benzoic acid (304 mg, $2.0 \mathrm{mmol})$ in DMF (4mL) was added tert-Butyldimethylsilyl chloride (724 mg, $4.8 \mathrm{mmol})$, and imidazaole (300 mg, $4.4 \mathrm{mmol})$. The reaction mixture was stirred at r.t. overnight before being quenched with acetone $(20 \mathrm{~mL})$. The reaction mixture was chilled in the refrigerator for 20 minutes and then the white precipitate was filtered. The solvent was evaporated, and the crude product was dissolved in THF $(10 \mathrm{~mL})$ and sat. aq. $\mathrm{NaHCO}_{3}$ solution $(10 \mathrm{~mL})$ to hydrolyze the formed silyl ester. The reaction was allowed to stir at r.t. overnight followed by evaporation of the THF. The resulting mixture was acidified with $1 \mathrm{~N} \mathrm{HCl}(30 \mathrm{~mL})$ and extracted with $\mathrm{Et}_{2} \mathrm{O}(3 \times 20 \mathrm{~mL})$. The combined organic extracts were washed with brine $(50 \mathrm{~mL})$, dried $\left(\mathrm{Na}_{2} \mathrm{SO}_{4}\right)$ and concentrated. The resulting clear, pale yellow oil was used without further purification. ${ }^{1} \mathrm{H}$ NMR $\left(400 \mathrm{MHz}, \mathrm{CDCl}_{3}\right) \delta 8.06(\mathrm{dp}, \mathrm{J}=1.5,0.8 \mathrm{~Hz}, 1 \mathrm{H}), 8.00$ (ddt, J = 7.9, 1.9, $1.0 \mathrm{~Hz}, 1 \mathrm{H}$ ), 7.61 (ddq, J = 7.6, 2.0, $0.9 \mathrm{~Hz}, 1 \mathrm{H}), 7.45$ (t, J = 7.7 Hz, 1H), 4.80 (t, J = $0.8 \mathrm{~Hz}, 2 \mathrm{H}$ ), $0.96(\mathrm{~d}, \mathrm{~J}=0.8 \mathrm{~Hz}, 9 \mathrm{H}), 0.12(\mathrm{~d}, \mathrm{~J}=0.6 \mathrm{~Hz}, 6 \mathrm{H}) .{ }^{13} \mathrm{C}$ NMR $\left(101 \mathrm{MHz}, \mathrm{CDCl}_{3}\right) \delta 171.94,142.04$, 131.46, 129.22, 128.80, 128.46, 127.74, 64.46, 25.94, 18.47, -5.25. HRMS calculated for $\mathrm{C}_{14} \mathrm{H}_{22} \mathrm{O}_{3} \mathrm{Si}[\mathrm{M}-\mathrm{H}]^{-}: 265.1302$ (found); 265.1265 (calcd). Melting point: $40-42{ }^{\circ} \mathrm{C}$. 


\section{NMR Spectra}


:

高 高

高

ะ⿳亠口冋้

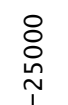

ริำ

章

รั)

高
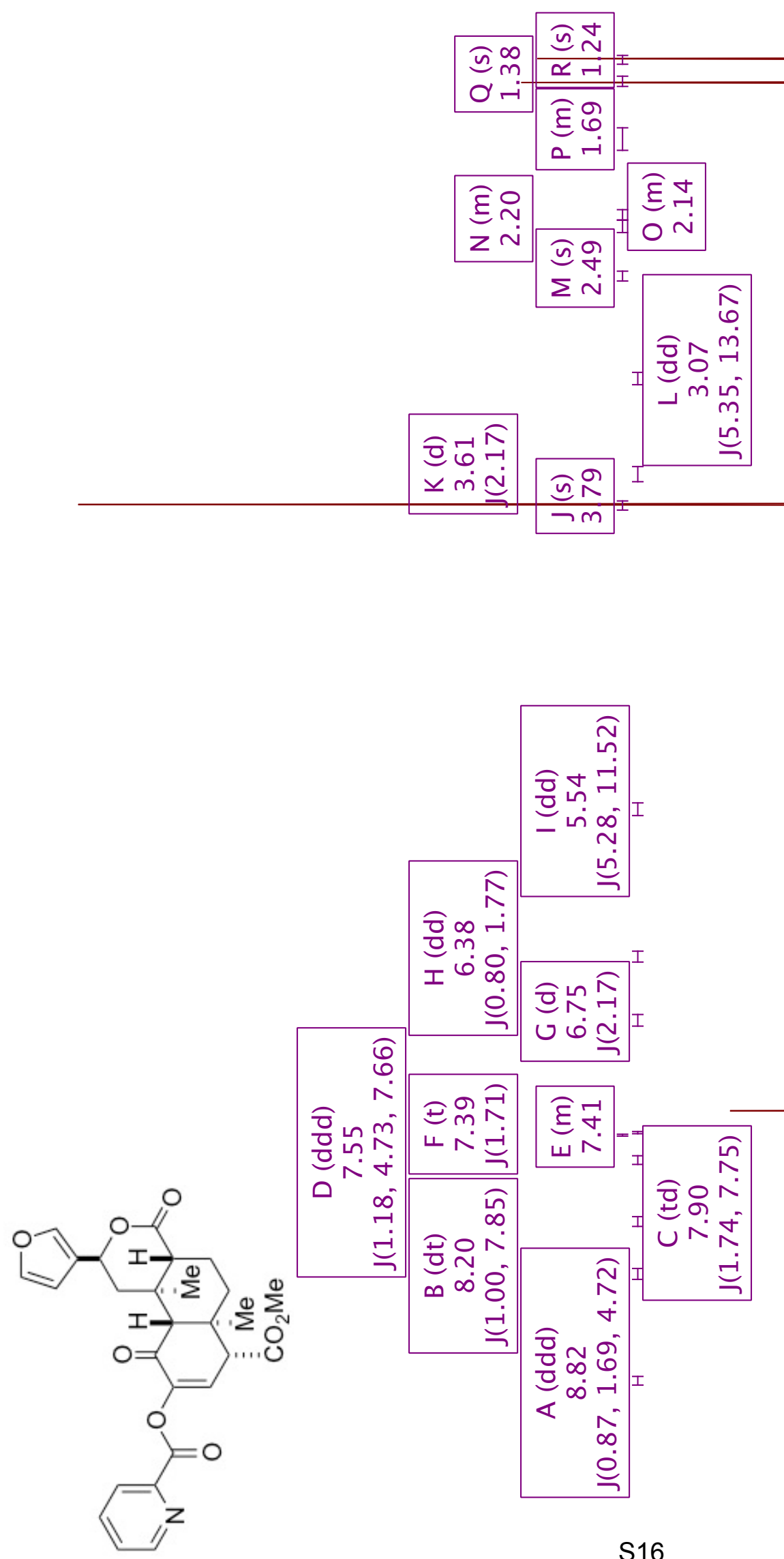


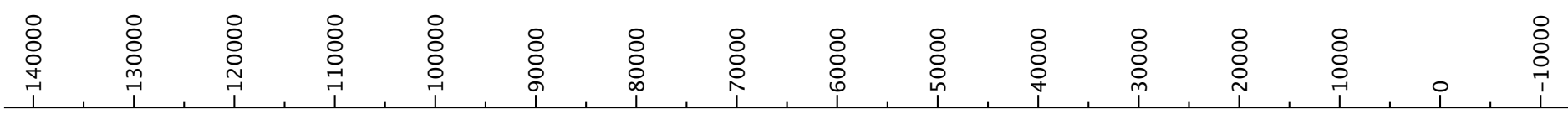

$\varsigma 8^{\circ} \mathrm{L} \mathrm{I}$

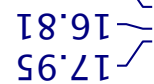

ऽ8. ऽะ-

st.8⿷匚

乙L८ち厂

乙乙一॰

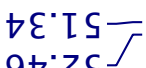

9t.2s

[S.9S

0t'๕9

$\angle 0^{\circ} 2 \angle-$

عt・80I-

st. SZI]

$0 \angle \angle Z I=$

$\varepsilon \varepsilon^{\circ} 0 \varepsilon \tau^{-}$

$\downarrow \tau^{\circ} \angle \varepsilon[ح$

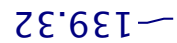

$69^{\circ} \varepsilon \succ \mathrm{I}-$

I $\nabla^{\circ} S t \mathrm{~L}-\tilde{T}$

$0 t^{\circ} 9 t[$

$6 I^{\circ} 0 \mathrm{SI}$

$\downarrow I \cdot \varepsilon 9[-$

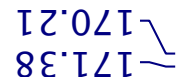

$-$
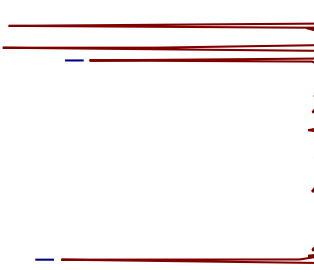

우

in

- 8

$-\circ$

$\infty$

ㅇ

호을

$=$

옥

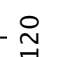

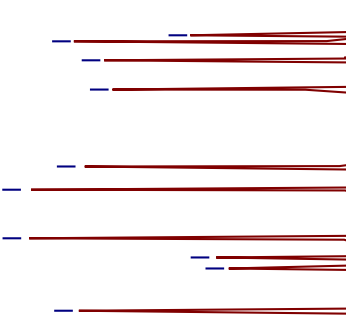

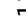

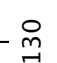

옥

옥

$-8$

옥

옥

$\stackrel{\infty}{-\infty}$

0t.06I- 


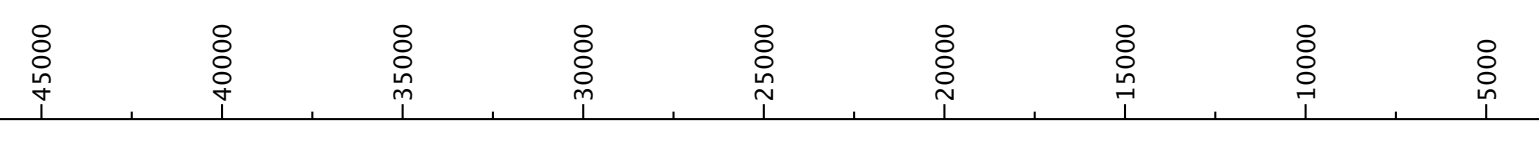
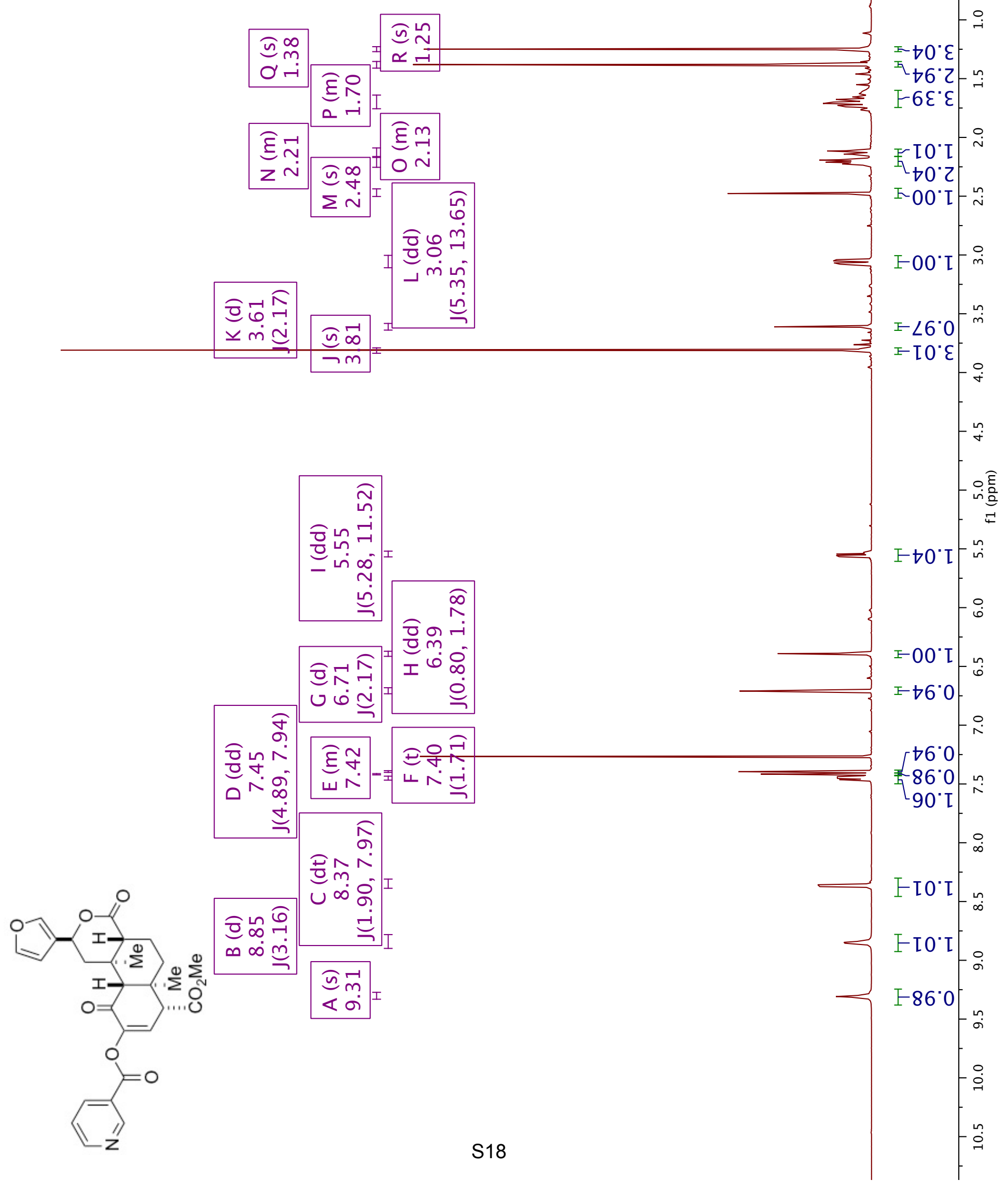


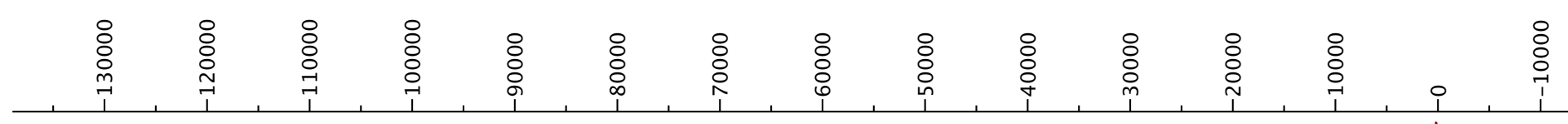

$98^{\circ} \circ \mathrm{L}$

$\varepsilon 8^{\circ} 9 \mathrm{I}-\widetilde{\tau}$
$\succ 6^{\circ} \angle \mathrm{I} J$

88. $\mathrm{s}-$

$7 \nabla^{\circ} 8 \varepsilon^{-}$

$\varepsilon 8^{\circ} \varepsilon \nabla T$

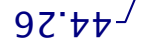

SE'IST

$\rightarrow \mathrm{s} \cdot \mathrm{Ts}$

$97^{\circ} 9 \mathrm{~s}$

0S' $\varepsilon 9-$

$20^{\circ} 2 L-$

$\varepsilon t^{*} 80 \mathrm{I}-$

$0 S^{\circ} \varepsilon Z I_{2}$

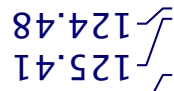

St.0E[ I

$\angle 9^{\circ} \angle \varepsilon I-$

$8 \varepsilon^{\circ} 6 \varepsilon \mathrm{I}^{-}$

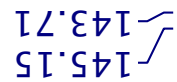

St. ISI-

$6 Z^{\circ}+5 I^{-}$

$8 z^{\circ} \varepsilon 9 \mathrm{I}-$

$92^{\circ} 0<\mathrm{L}$ \

$6 Z^{\circ} \cdot \mathrm{L} \angle \mathrm{I}-$

S9.06I-
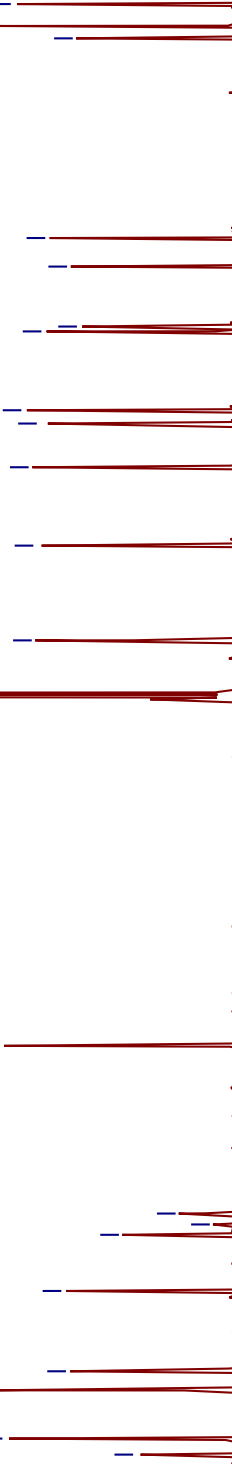


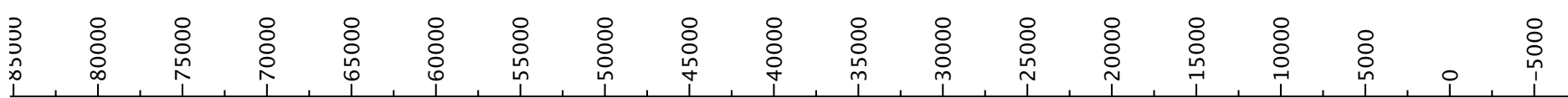

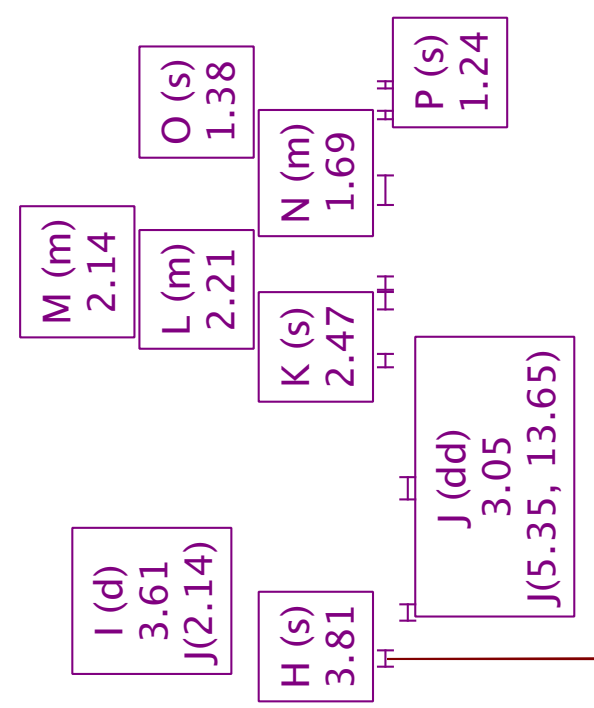

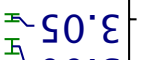

- $00 \cdot \varepsilon-\stackrel{n}{-}$ - $66^{\circ} \mathrm{r}$

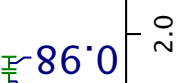

$766^{\circ} \mathrm{L}$

ᄃ $86^{\circ} 0-\stackrel{\sim}{\sim}$

I $56.0-\stackrel{\circ}{m}$
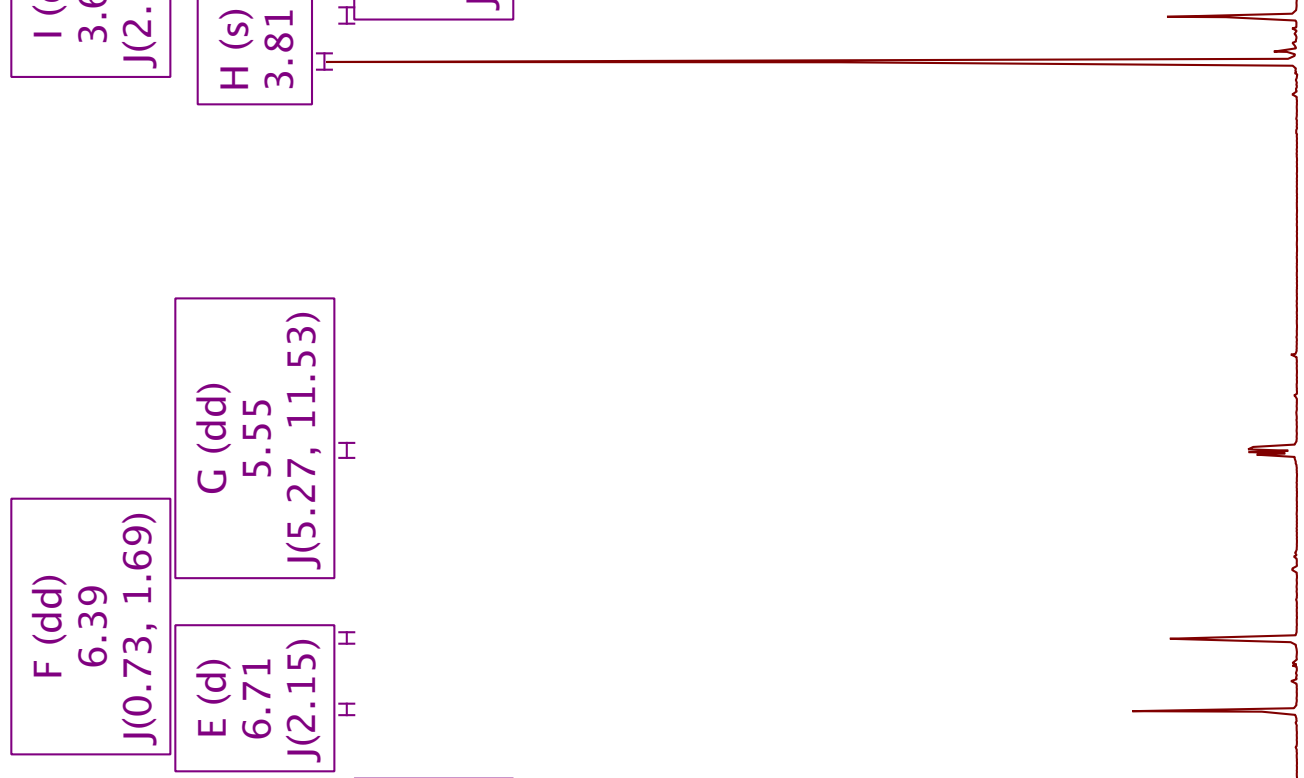

I 76.0

工 $96^{\circ} \mathrm{Z}$
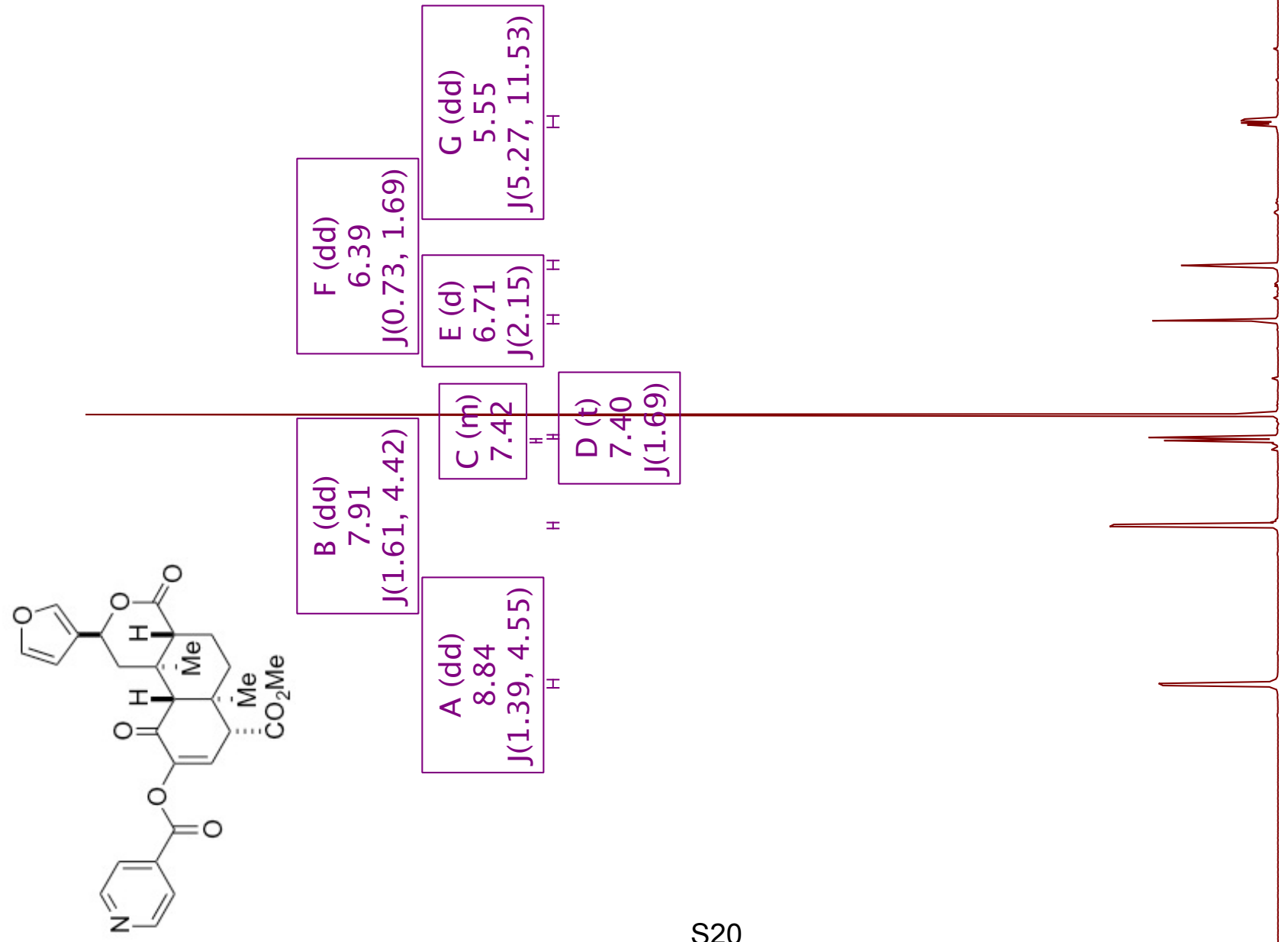

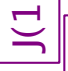

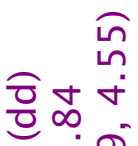

$\varangle \overbrace{}^{\infty} \tilde{m}^{-1}$

닫 


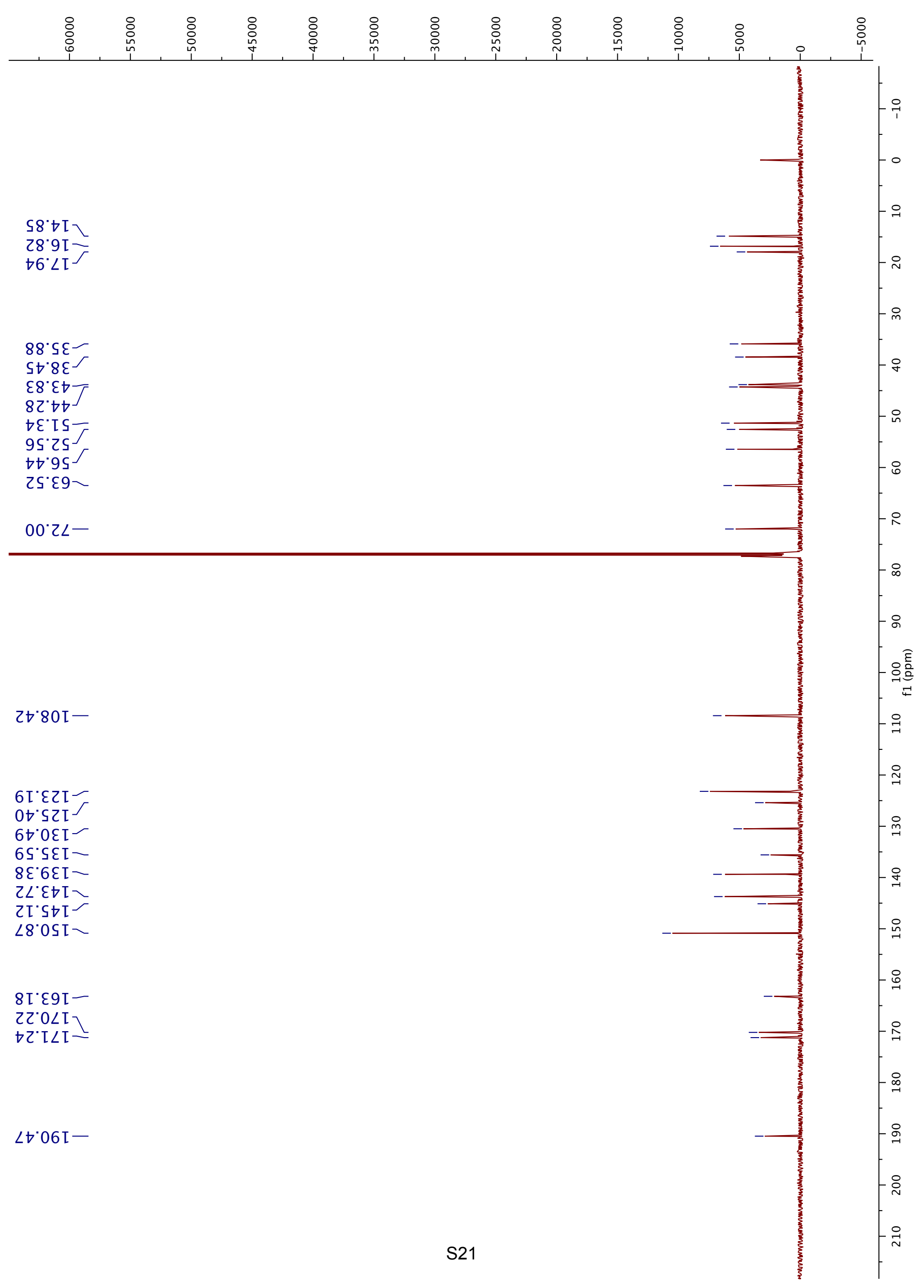



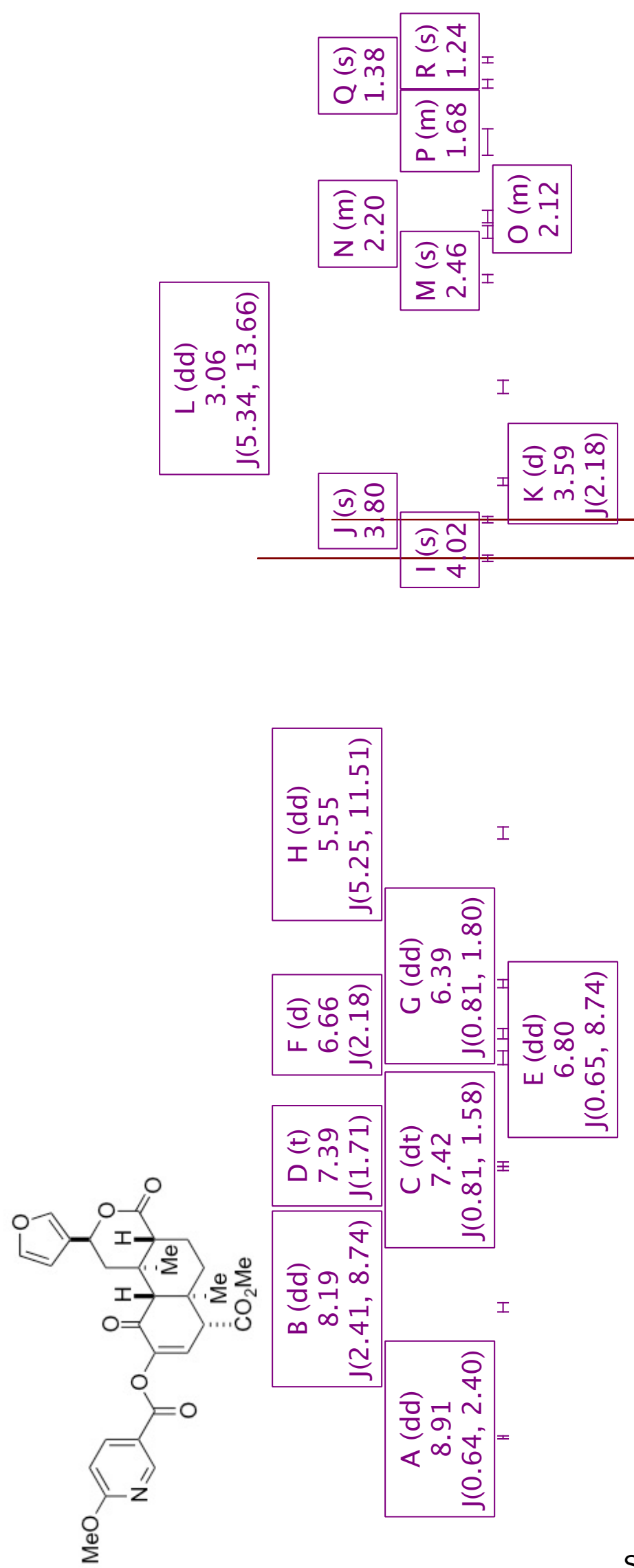
$98^{\circ} \circ \mathrm{I}$

$88^{\circ} 9 \mathrm{I}-$
S6. $\angle \mathrm{I}$

$98^{\circ} \varsigma \varepsilon 乙$

tฤ. $8 \varepsilon-$

$28^{\circ} \varepsilon \downarrow>$

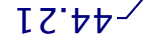

$9 \varepsilon$ 'IS

6t'2s

$6 t^{\circ} 9 \mathrm{~s}$

$8 t^{\circ} \varepsilon 9^{-}$

$70 \cdot 2 L-$
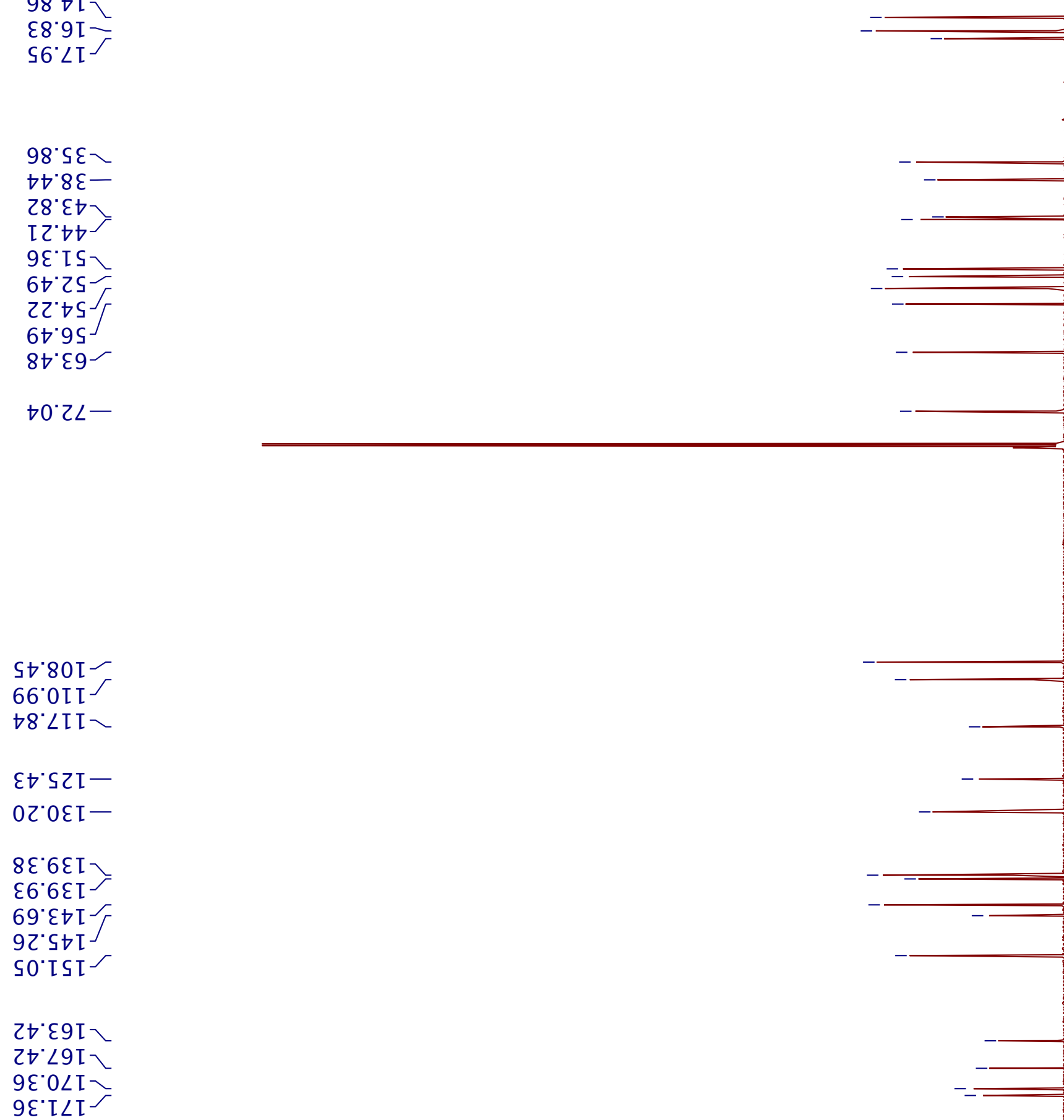


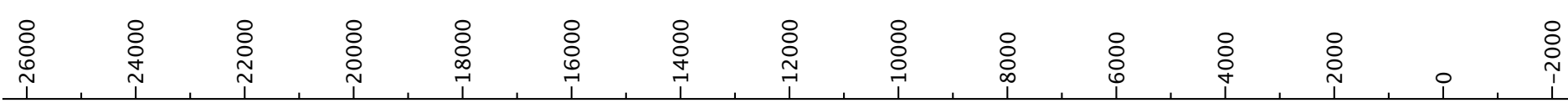
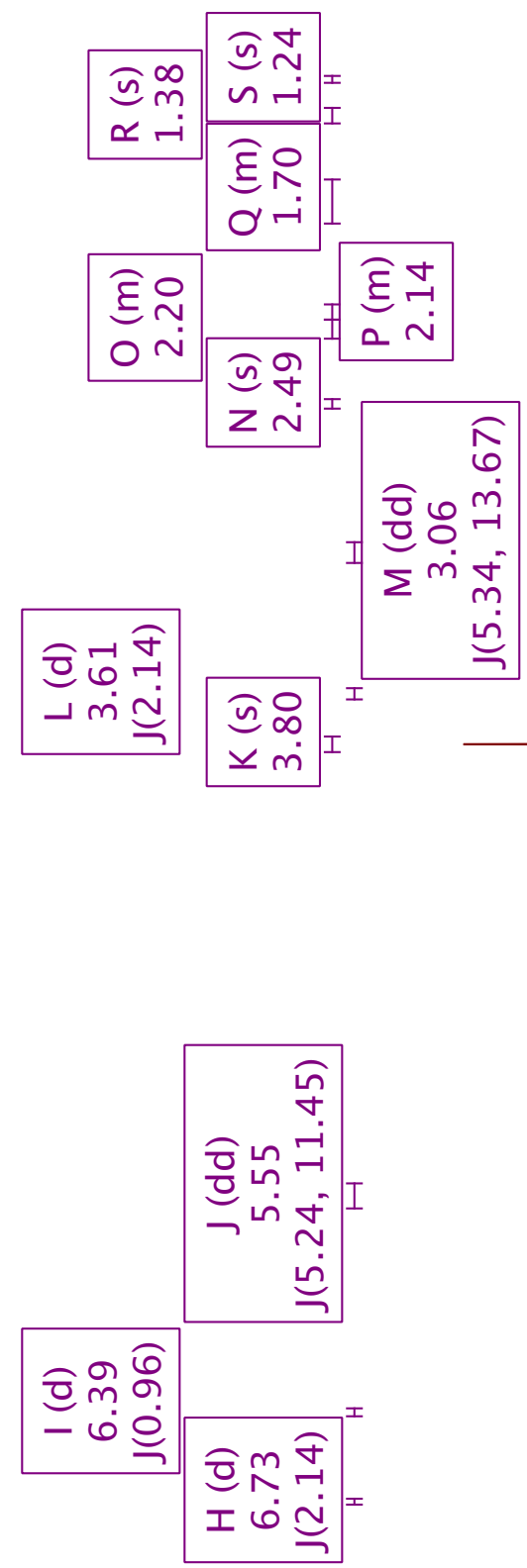

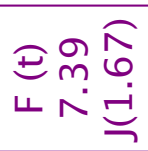

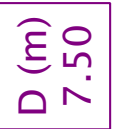

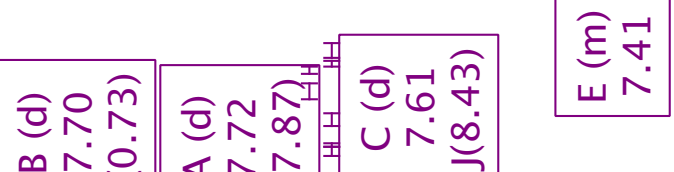

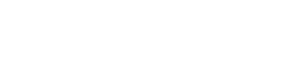


$58^{\circ} \downarrow \mathrm{I} \backslash$

$28^{\circ} 9 \mathrm{I}$
$56^{\circ} \angle \mathrm{I}$

$\downarrow 8^{\circ} \varsigma \varepsilon-$

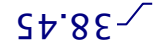

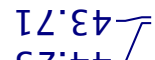

$\varepsilon \iota^{*} \triangleright \nabla^{\top}$

I $\varepsilon^{\circ}$ IS

27.925

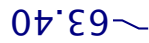

$90^{\circ} 2 \angle-$

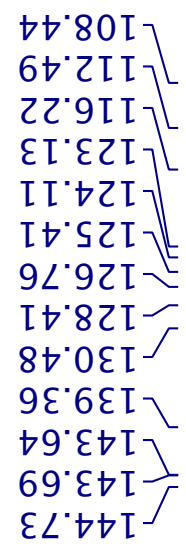

$\angle I^{\circ} 9 S^{\circ}=$
S0 $\angle S I=$

$\nabla 2^{\circ} 0 \angle \mathrm{I}$

$S \varepsilon^{\circ}\left[\angle L^{-}\right.$

$\forall S^{\circ} 06 \mathrm{I}-$

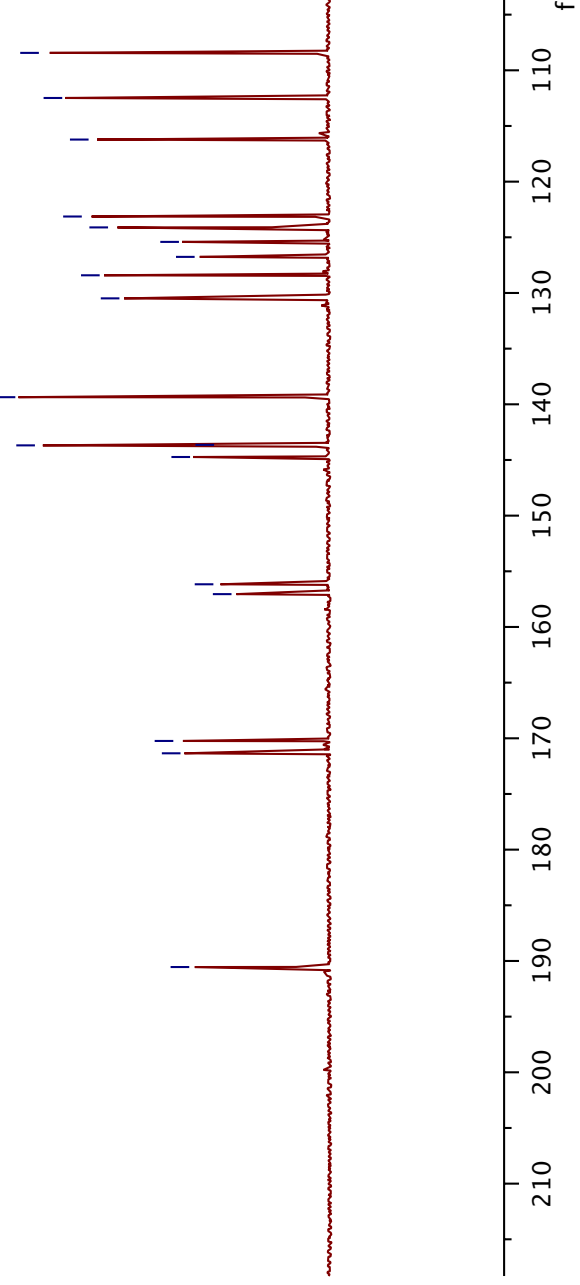




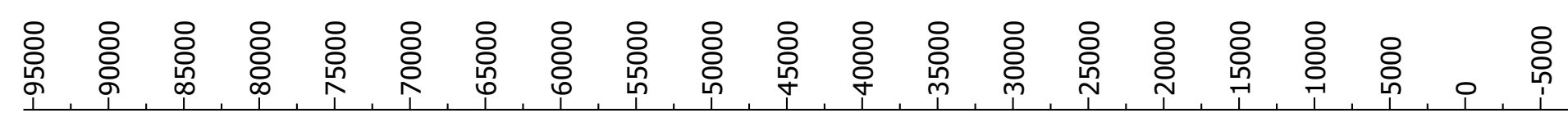
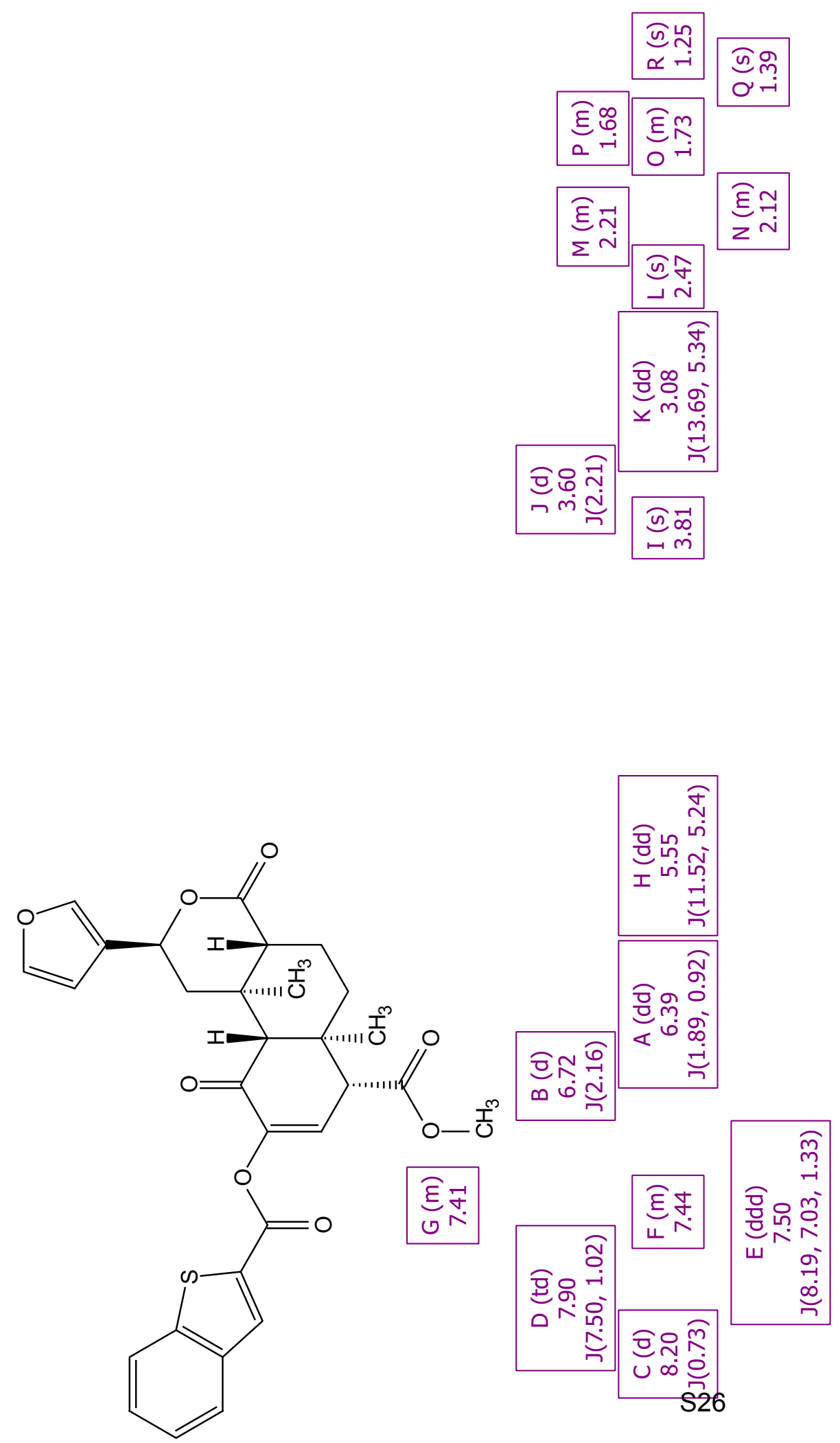


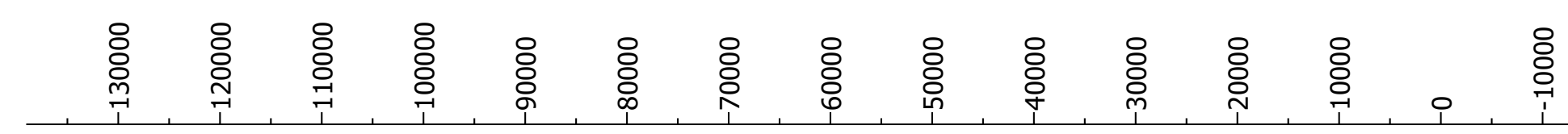

S8'十丁 乙

ع8.9 -

$28.08-$

08' $\mathrm{E} \downarrow$

$2 C^{\circ} t \circ$

SE'IS-

IS'ZS

9t' 95 -

८৮'ย9-

$90^{\circ} Z L-$

tเ'80I -

โ8'22L

9I'SZI ]

Ot.SZI]

${ } S^{\circ} \angle Z I-$

LE'0हI $\bar{\Gamma}$

IS'ZEI

દ૬'8દโ -

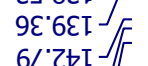

6L'Z†I-

$\angle 9^{\circ} \varepsilon \sqcup[-$

90'StI

tS.09I -

$8 Z^{\circ} 0 \angle \mathrm{I}-$

$9 \varepsilon^{\circ} \mathrm{TLT} \nearrow$

५9.06I -
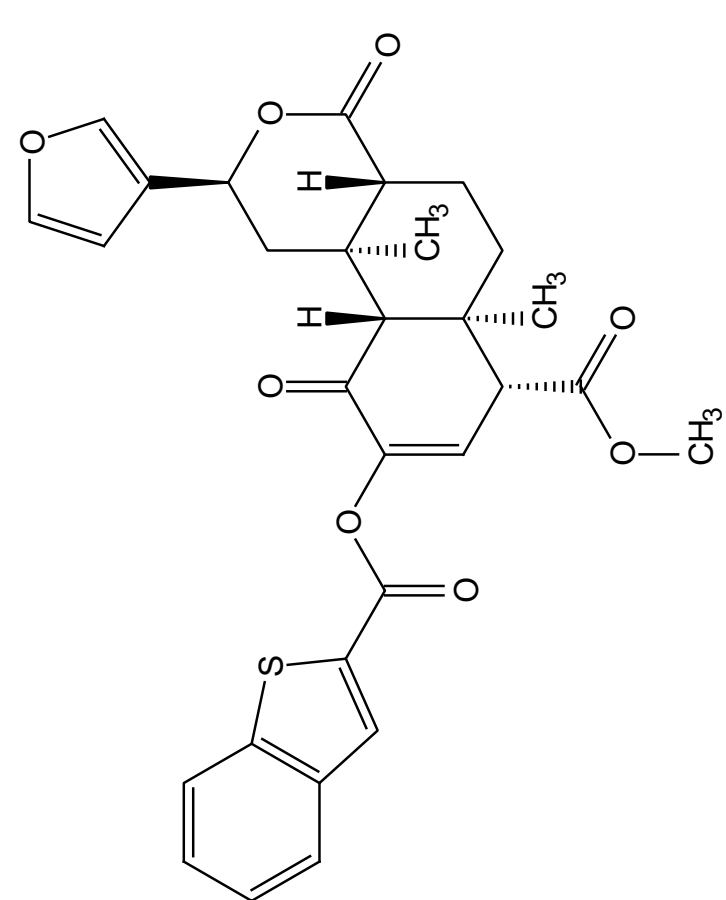

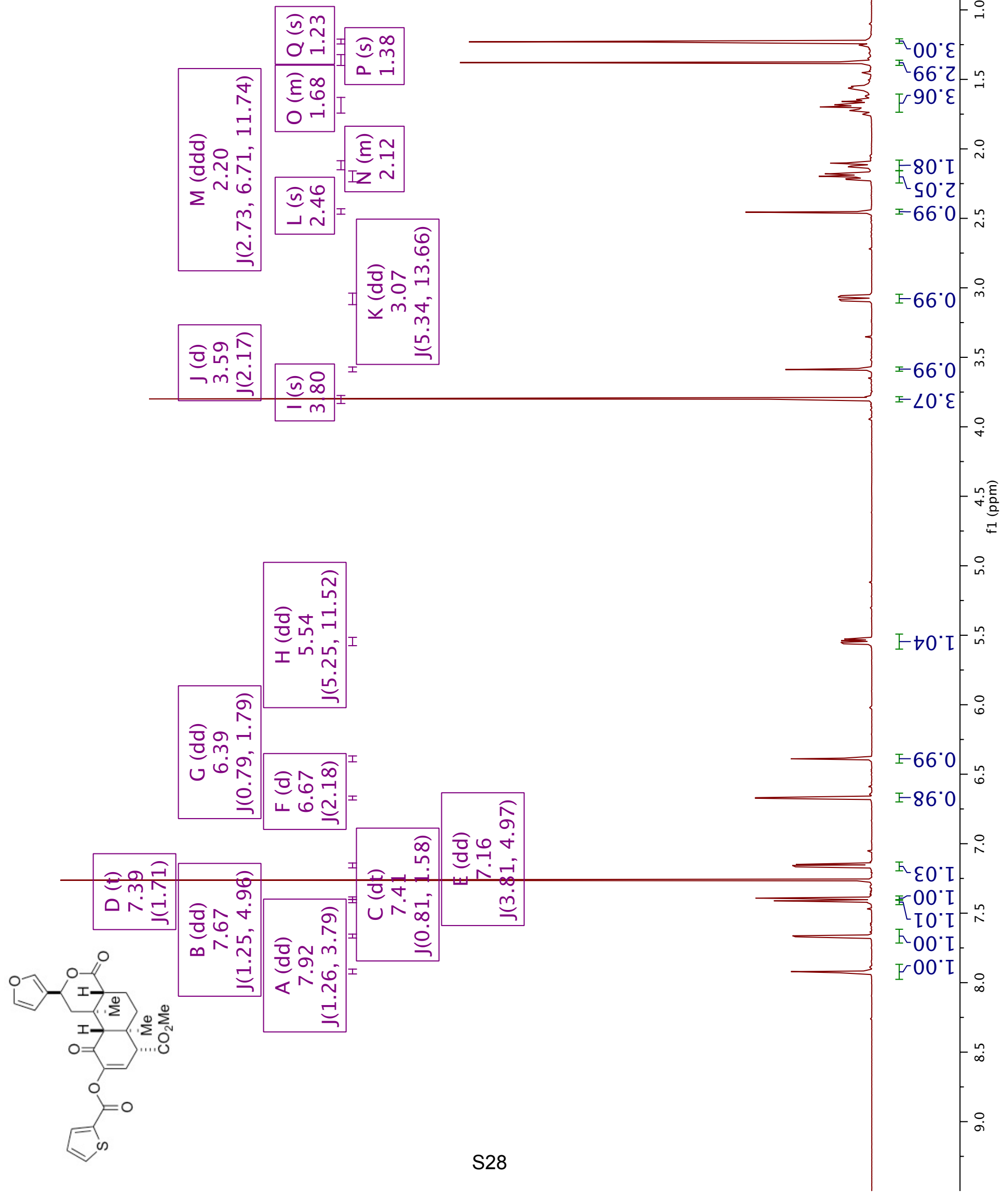
$\left.98^{\circ} \sqcup \mathrm{I}\right\urcorner$

$28^{\circ} 9 \mathrm{I}-$
$56^{\circ} \angle \mathrm{I}$

ऽ $8^{\circ} \varsigma \varepsilon^{-}$

St.8E $8 \varepsilon^{-}$

28. $ॄ \nabla T$

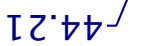

$\angle \varepsilon^{\circ}$ IS

$8 t^{\circ} 25$

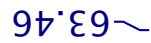

$\angle 0^{\circ} 2 \angle-$

†๑ $80 \mathrm{I}-$

$\varepsilon t^{\circ} \varsigma$ ZI
$\forall[.8 Z I]$

ऽટ'0દL ᄂ

$\neg \varepsilon$ IEI-

80" $\downarrow\llcorner$ โ

$\downarrow Z^{\circ} \varsigma \varepsilon \mathrm{J}$

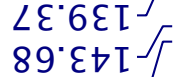

90.งt [

$68^{\circ} 6 \mathrm{SI}-$

$\varepsilon \varepsilon^{\circ} 0 \angle I=$
$\angle \mathcal{E}^{\circ}[\angle T-$

$\varepsilon 8^{\circ} 06 \mathrm{I}-$ 


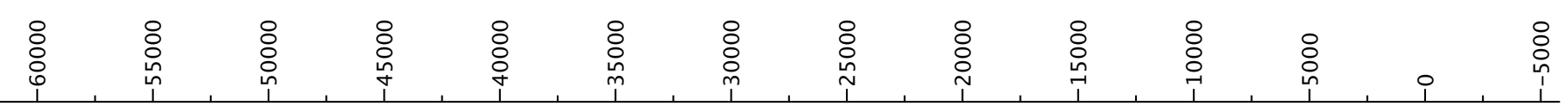
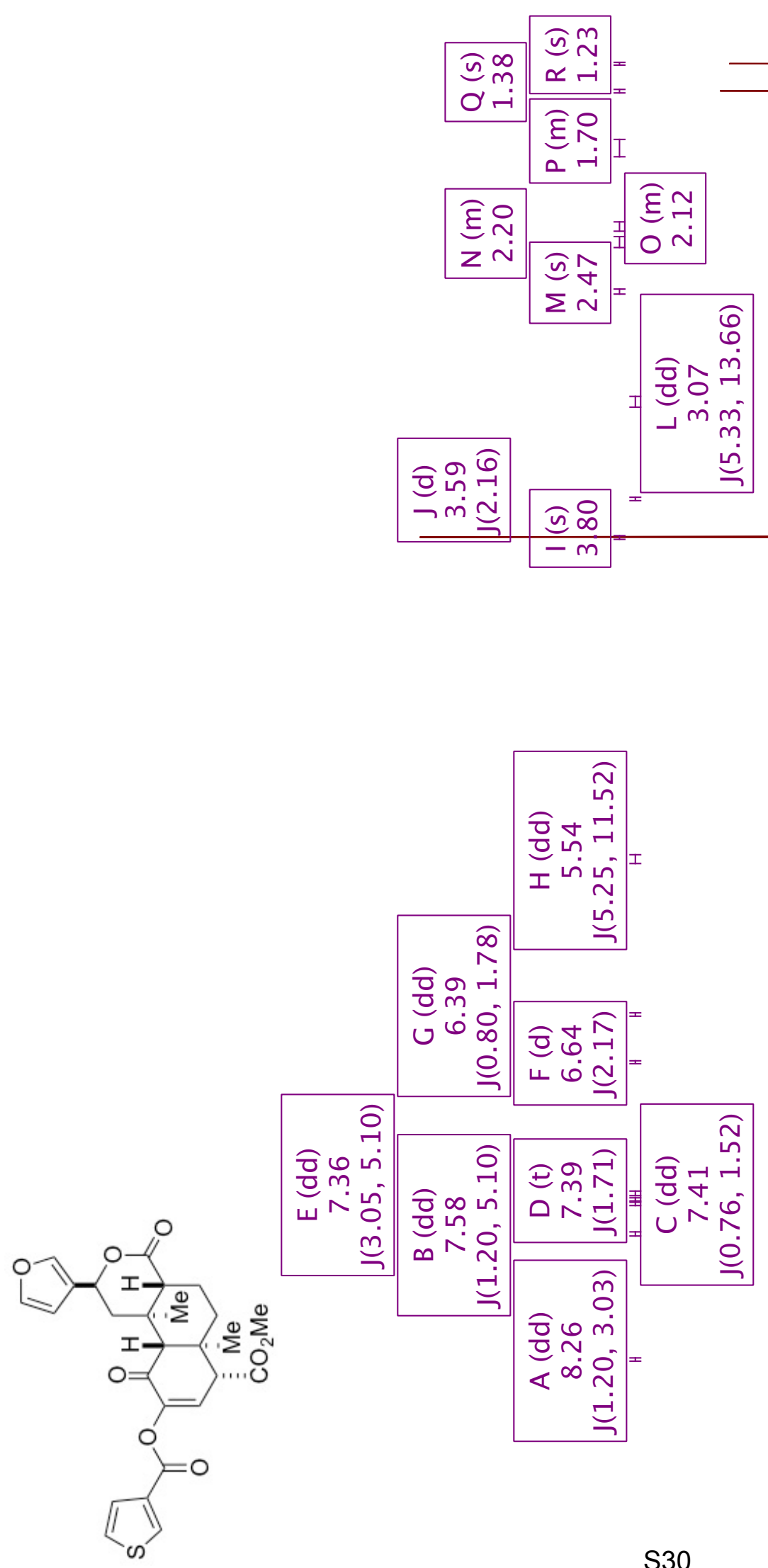


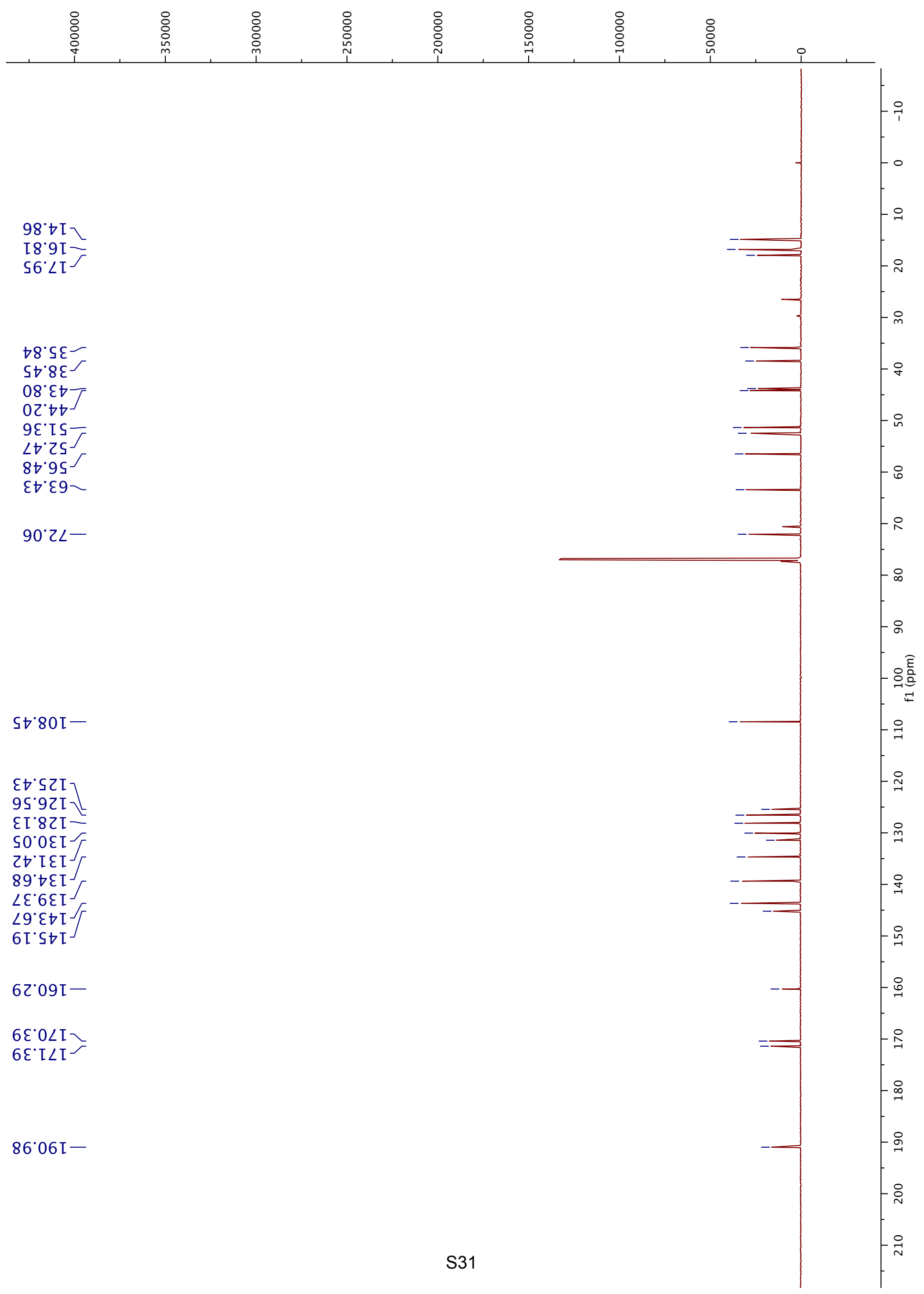



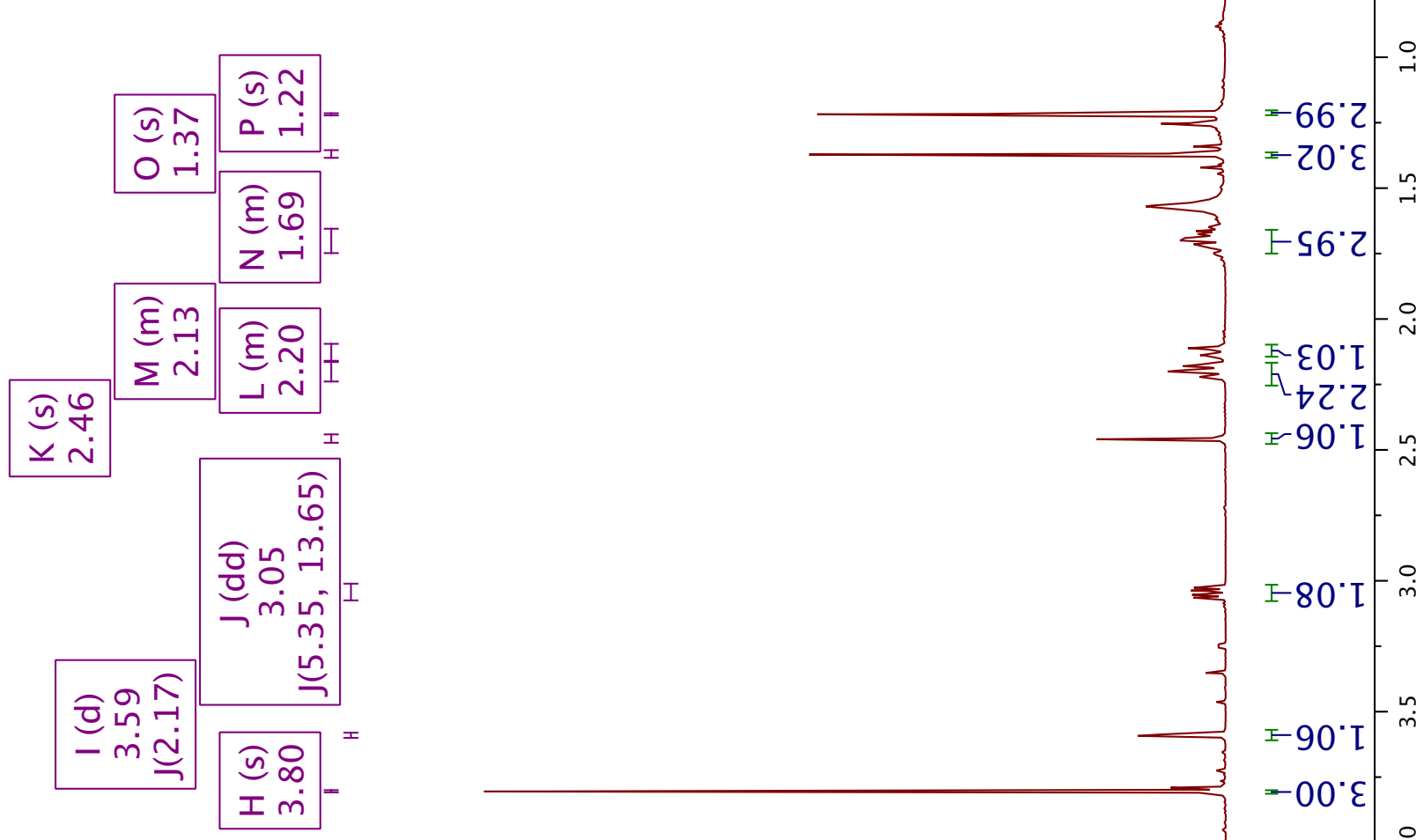

그
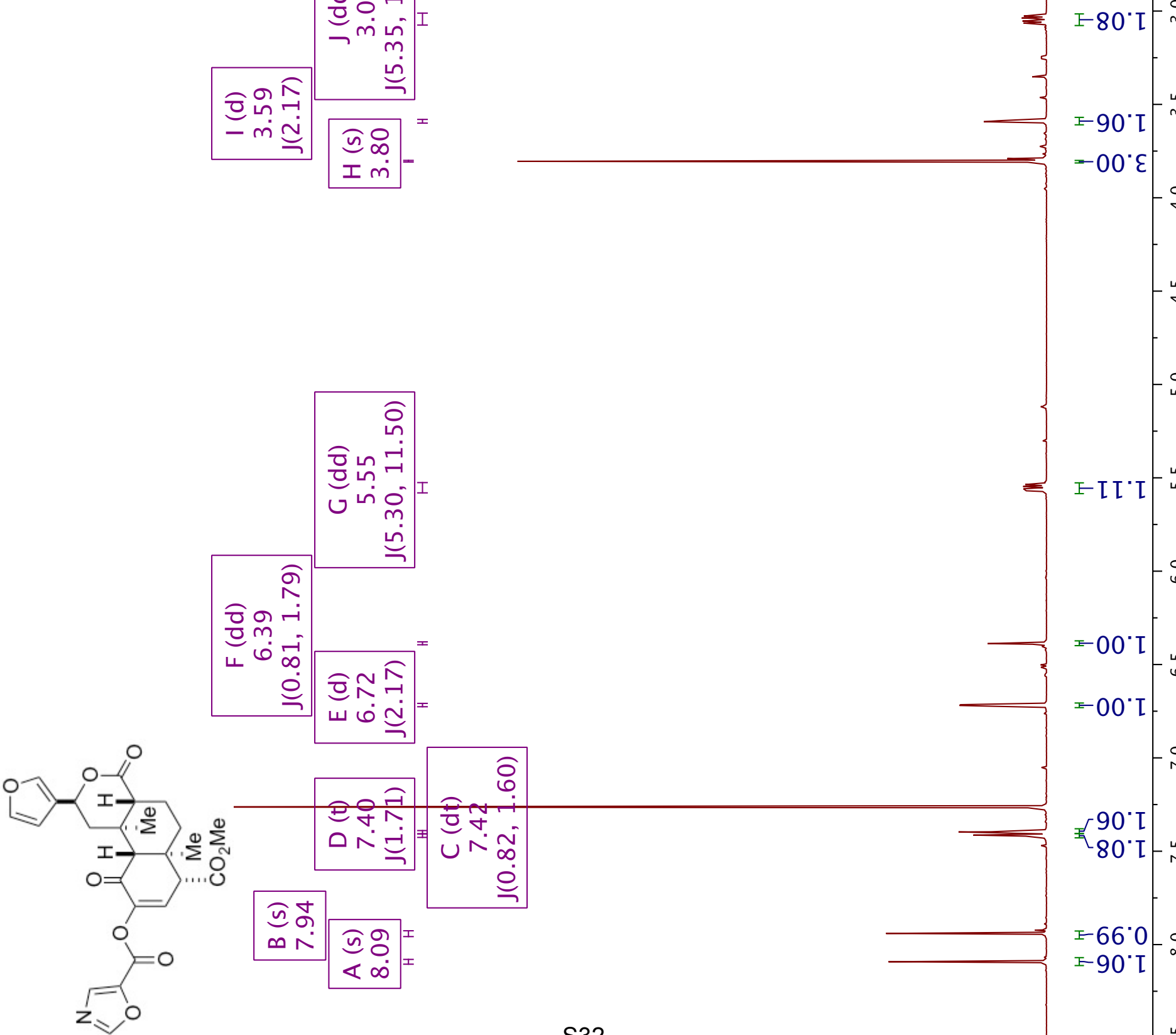


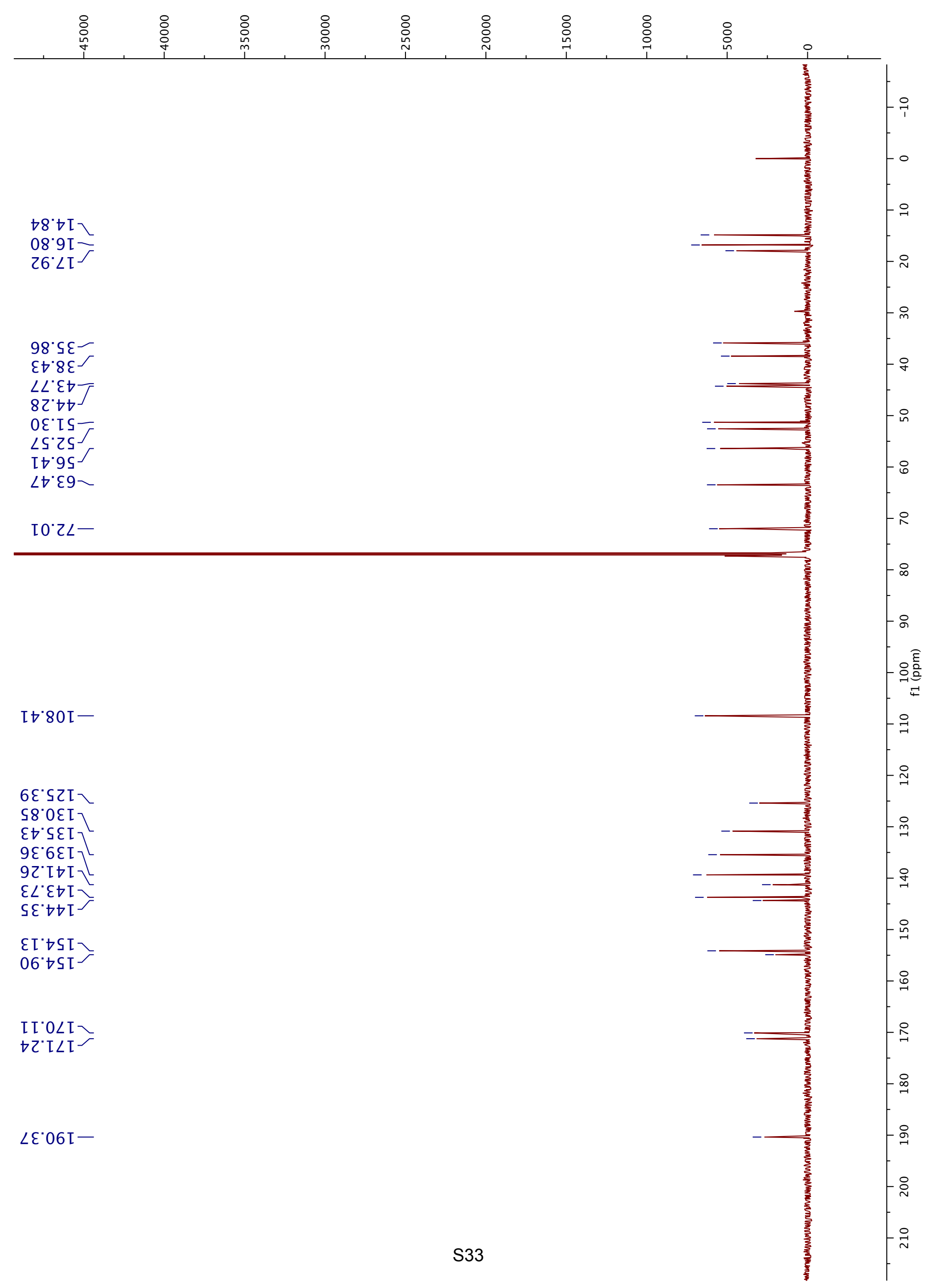




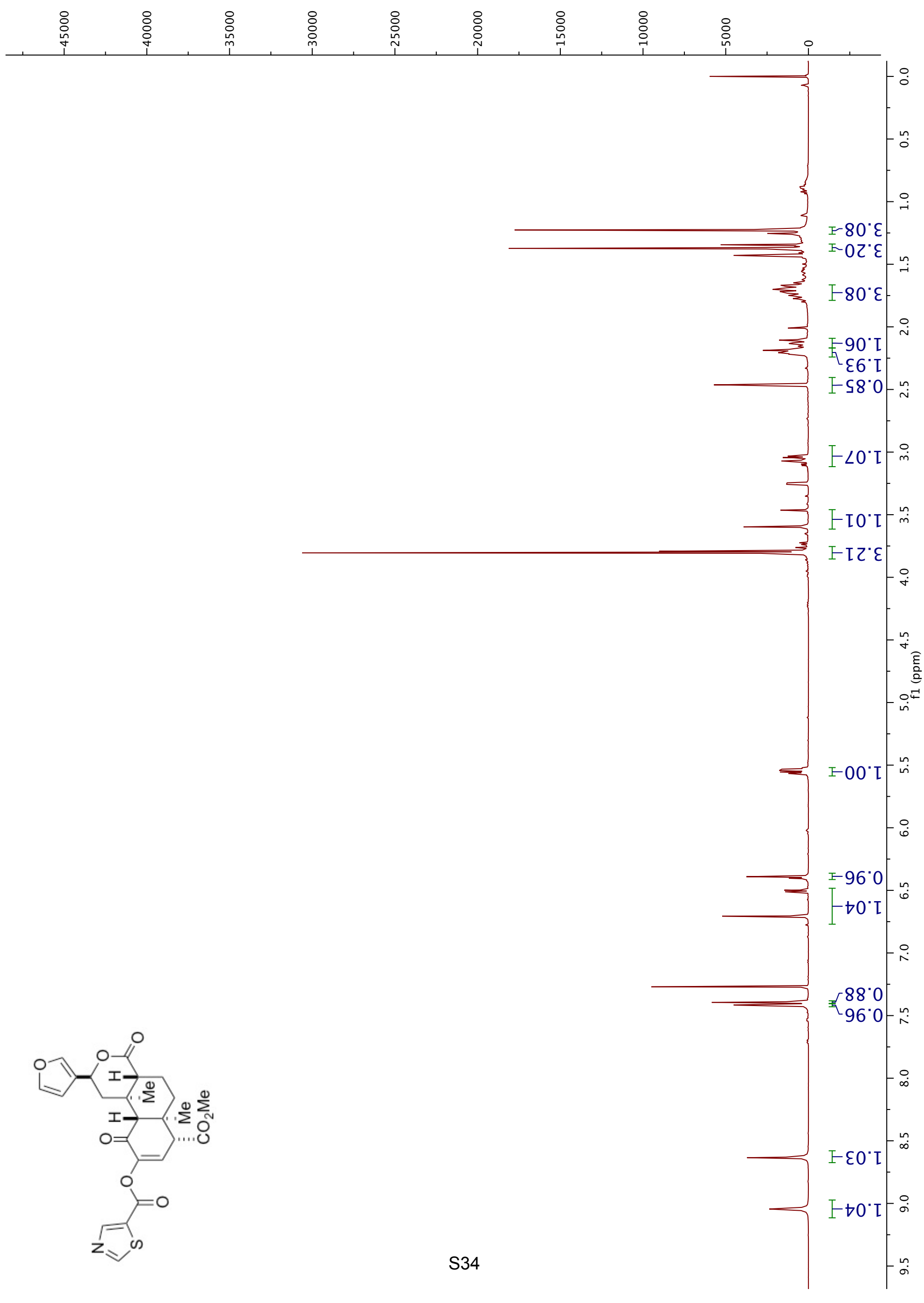


$\forall 8^{\circ} \circ \mathrm{I}$

$28^{\circ} 9 \mathrm{I}-\bar{J}$
$26^{\circ} \angle \mathrm{I}$

98. ऽะ-

$\mathrm{L} \nabla^{\circ} 8 \varepsilon^{-}$

6L ह†T

$9 \tau^{\prime} \bullet t$

0E'IS-

SS'ZS

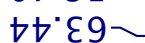

I0.2L-

$\varepsilon t^{\circ} 80 \mathrm{I}-$

$6 \varepsilon^{\circ} \mathrm{SZI}-$

$89^{\circ} 0 \varepsilon \mathrm{I}-$

$8 \varepsilon^{\circ} 6 \varepsilon[-$

L $\angle$ ' $\varepsilon \sqcup \mathrm{I}$

$9 \angle \cdot \nabla \nabla[J$

ES.OSI-

$98^{\circ} 8 \mathrm{SI}$

ع $\varepsilon^{\circ} 6 \mathrm{SI}^{-}$

$6 \mathrm{I}^{\circ} 0<\mathrm{L}$

$95^{\circ} 0 \angle \mathrm{I} \frac{\mathrm{I}}{\mathrm{J}}$

$6 Z^{\circ}[\angle \mathrm{I}$

SS.06I- 
高高

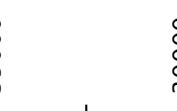

I

sั

高

ํํㄹำ
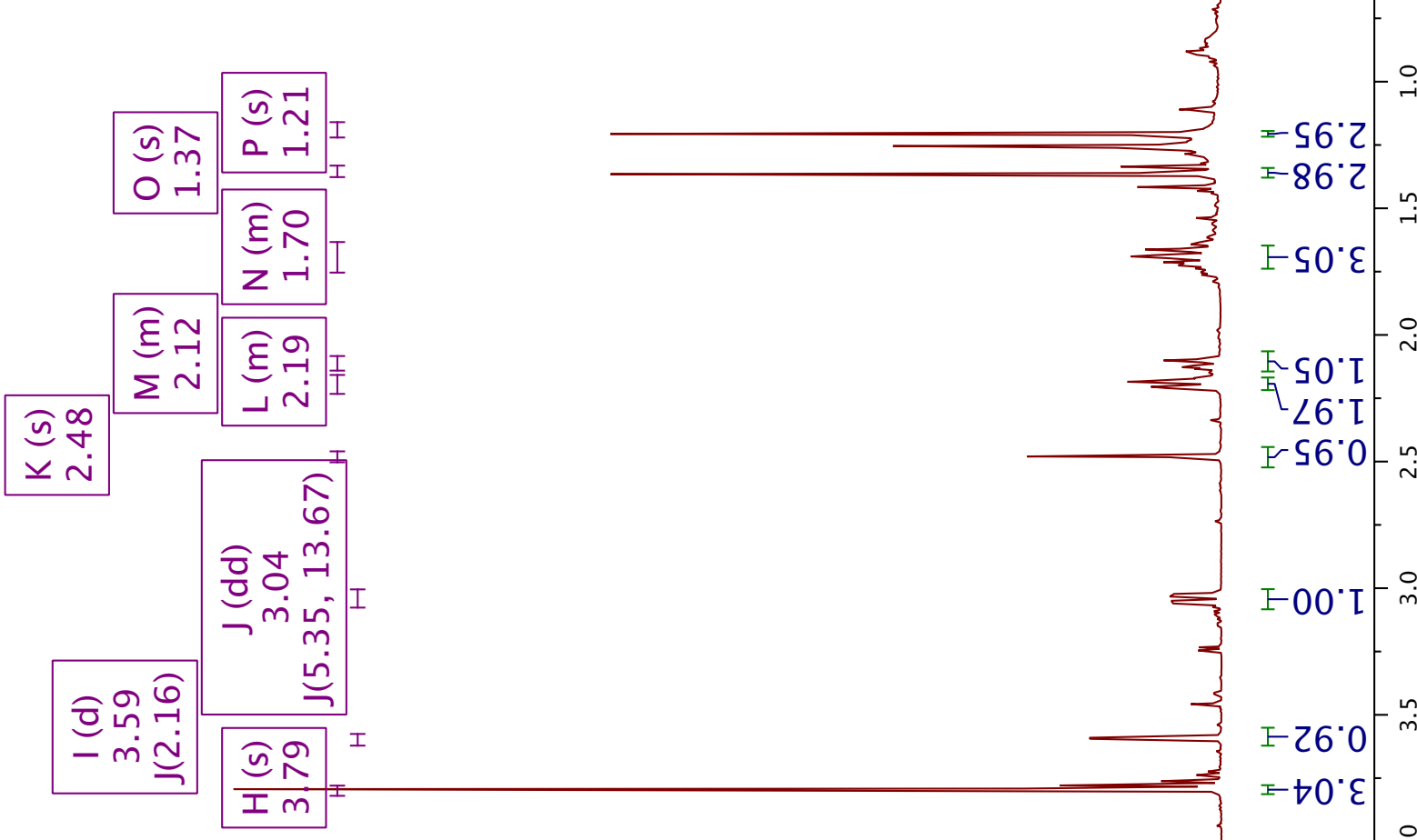

$-86^{\circ} \mathrm{Z}$

$\mp \subseteq 0^{\circ} \varepsilon$

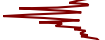

0

ऍ $50^{\circ} \mathrm{L}$

$\angle 6^{\circ} \mathrm{I}$

$-56^{\circ} 0$ in

F $00^{\circ} \mathrm{L}-\stackrel{\circ}{m}$
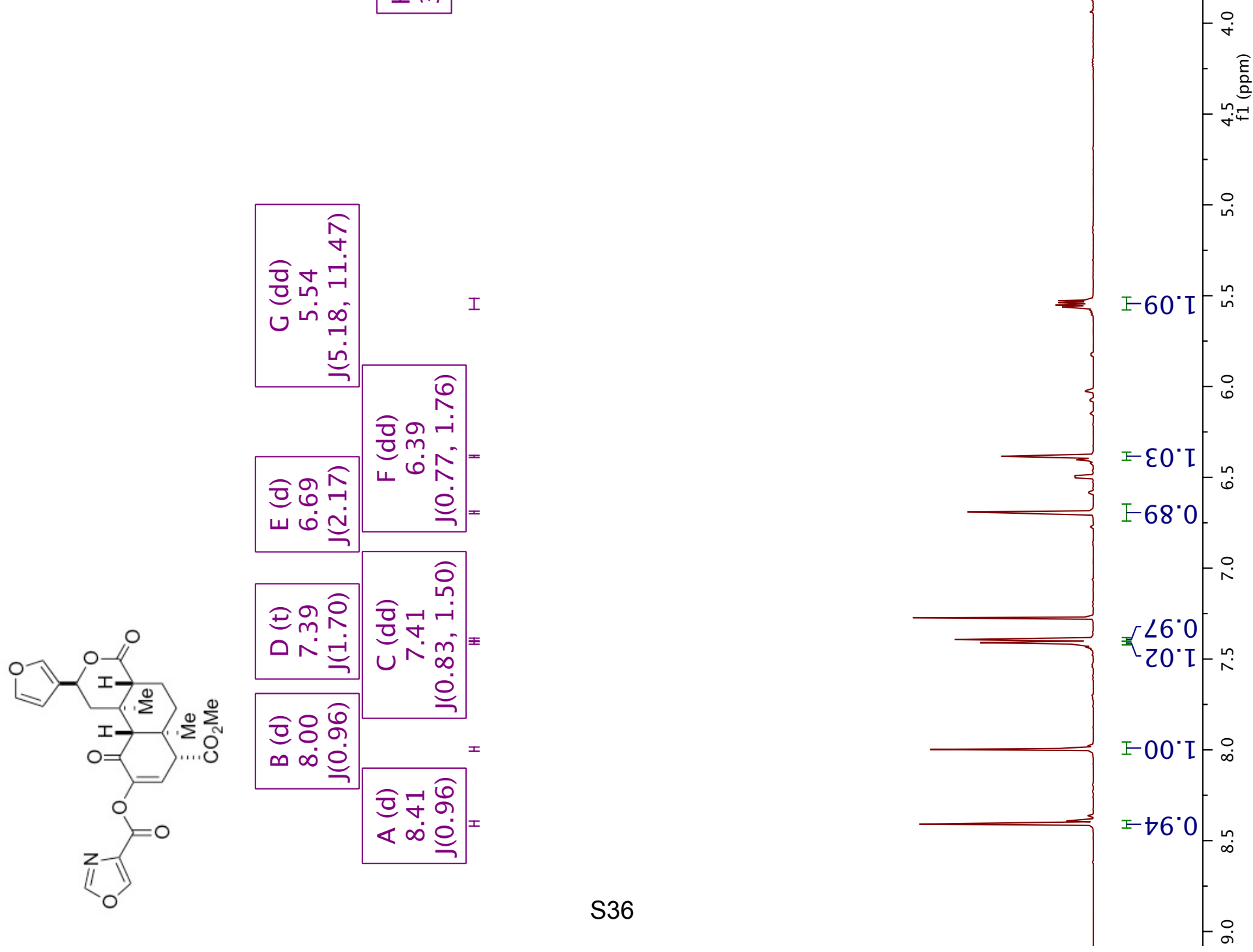


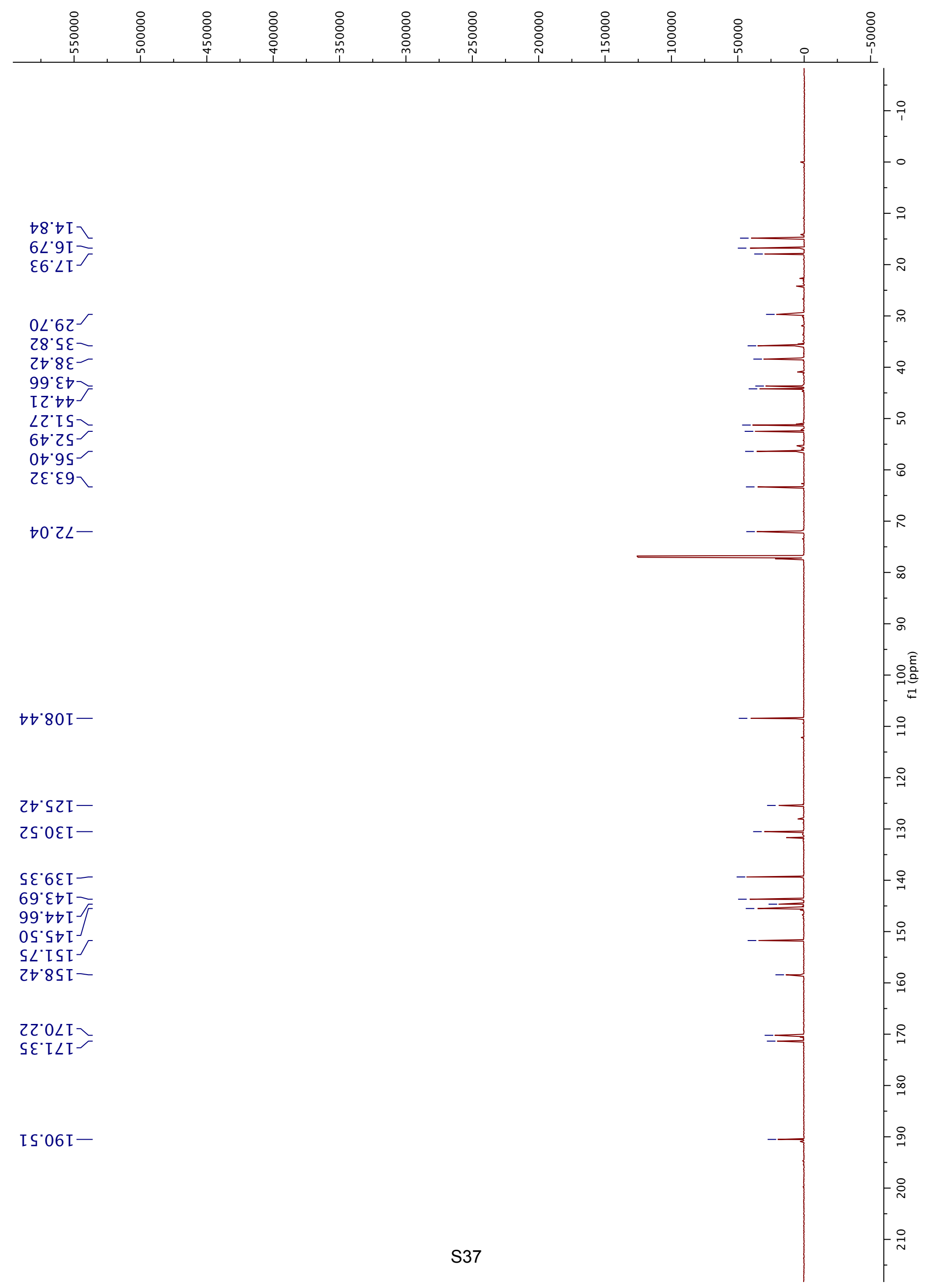


旁高旁,

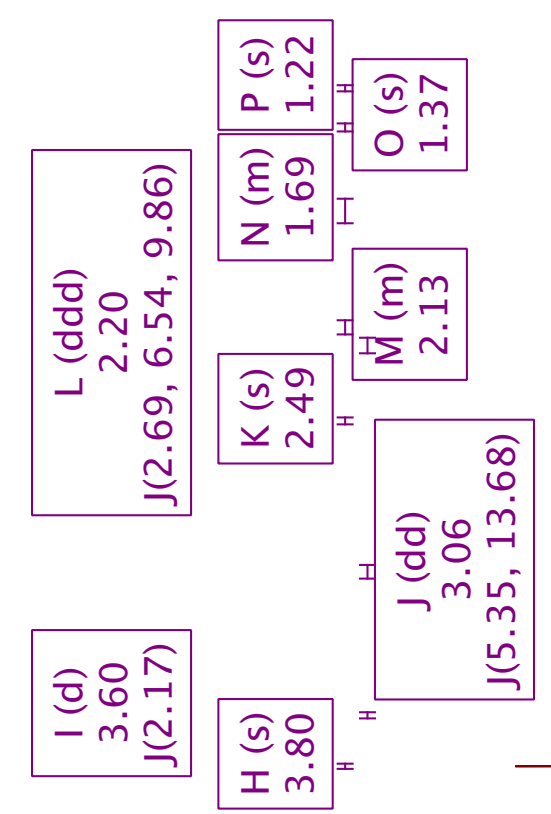

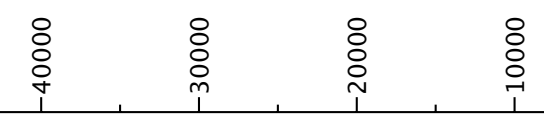

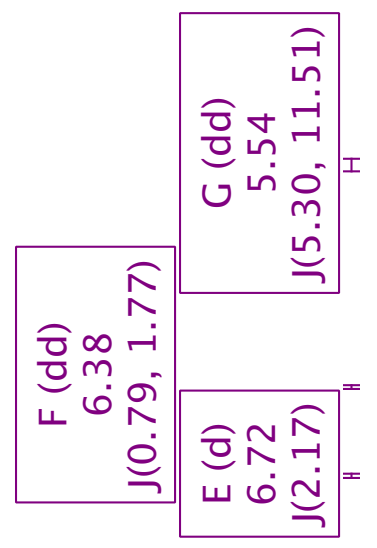

⿳ำ
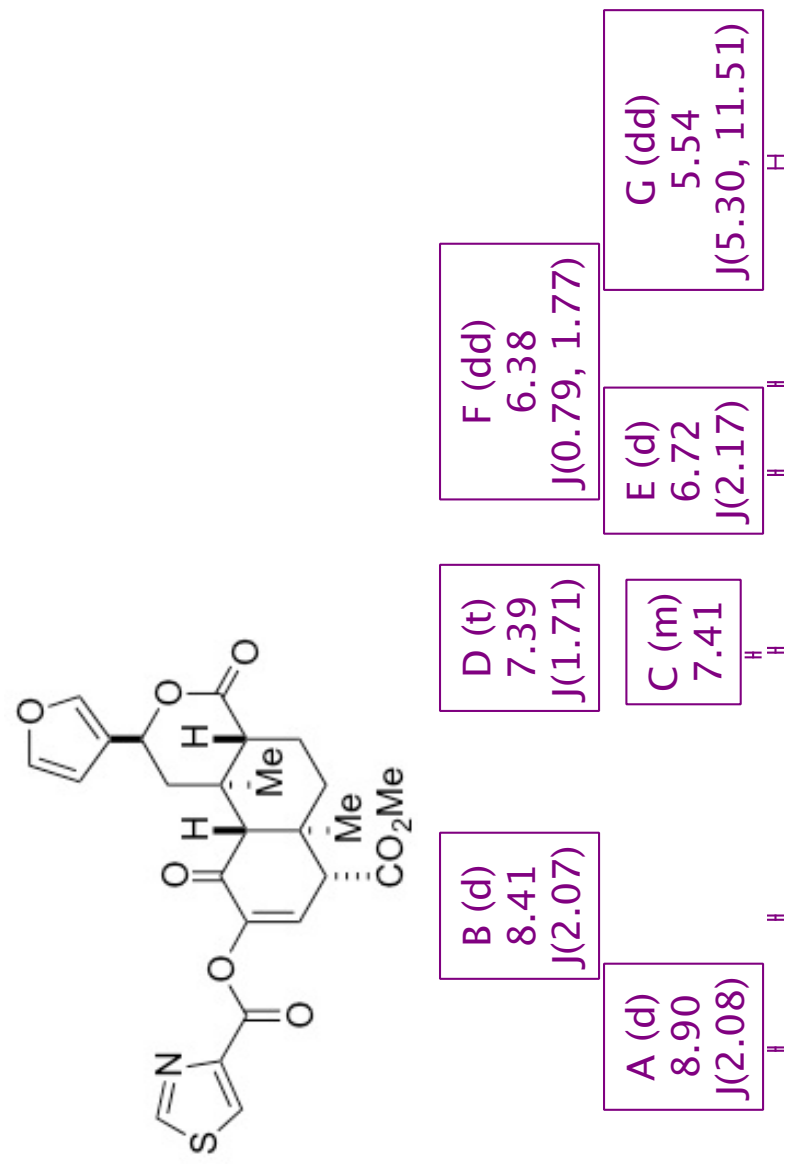


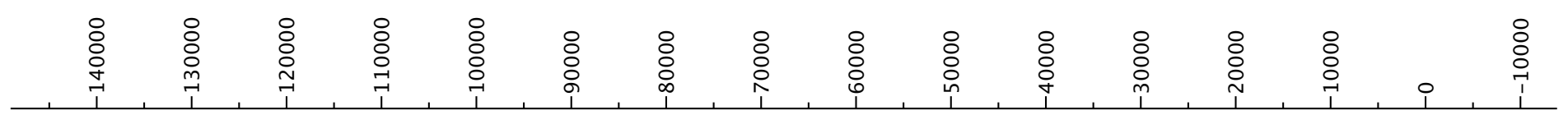

$98^{\circ} \circ \mathrm{I}$

L 8.9 I
S6. $\angle \mathrm{I}$

$\downarrow 8^{\circ} \varsigma \varepsilon-$

$\angle \nabla^{\circ} 8 \varepsilon^{-}$

น L E†T

$\varepsilon \iota^{*} \bullet{ }^{\circ}$

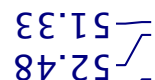

$8 \nabla^{\circ}$ 'S

$8 \varepsilon^{\circ} \varepsilon 9-$

$\angle 0^{\circ} 2 \angle-$

Z๐・80I-

$\varepsilon \nabla^{\circ} \varsigma \mathrm{L}$ ح

St.6ZI

$\rightarrow \varepsilon^{\circ} 0 \varepsilon \tau-$

$\varepsilon \varepsilon^{\circ} 6 \varepsilon \mathrm{I}$

$0 \angle \cdot \varepsilon \sqcup[\backslash$

20.StI-

$0 \mathrm{I}^{\circ} 9 \mathrm{t} \mathrm{I} 厅$

I6. $\varepsilon \mathrm{I}-$

$8 L^{\circ} 8 \mathrm{SI}-$

S ${ }^{\circ} 0 \angle \mathrm{I}$

$9 \varepsilon^{\circ}\left[\angle \tau^{-}\right.$

$\nabla S^{\circ} 06 \mathrm{I}-$ 

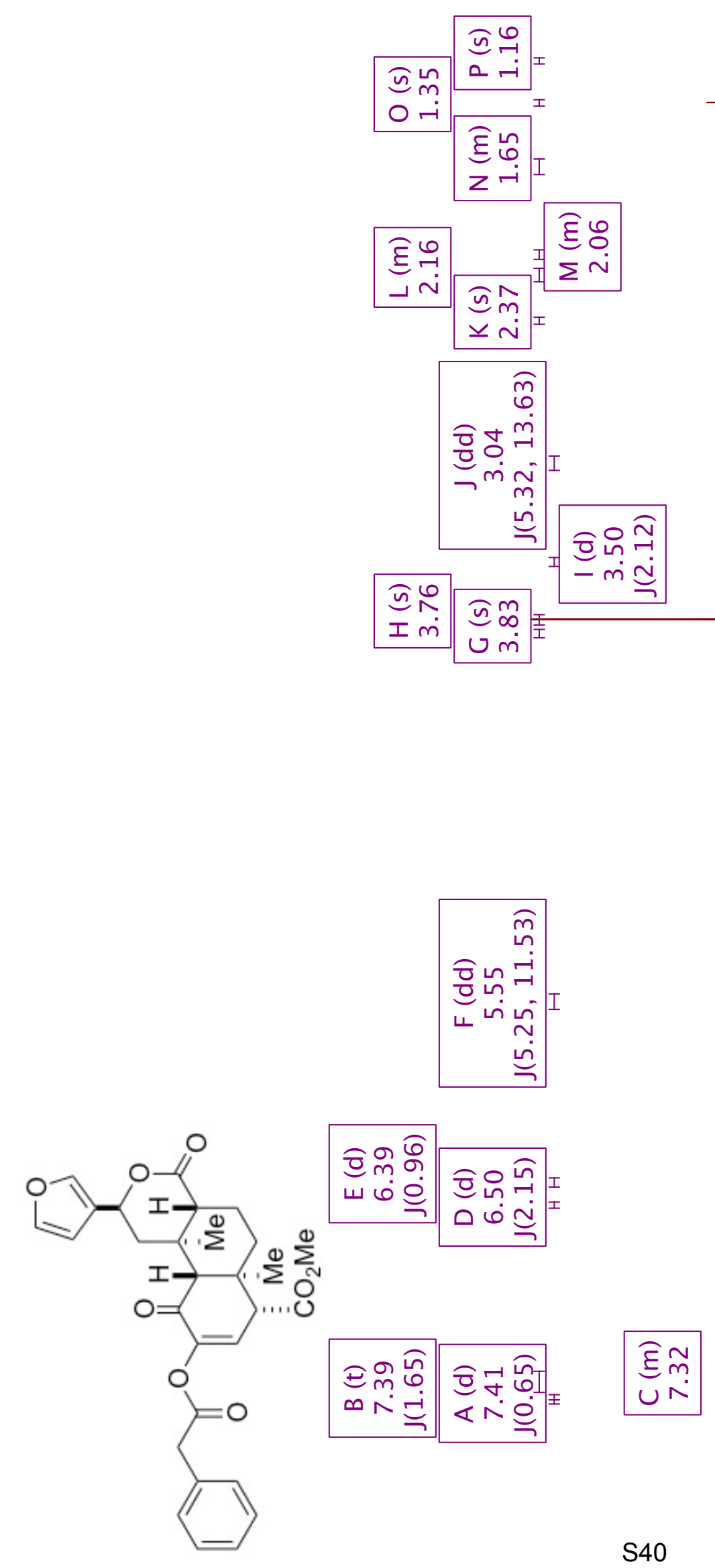

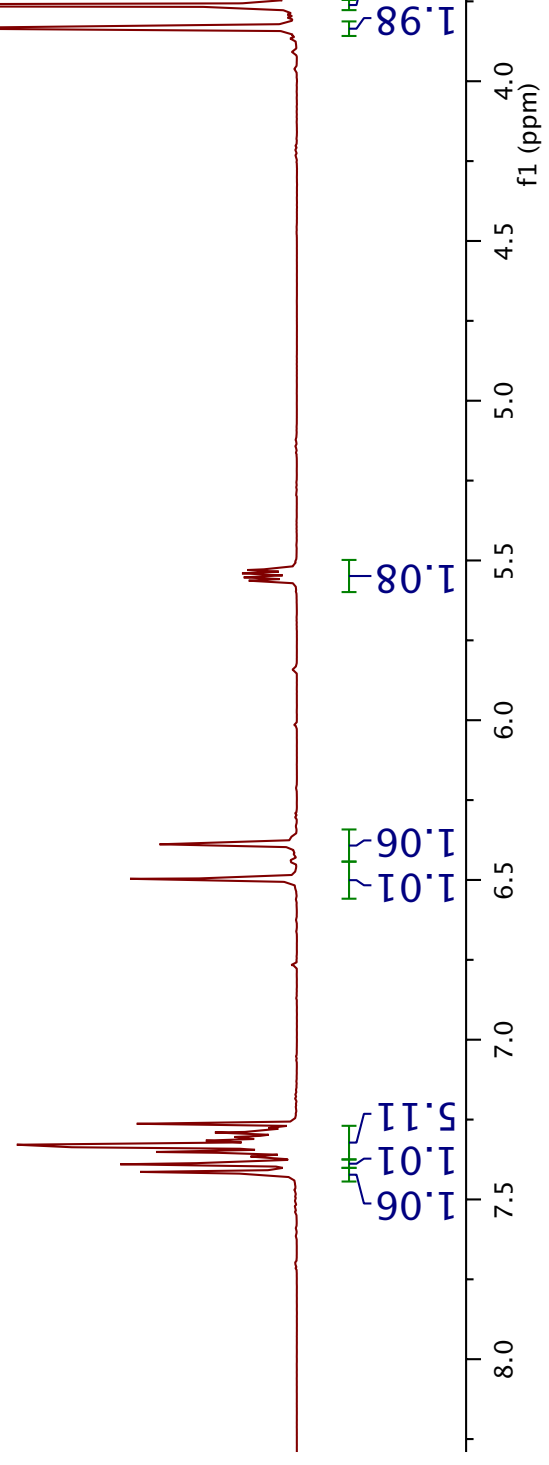


โ8.

L $\angle \cdot 9 \mathrm{I}-$

$6 L^{\circ} \varsigma \varepsilon>$

$\varsigma \varepsilon \cdot 8 \varepsilon-$

$0 \varepsilon^{\circ} 0 t^{\top}$

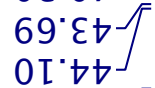

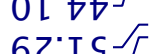

2t $25{ }^{\circ}$

$\neg \varepsilon^{\circ} 9 \varsigma$

$8 \varepsilon^{\circ} \varepsilon 9^{-}$

66. $T \angle-$

$0 S^{\circ} 80 \mathrm{I}-$

$\left.\begin{array}{l}0 t^{\circ} \varsigma Z I_{1} \\ \text { [ }{ }^{\circ} \angle Z I \\ 0 \angle L^{\circ}\end{array}\right]$

$0 \angle 8 Z \mathrm{~L}$

2๐.62I

$68.62 \mathrm{I}$

$68 \cdot 2 \varepsilon \mathrm{I}$

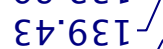

$\angle 9^{\circ} \varepsilon \nabla \tau^{\top} \digamma$

9Z'st[

s ${ }^{\circ} 69 \mathrm{~L}$
$0 \varepsilon^{\circ} 0 \angle \mathrm{L}$
$\angle \mathcal{E}^{\circ} \mathrm{L} \angle \mathrm{L}$

$2 L^{\circ} 06 \mathrm{I}-$

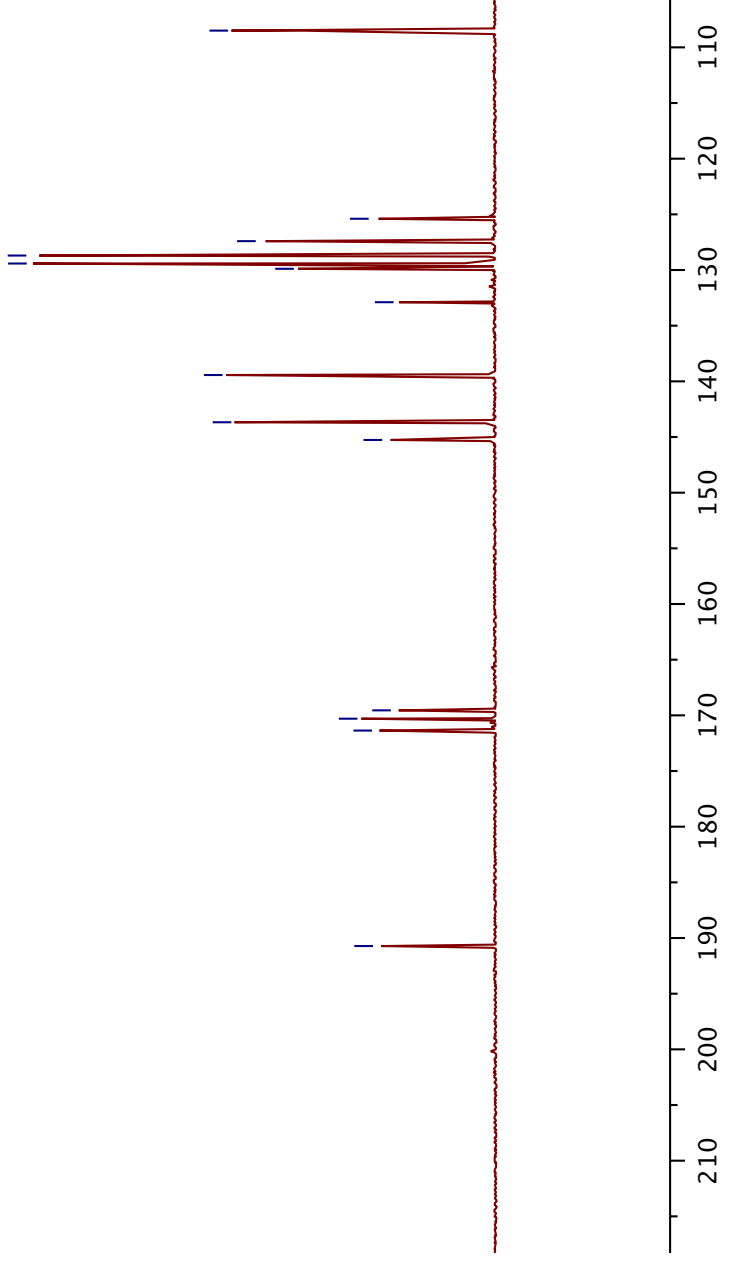




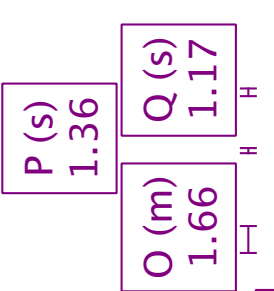

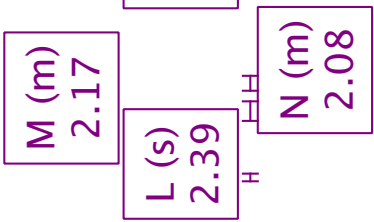

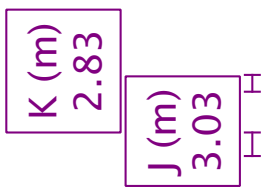

으드독
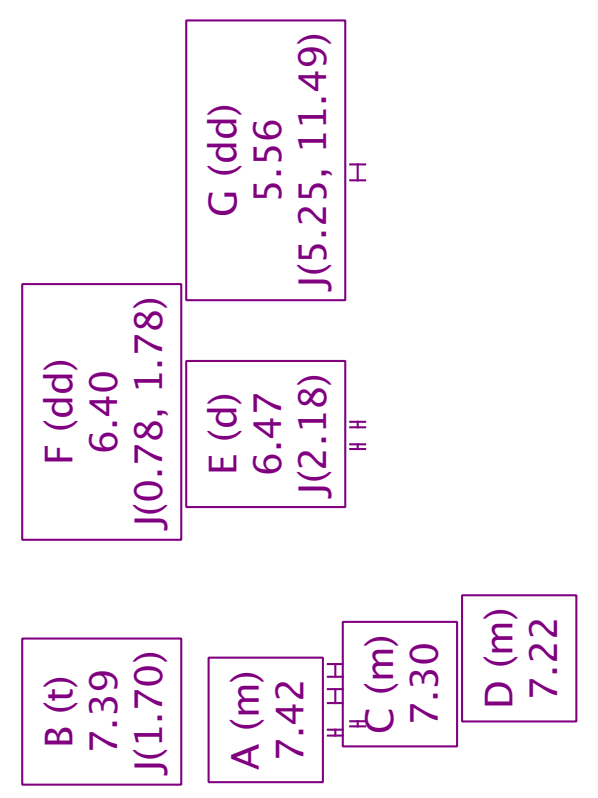
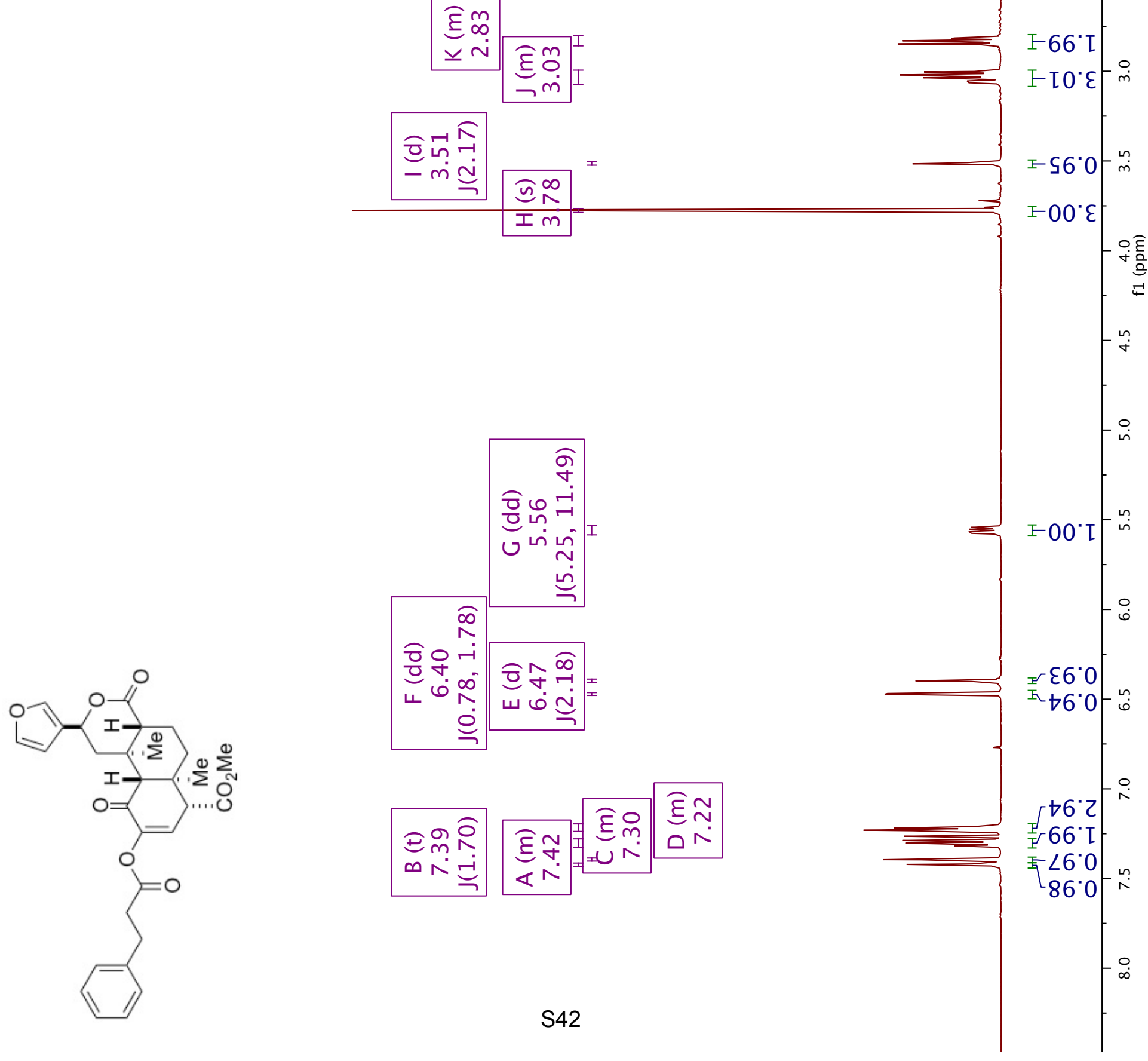
$28^{\circ} \circ \mathrm{I}$

$\varepsilon L^{\circ} 9 \mathrm{I}-$

I6 $\angle \mathrm{I}^{\top}$

69.0ع-

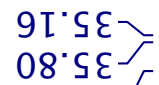

$8 \varepsilon \cdot 8 \varepsilon$

乙L'E๐

OE'IS

$9 \varepsilon^{\circ} 9 \mathrm{~s}$

ऽ $\varepsilon^{\cdot} \varepsilon 9-$

I0 $2 L-$

$8 t^{\circ} 80 \mathrm{~L}-$

乙ち.ৎZI

$\varepsilon t^{\circ} 9 Z[]$

$8 Z^{\circ} 8 Z \mathrm{~L} T$

$65^{\circ} 82 \mathrm{I}$ 厂

I ${ }^{\circ} 6 \varepsilon \mathrm{I}$

$96.6 \varepsilon[$ 工

$69^{\circ} \varepsilon \downarrow[$

$\angle I^{\circ} S \rightarrow[J$

$2 \varepsilon^{\circ} 0 \angle \mathrm{I}$
$8 \angle^{\circ} 0 \angle \mathrm{I} \mathrm{J}$
$\angle \mathcal{E}^{\circ}[\angle \mathrm{I}$

S6.06I- 


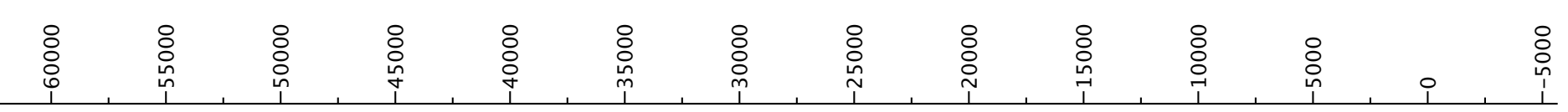
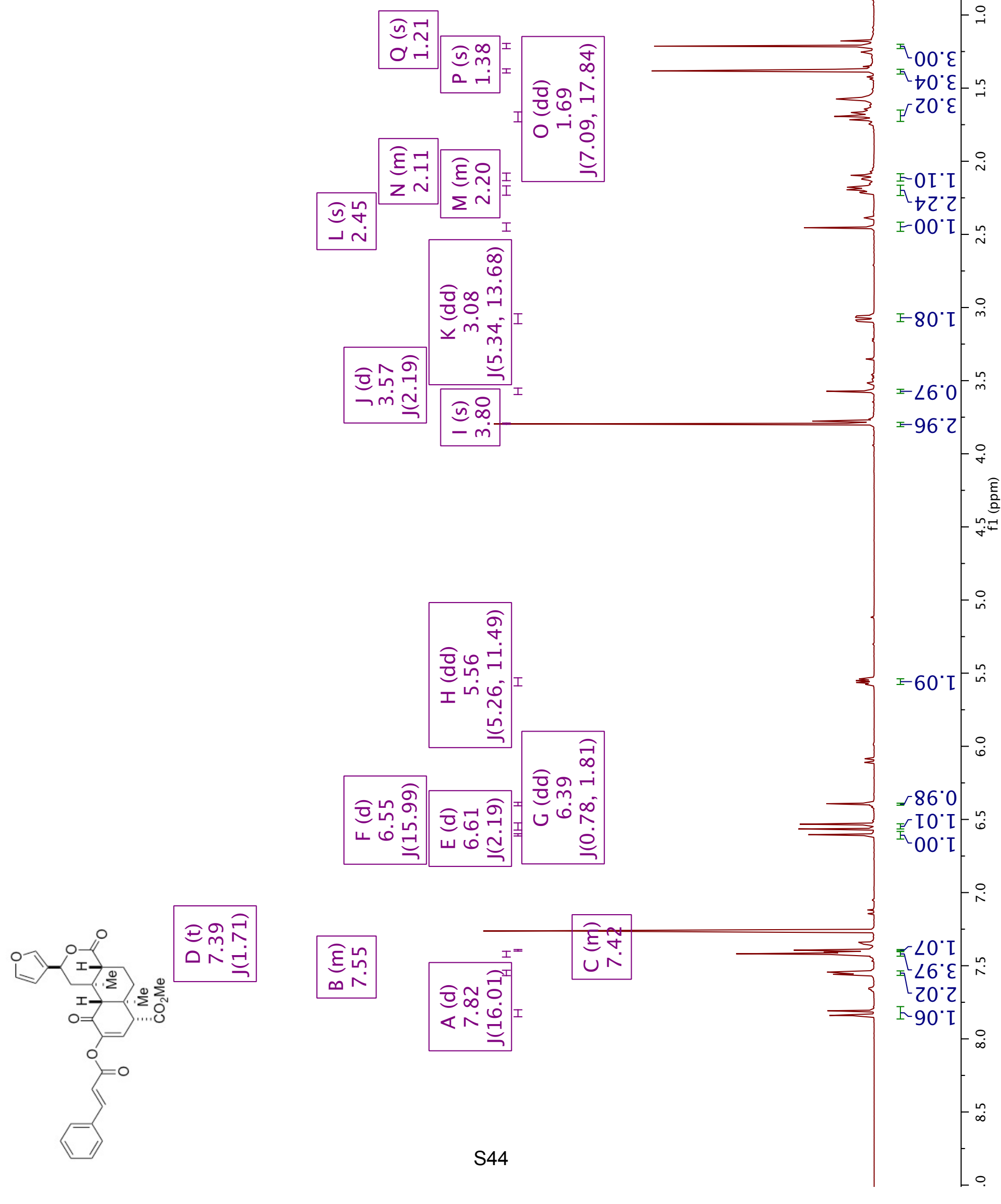
$98^{\circ} \bullet \mathrm{I}$

$6 \angle{ }^{\circ} 9 \mathrm{I}-$
S6 $\angle \mathrm{I}$

ง $8^{\circ} \varsigma \varepsilon^{-}$

$\angle t^{\circ} 8 \varepsilon^{-}$

$8 L \cdot \varepsilon \downarrow T$

$6[\circ \nabla]$

$8 \varepsilon^{\circ}$ IS

St'2S

乙๐. $\varepsilon 9$

$\angle 0^{\circ} Z \angle-$

$97^{\circ} 80 \mathrm{~L}-$

SL'SII-

$\downarrow t \cdot S Z[$

$6 \varepsilon^{\circ} 8 Z[]$

$00^{\circ} 62[$

$\varepsilon 8.62 \mathrm{~L}-\frac{-}{-}$

$\varepsilon 6^{\circ} 0 \varepsilon\left[\tau^{\circ}\right.$

乙6. $\varepsilon \varepsilon[$

$8 \varepsilon^{\circ} 6 \varepsilon[$

$89^{\circ} \varepsilon \downarrow[-5$

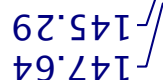

$99^{\circ} \triangleright 9 I-$

$0 t^{\circ} 0 \angle \mathrm{I}$ 工
$0 \nabla^{\circ}[\angle \mathrm{I}$ 


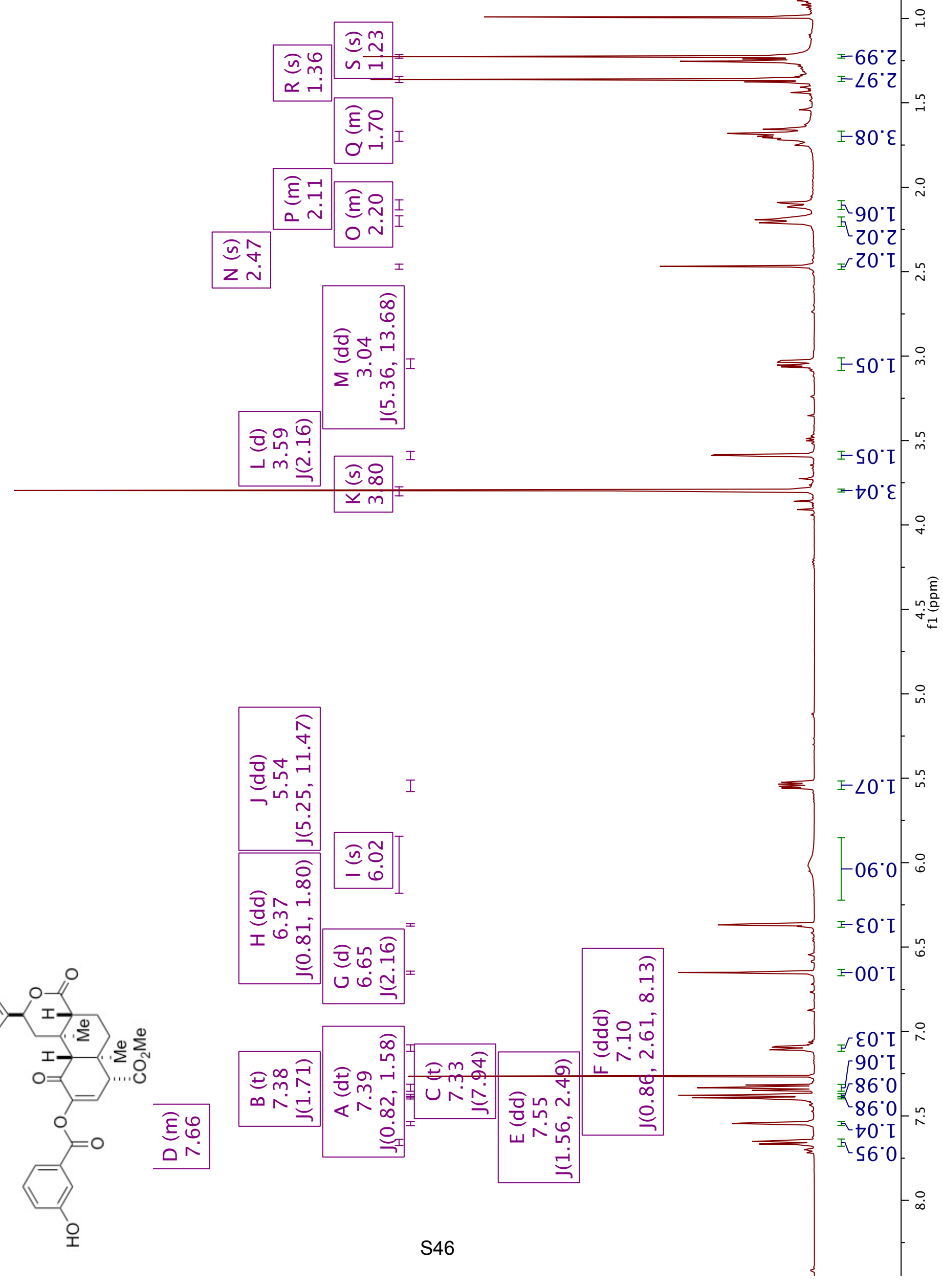




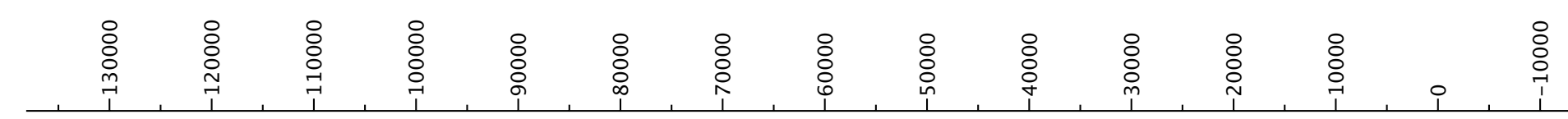

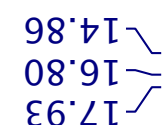

$\downarrow 8.5 \varepsilon-$

$6 \varepsilon .8 \varepsilon^{-}$

$69^{\circ} \varepsilon \nabla 厂$

$0 Z^{\circ} \bullet \nabla^{\top}$

IE' IS-

OS.2S

0t'

$6 I^{\circ} Z L-$
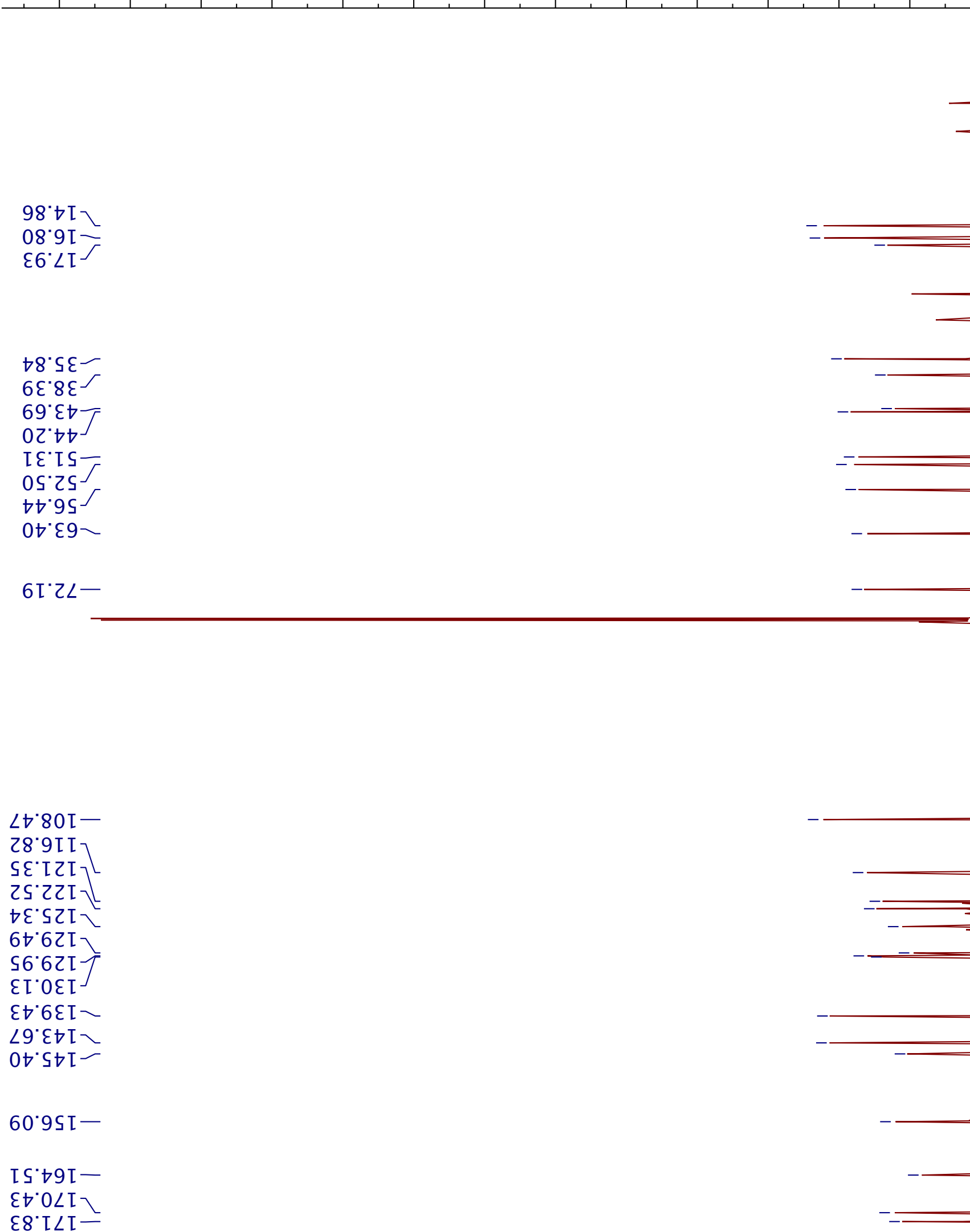

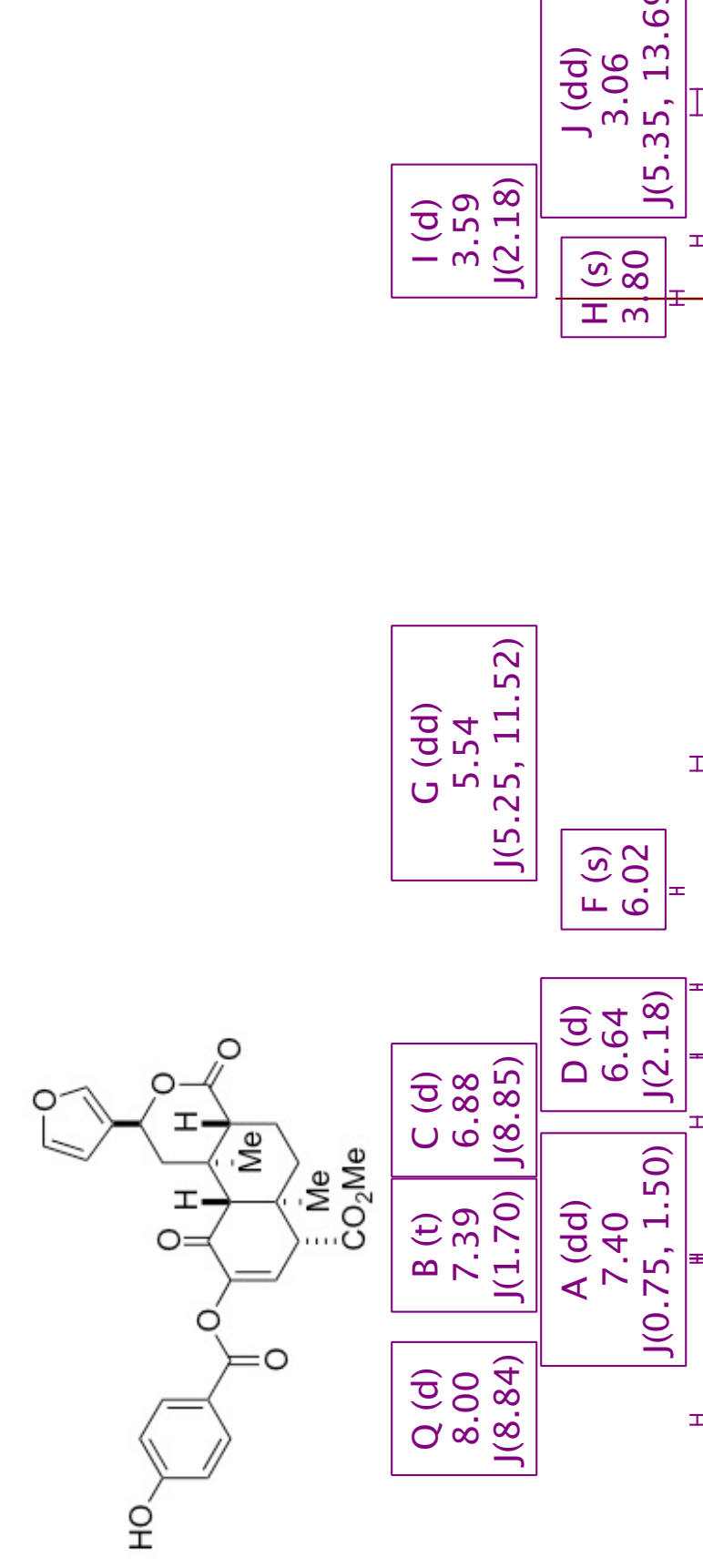
$88^{\circ} \circ \mathrm{I}$

$88^{\circ} 9 \mathrm{I}-$
$\succ 6^{\circ} \angle \mathrm{I} J$

$\varepsilon 8^{\circ} \varsigma \varepsilon-$

$\angle t^{\circ} 8 \varepsilon^{-}$

$9\llcorner\mathrm{\varepsilon} \downarrow T$

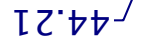

$8 \varepsilon^{\circ}$ IS

6t.25

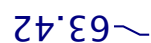

$\angle I^{\circ} Z \angle-$

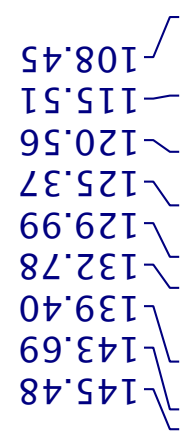

28.09I-

$\downarrow \varepsilon^{\circ} \dagger 9 \mathrm{I}-$

ZS. $0<\mathrm{L}$ 乙

99. $\mathrm{L} \angle \mathrm{I}$ 

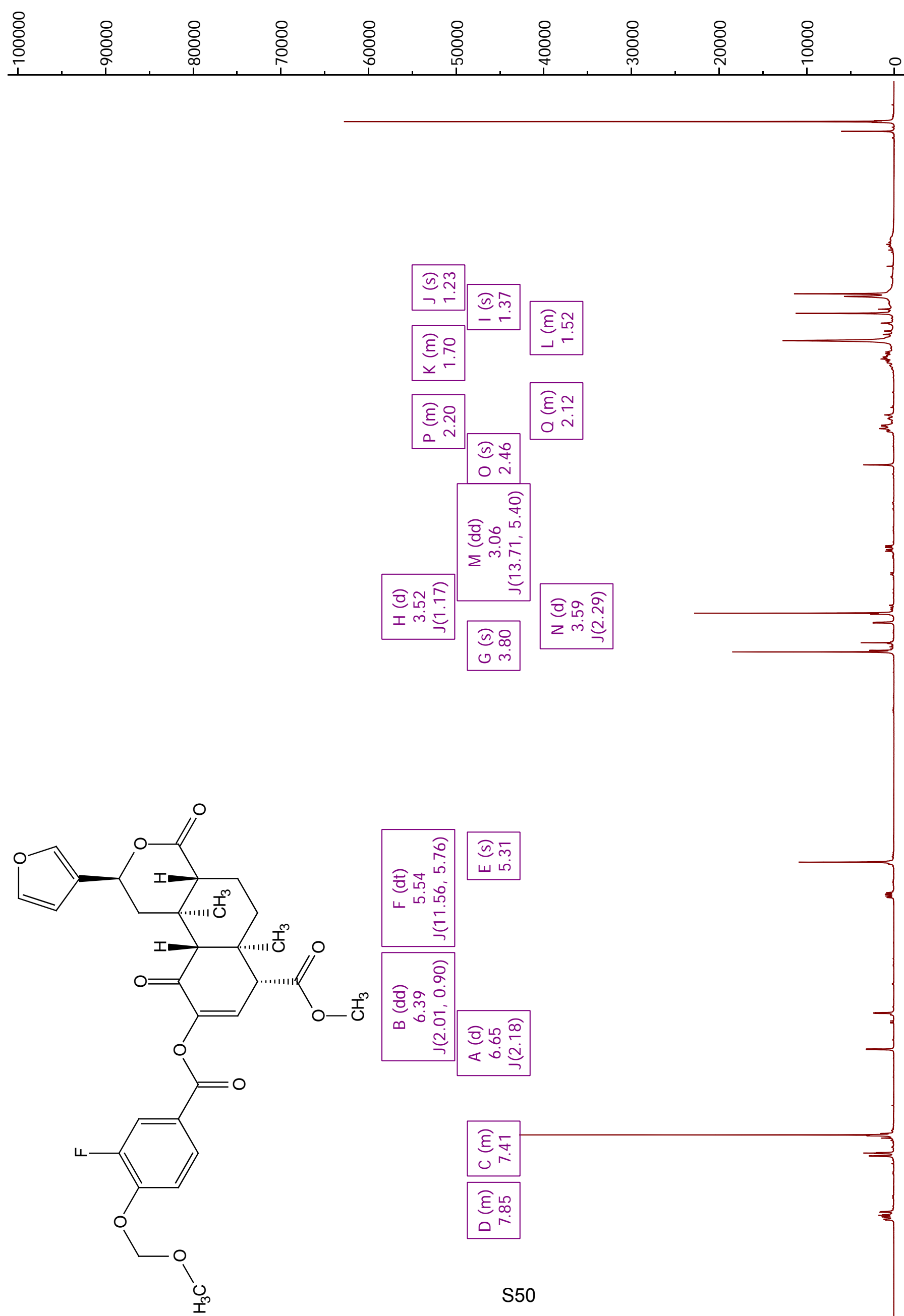


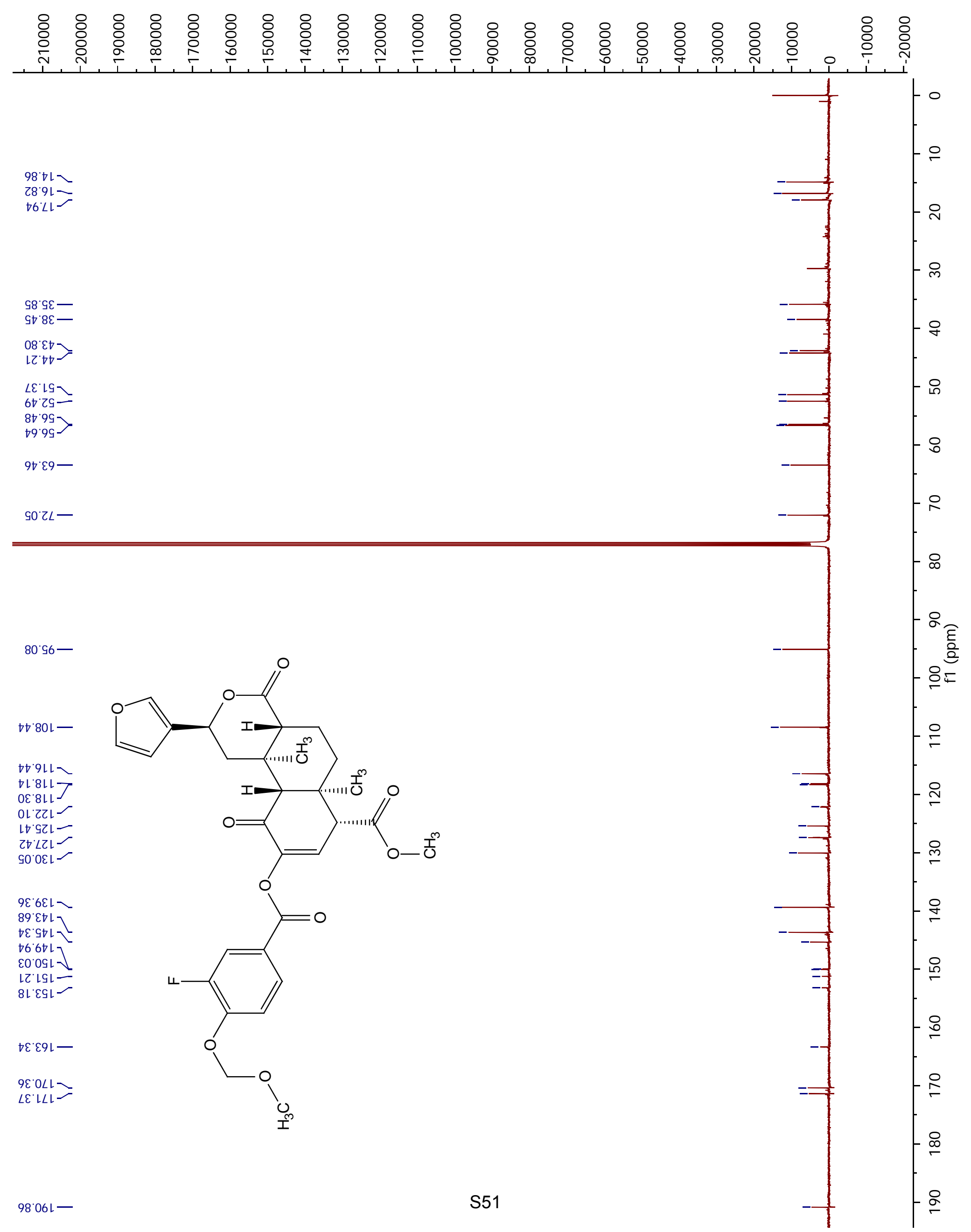




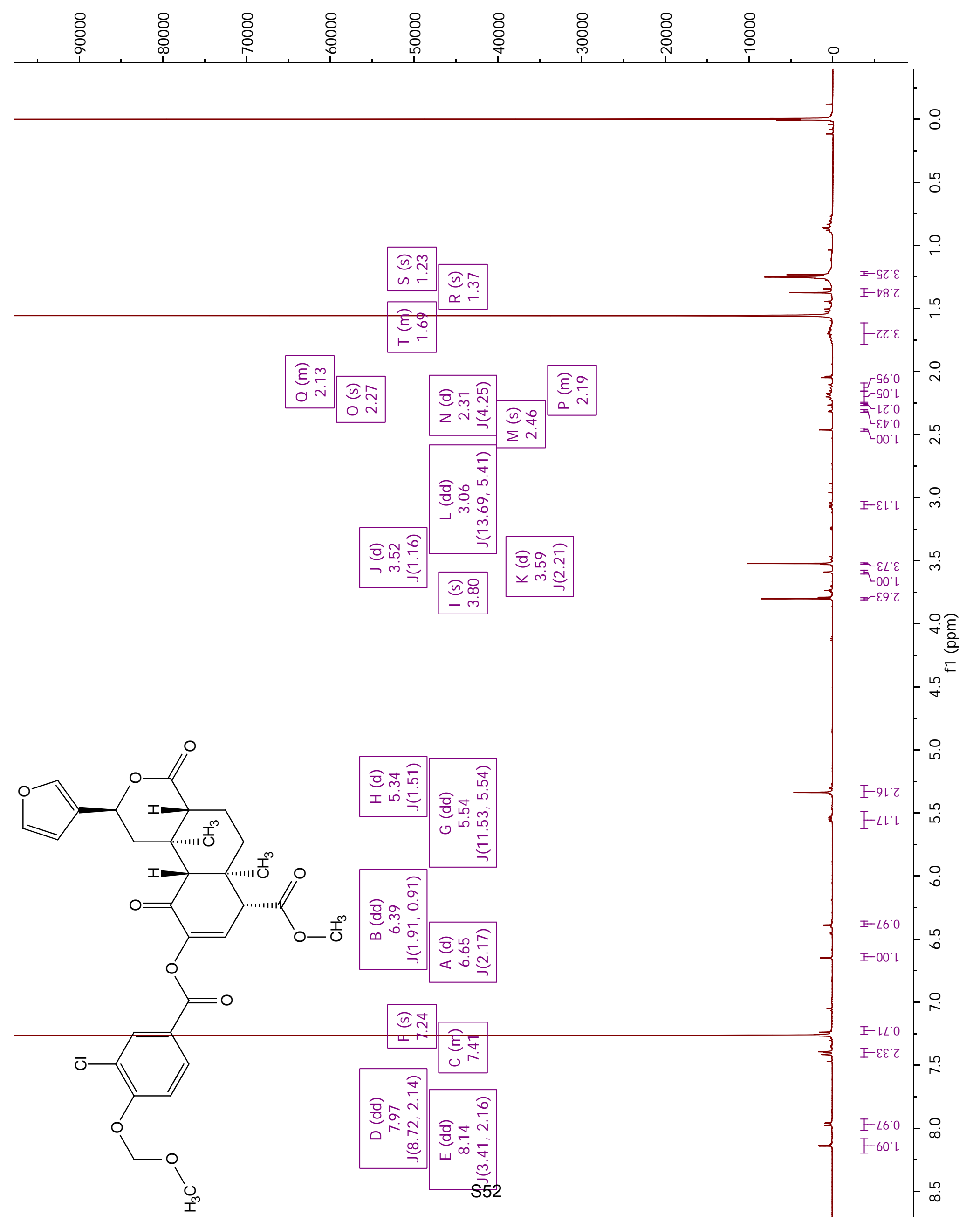




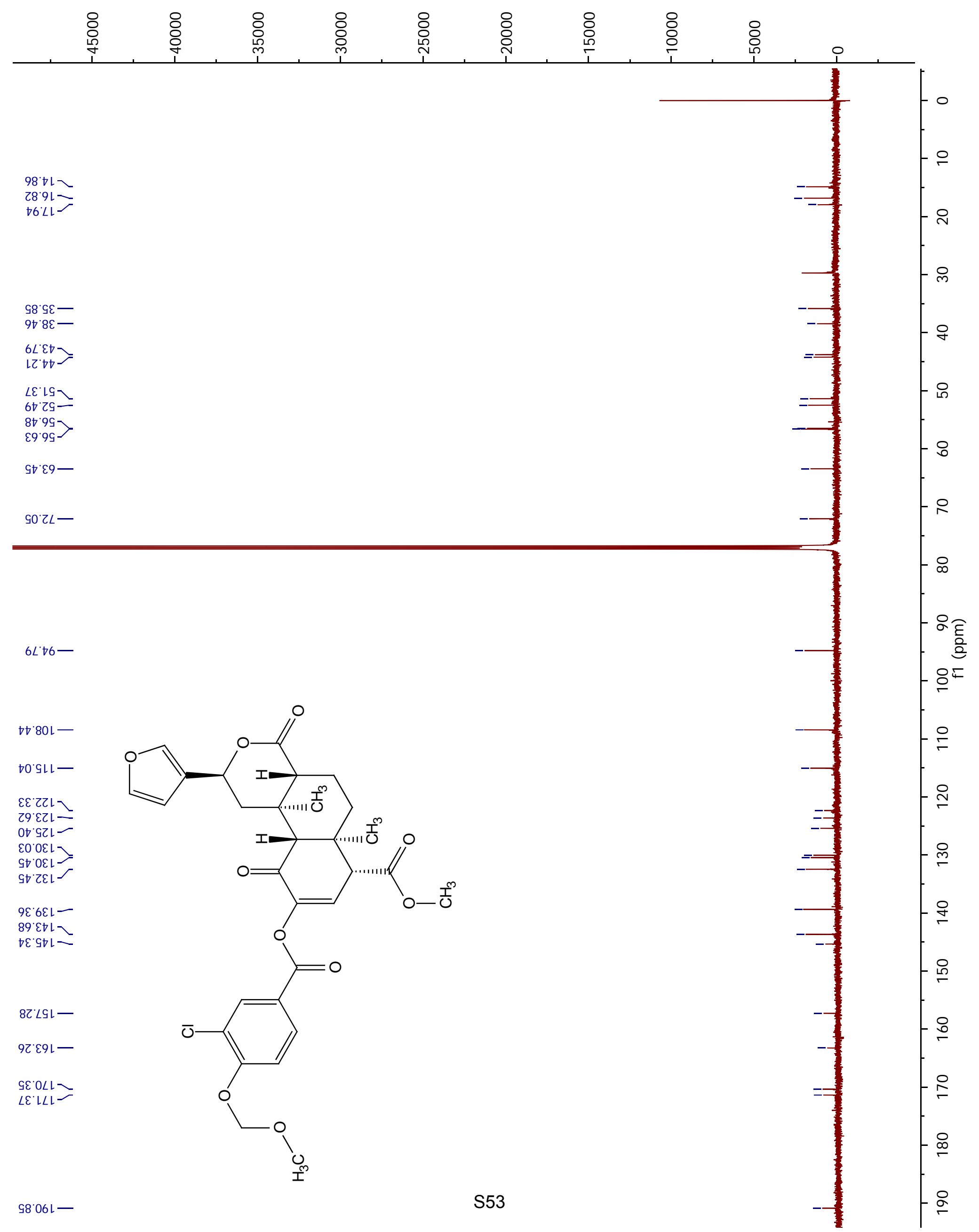




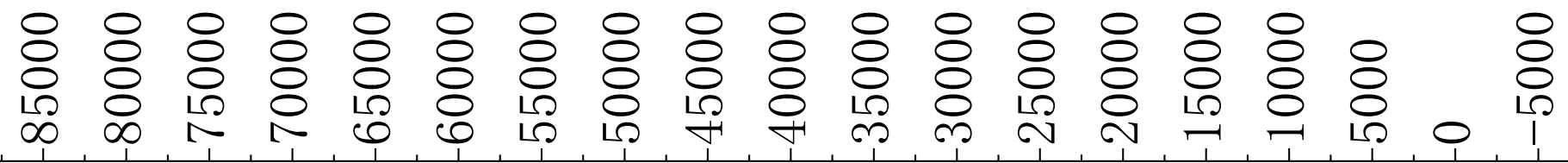

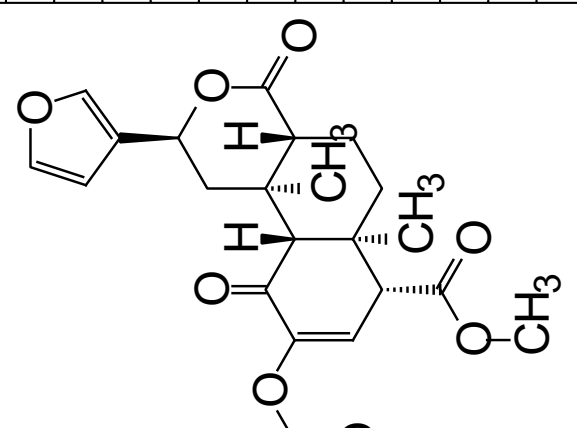<smiles>CC(=O)c1ccc(O)c(Br)c1</smiles>

O্লি

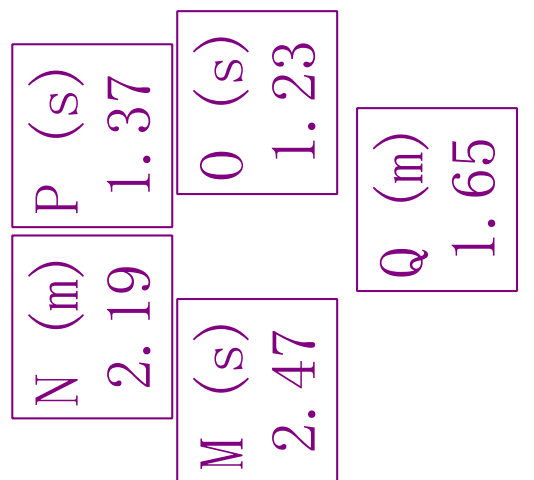

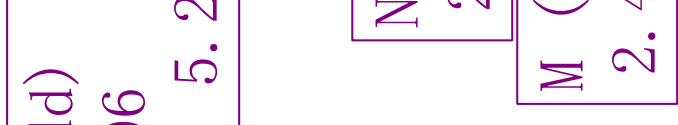

ठ

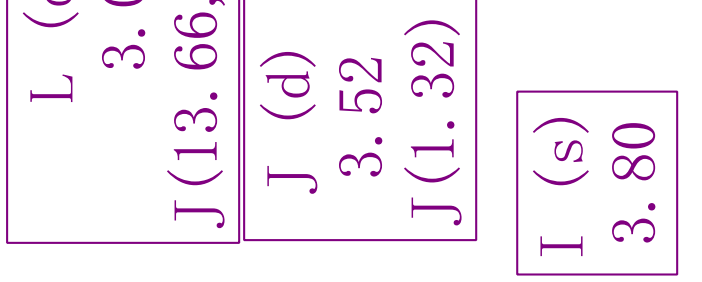
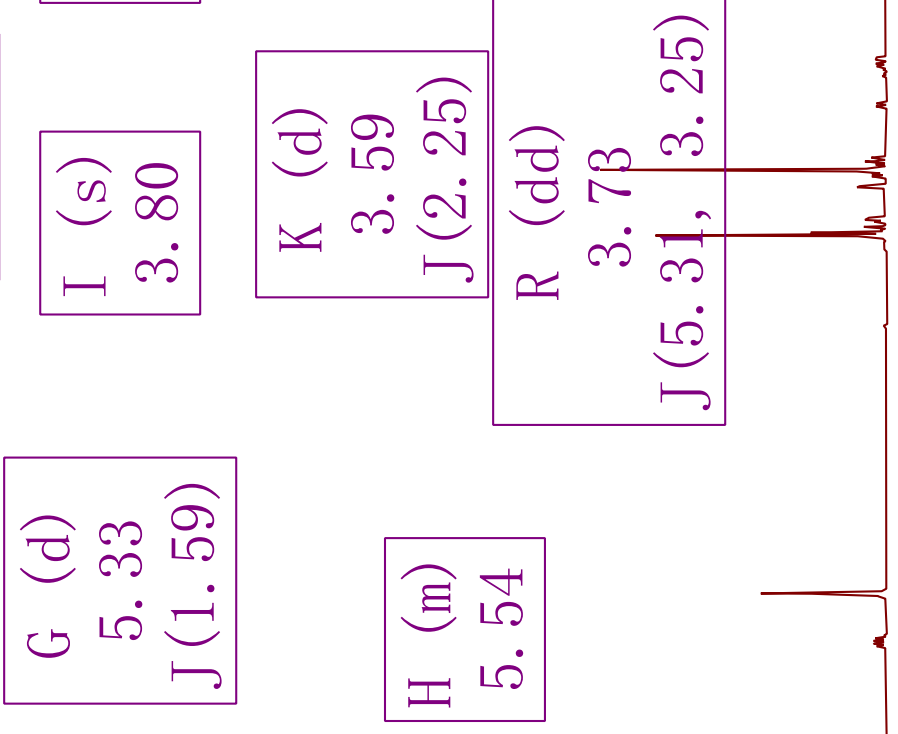

ठृ

II $\dot{0}$

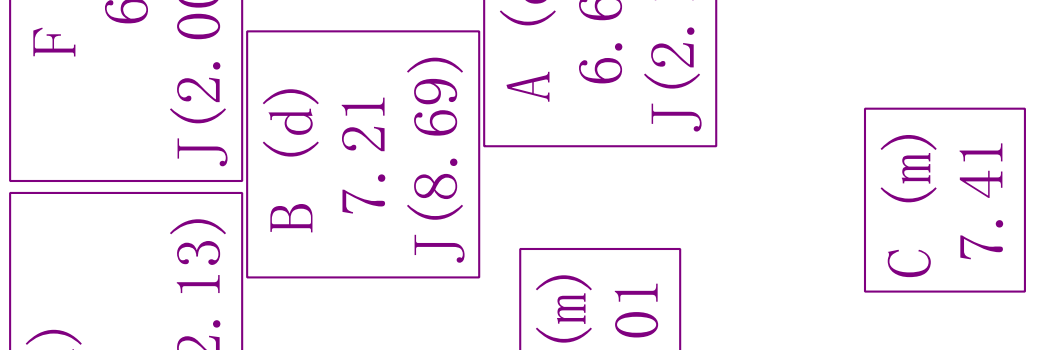

ठ $\rightarrow \dot{m}$ I $\infty$ ๙

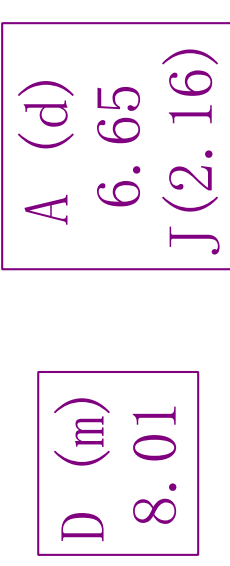

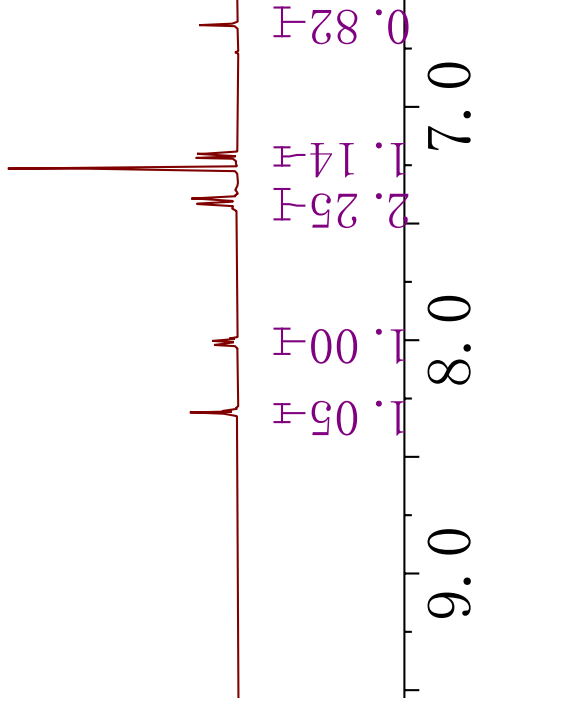




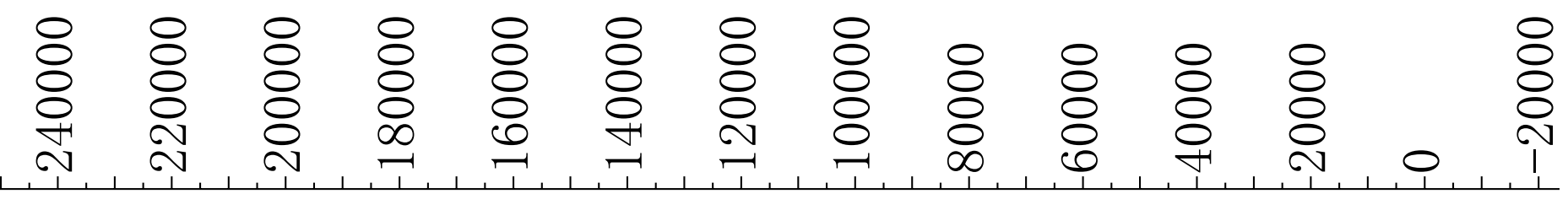

$98^{\circ}$ 万几

$\mathrm{I} 80^{\circ} \mathrm{I}$
$76{ }^{\circ} \mathrm{LI}$

$\left.78^{\circ} 9 \varepsilon\right]$

$97 \cdot 8 \varepsilon$ ר

$82^{\circ} \varepsilon \nabla 7$

I ${ }^{\circ} \nabla^{\jmath}$

GE $[\mathrm{I} \overline{\mathrm{T}}$

$87 \cdot 29^{5}$

$27 \cdot 99^{\top}$

$\varepsilon 9 \cdot 99^{\circ}$

$\varepsilon 7 \cdot \varepsilon 9$

$90{ }^{\circ} \mathrm{LL}^{\top}$

GL・ $76-$

七七 80 [ ]

$79^{\circ} \mathrm{ZII}$

$82^{\circ}$ 万I I

ZL ZZ [ ]

0万 $98 I^{2}$

$700^{\circ} 0 \varepsilon[\mathrm{I}$

LI IEI-

LG $98[-$

$9 \varepsilon \cdot 6 \varepsilon I^{\circ}$

$\angle 9{ }^{\circ} \mathrm{et} /$

E\& $\mathrm{st}$ [

G I.89 I

I I 89 I

GE $0 L$ I

LE ILI -

78 06 I-

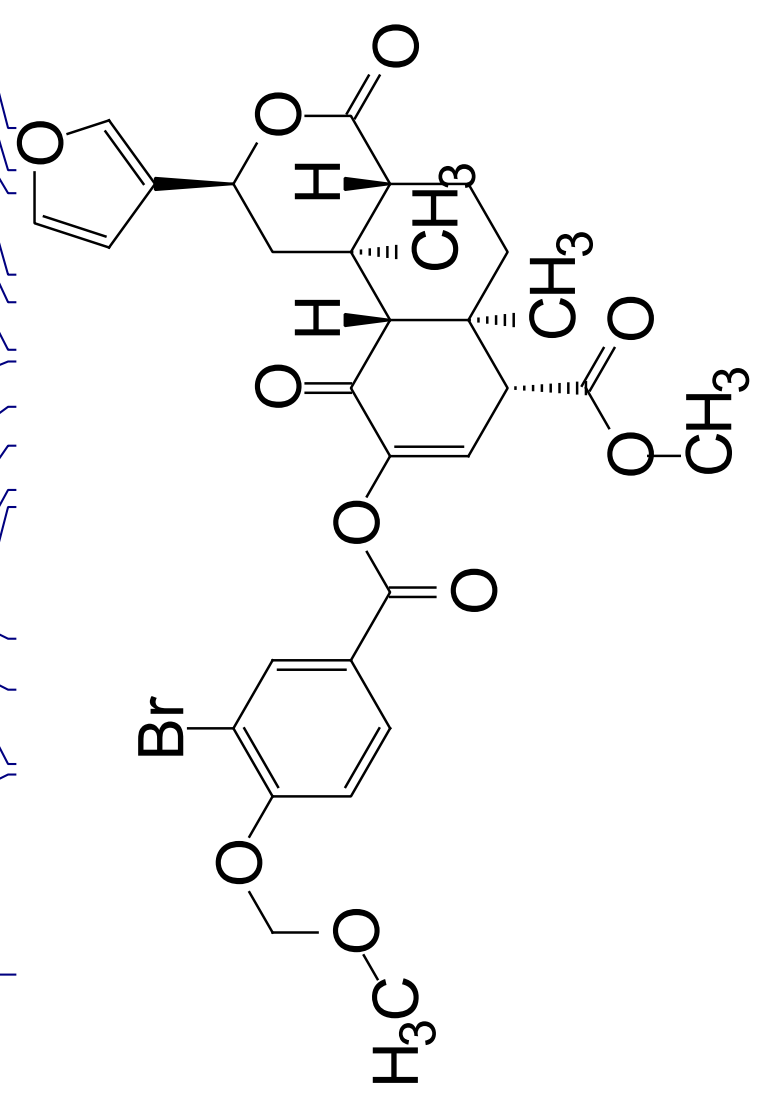




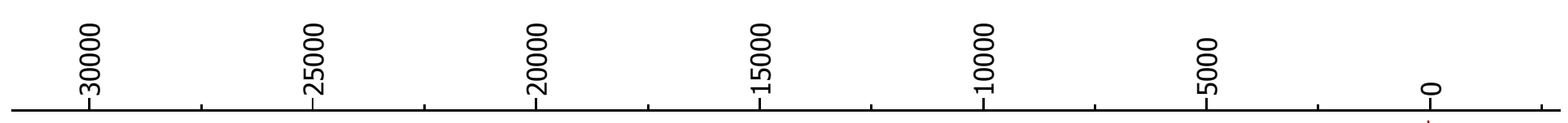
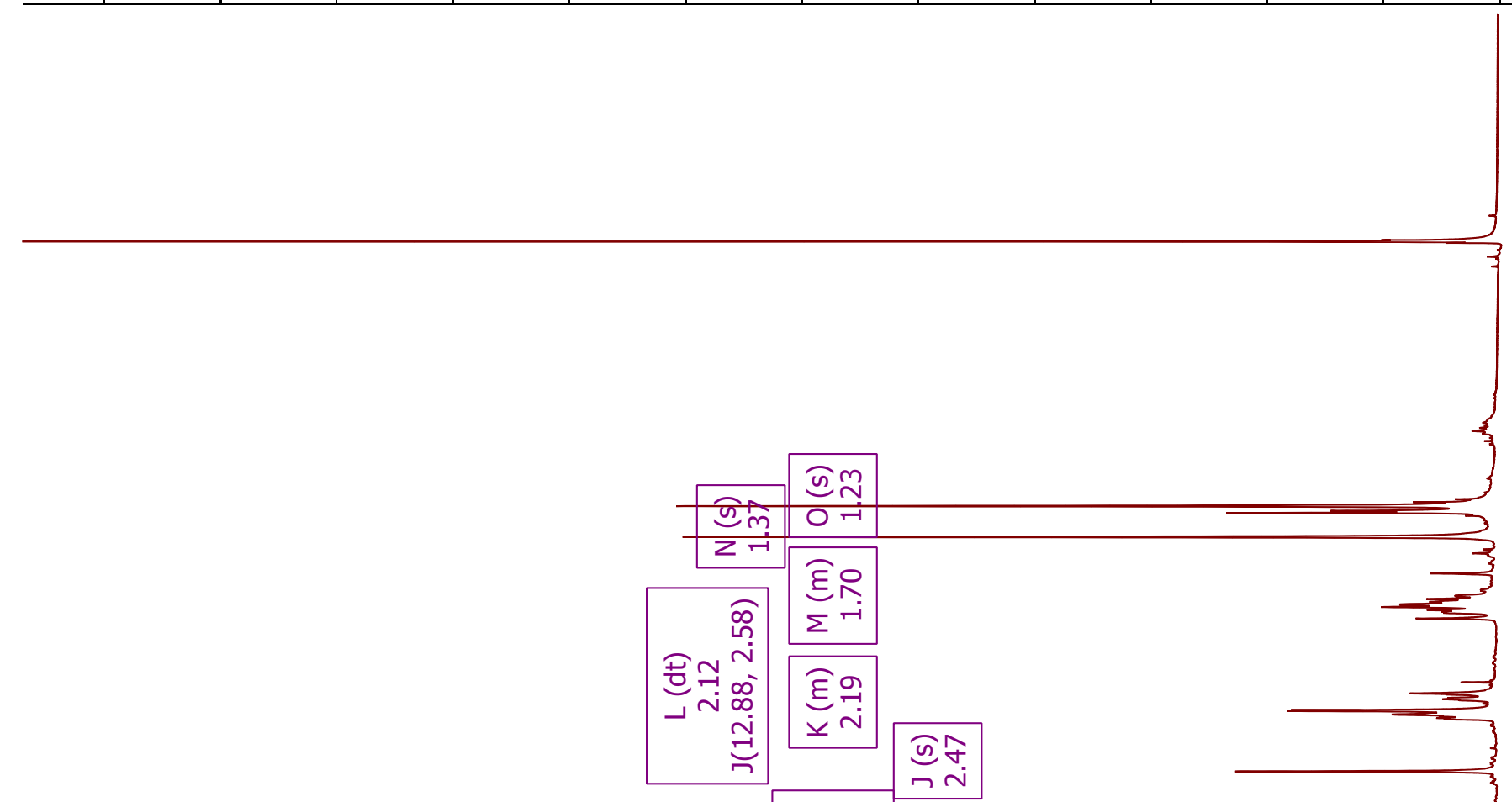

ฮิ์
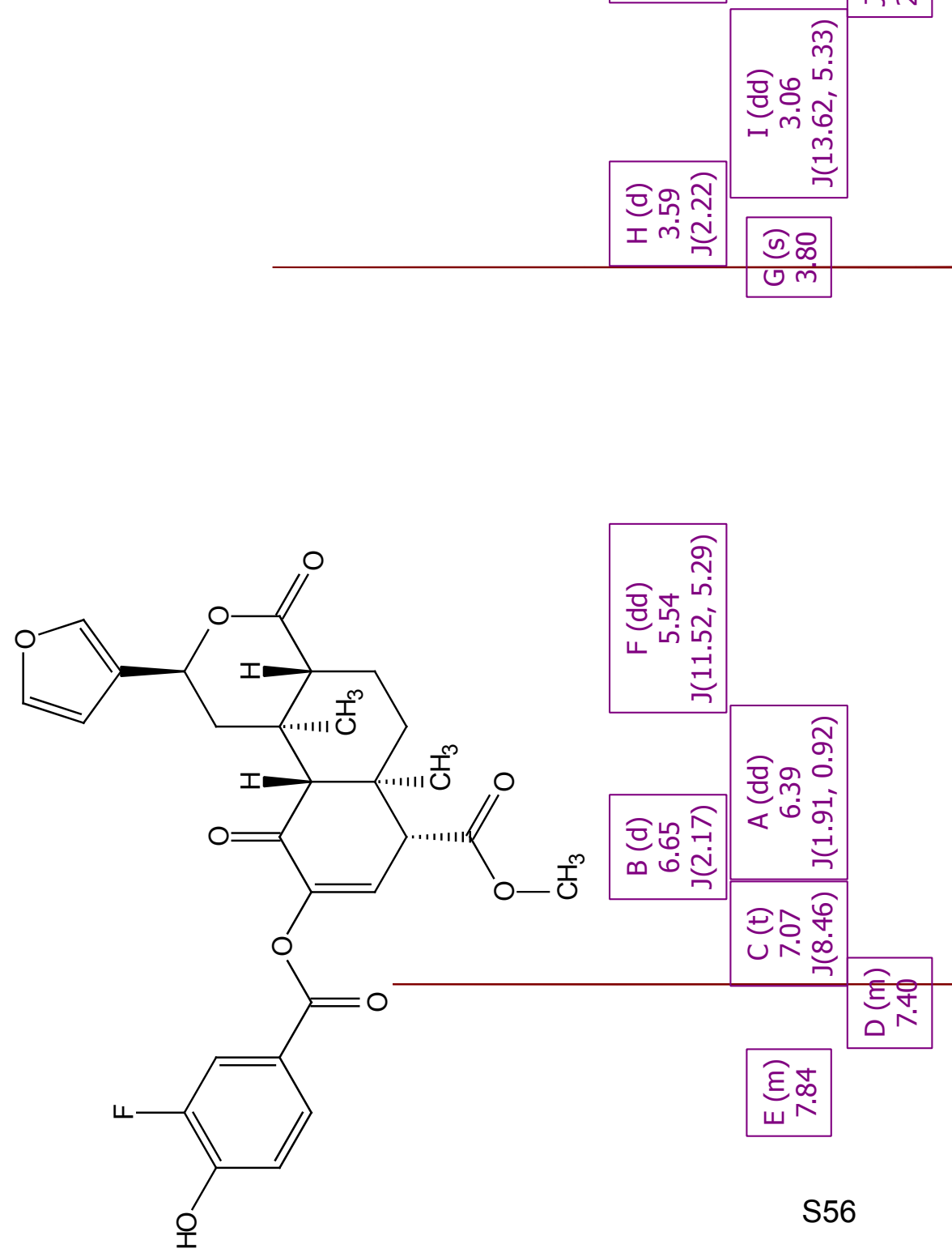

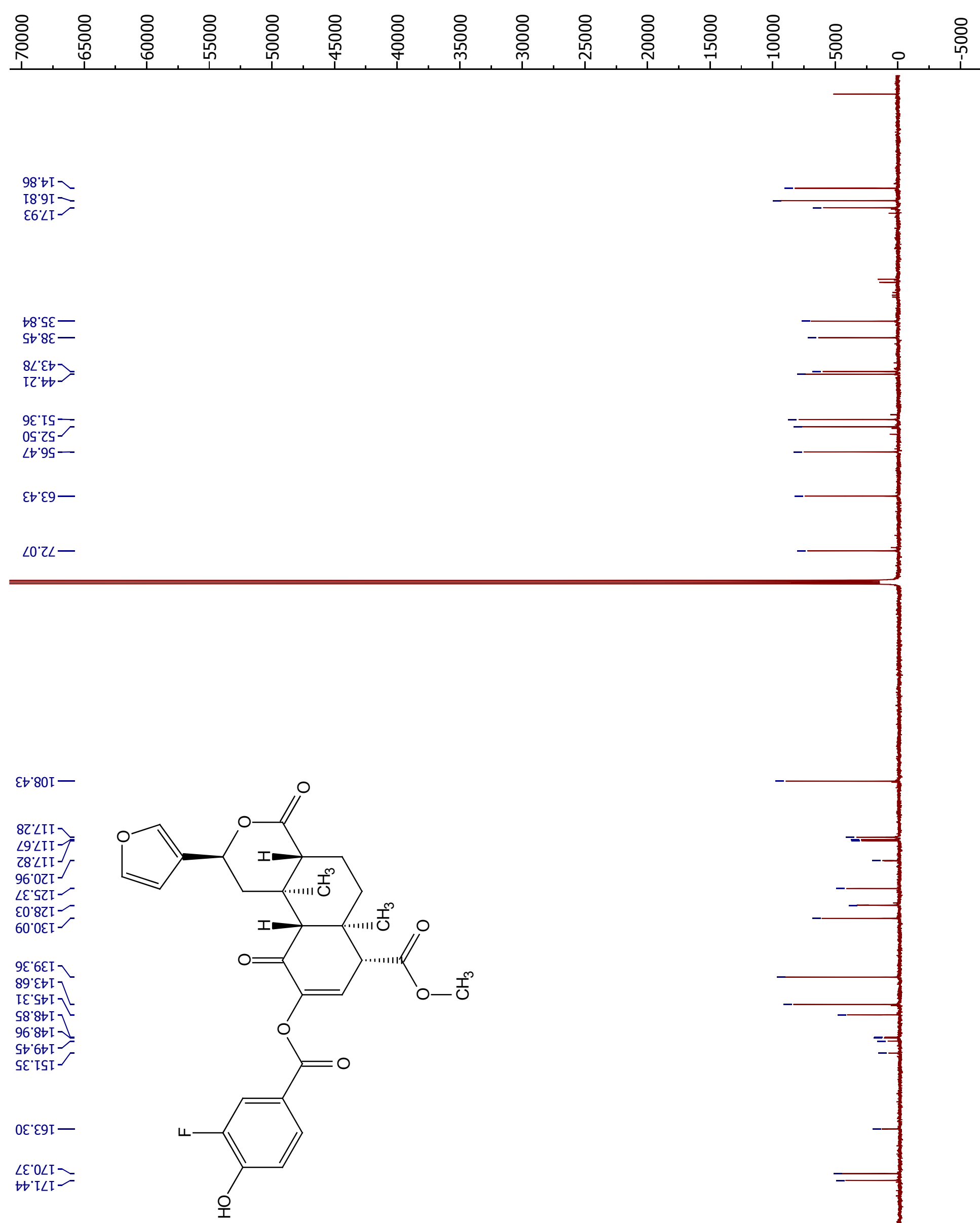


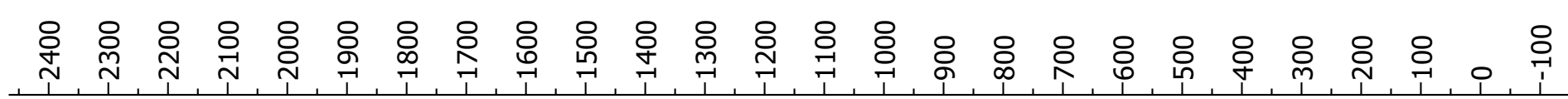

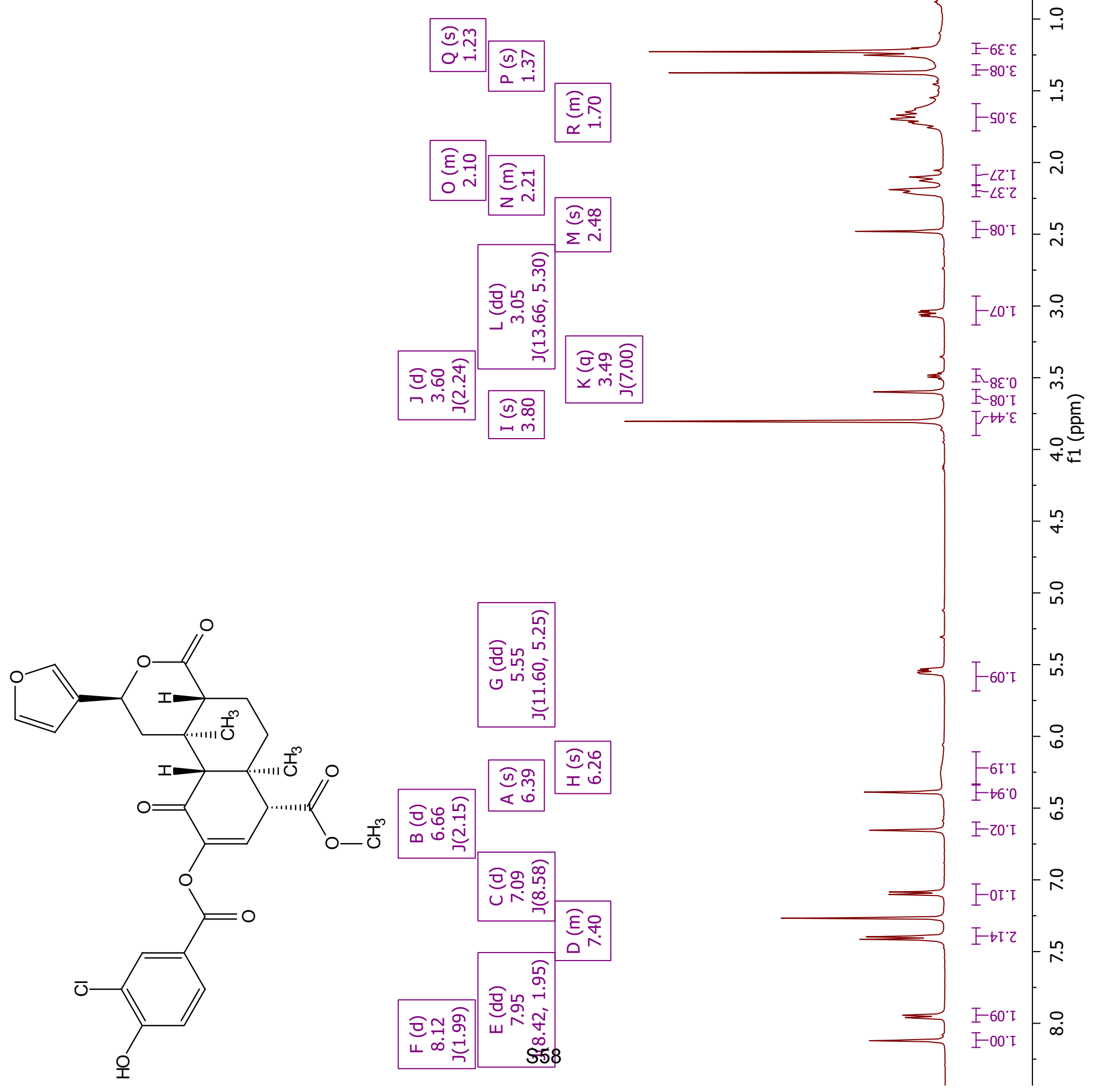




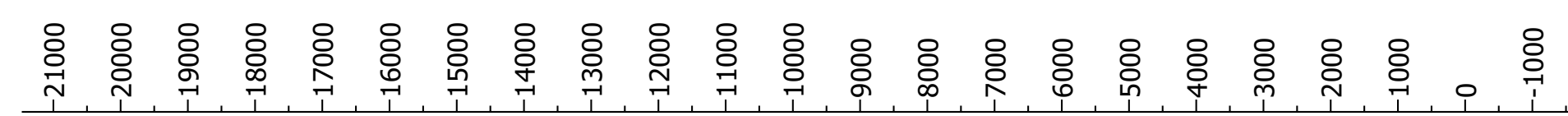

$\angle 8^{\circ} \downarrow \mathrm{I} \backslash$
$98^{\circ} 9 \mathrm{I}$

$98^{\circ} 9 \mathrm{I}-$
$\mathrm{6} \cdot \mathrm{LI}$

$\varepsilon 8 \cdot \varsigma \varepsilon-$

St. $8 \varepsilon-$

$\varepsilon L^{\prime} \varepsilon b \backslash$

9ع'IS-

$\angle S^{\circ} Z S^{-}$

๑६'६9-

OI'ZL-
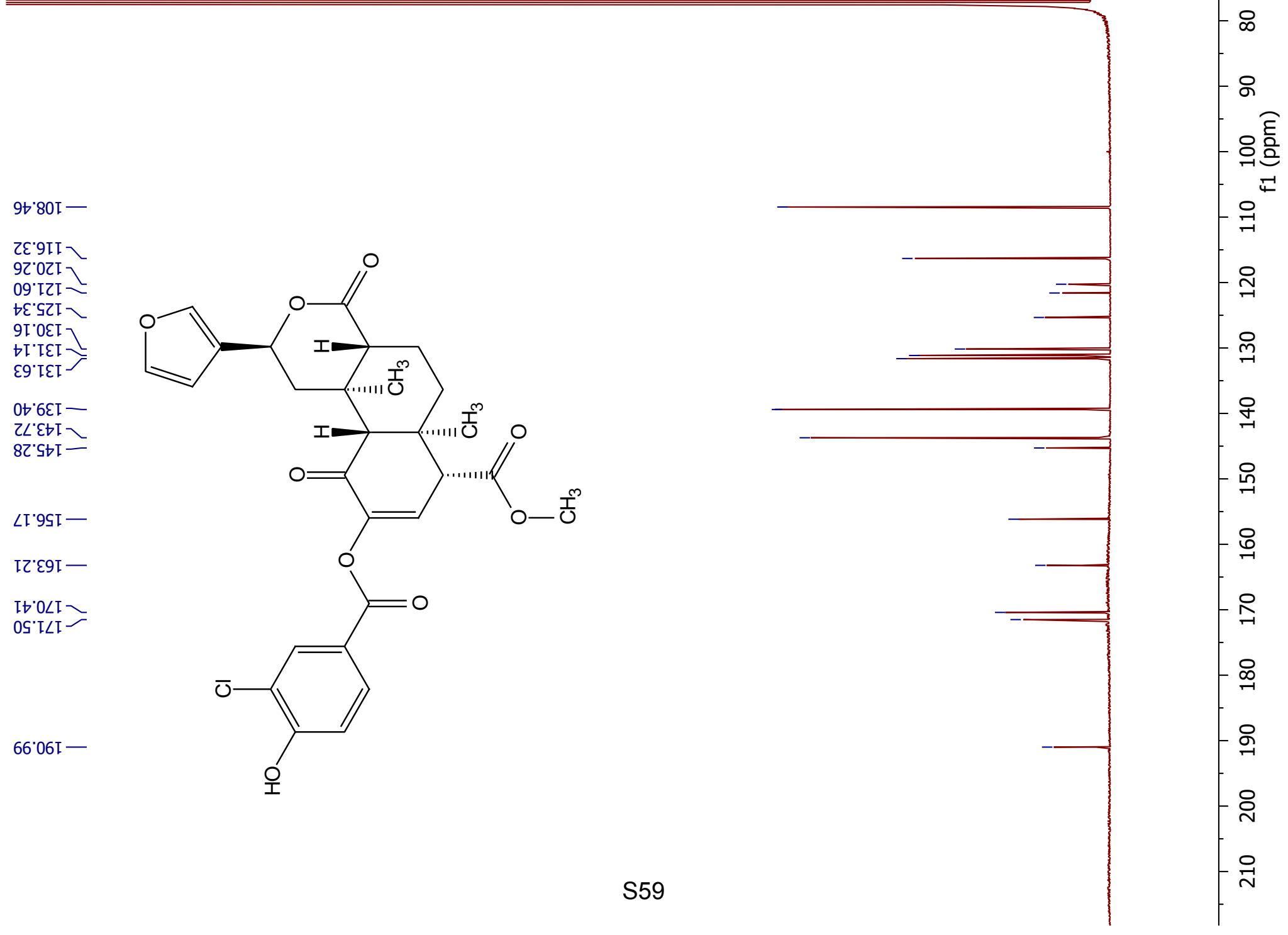


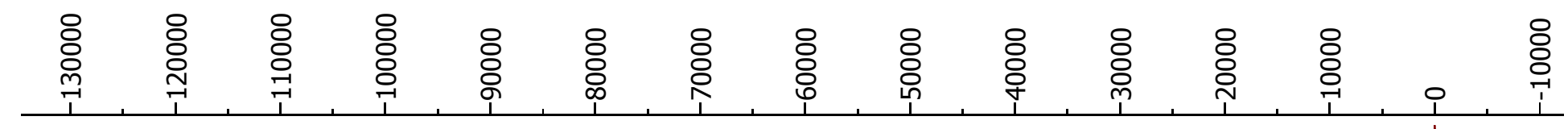
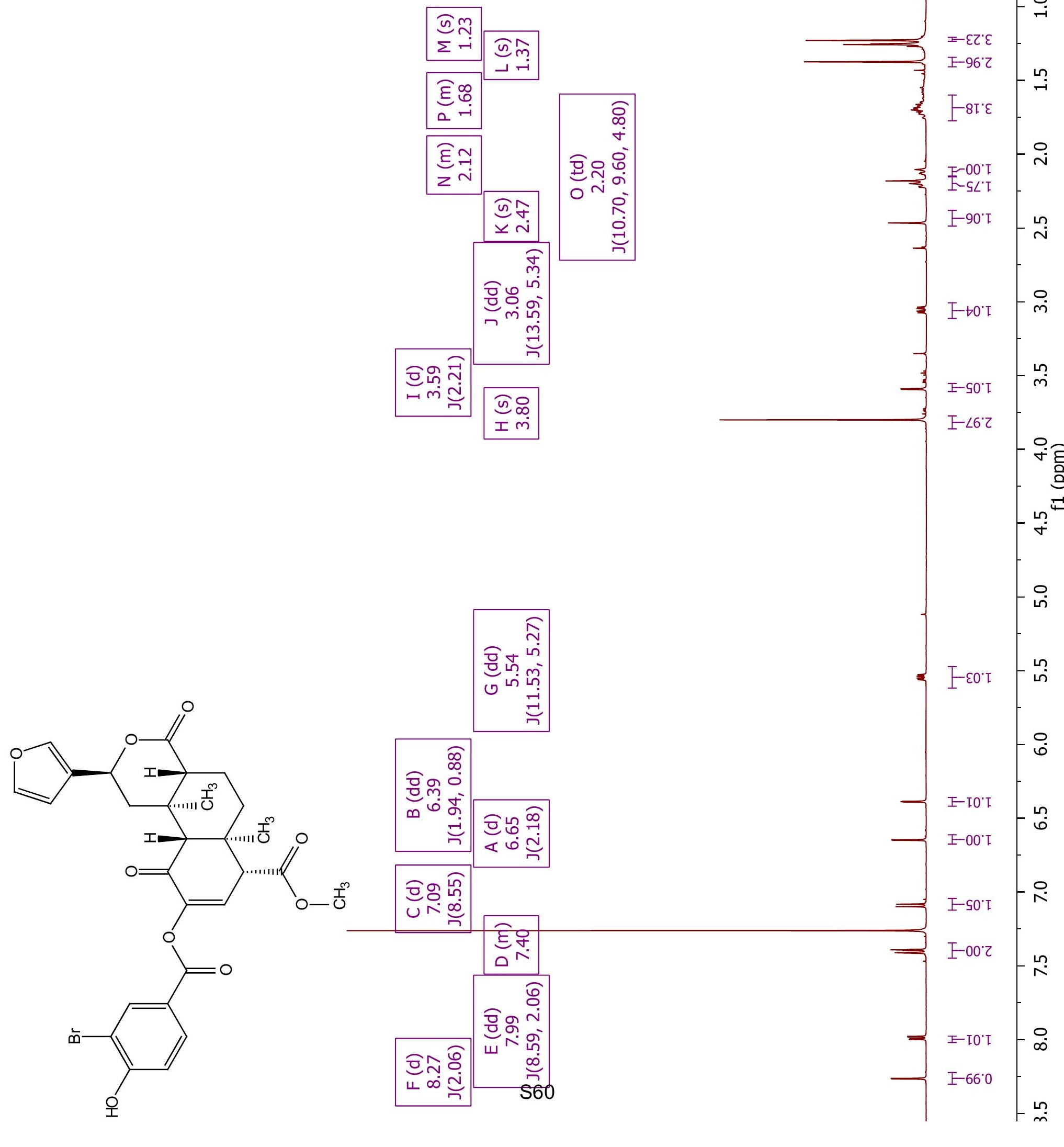


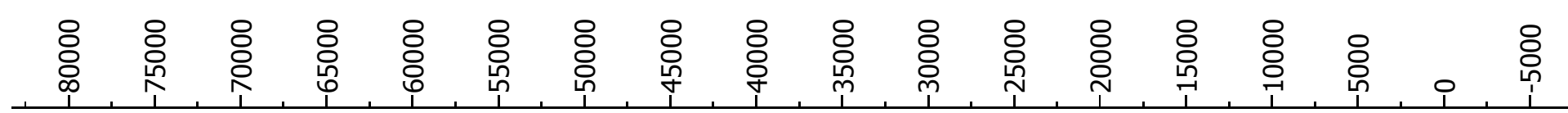

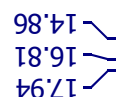

†8'ऽ -

$9 t^{\prime} 8 \varepsilon-$

6L'E七】

$9 \varepsilon \cdot T S-$

6t'2s

$\angle t^{\prime} 9 \mathrm{~S}$ -

$\varepsilon t^{\prime} \varepsilon 9-$

เO'ZL-
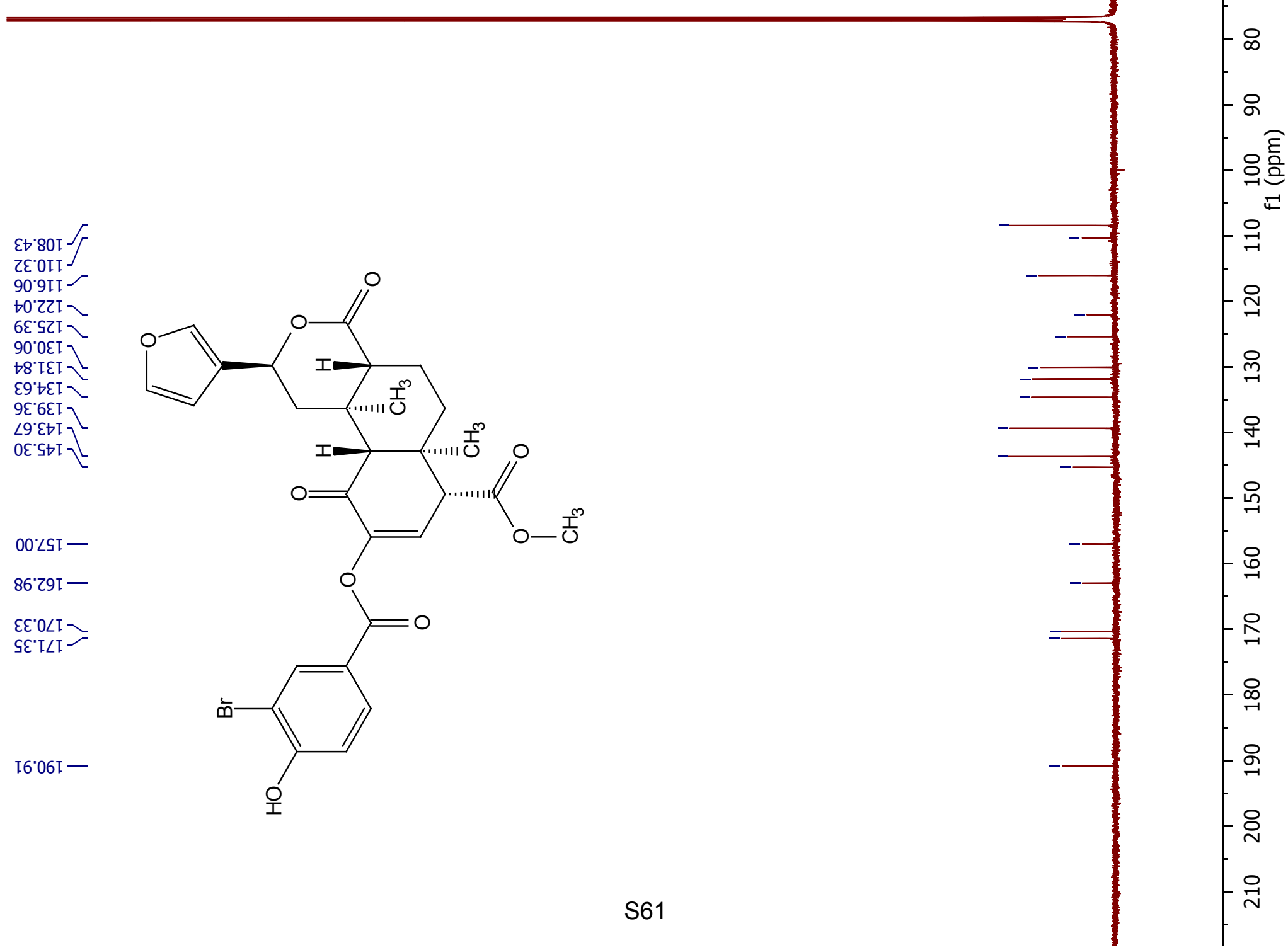


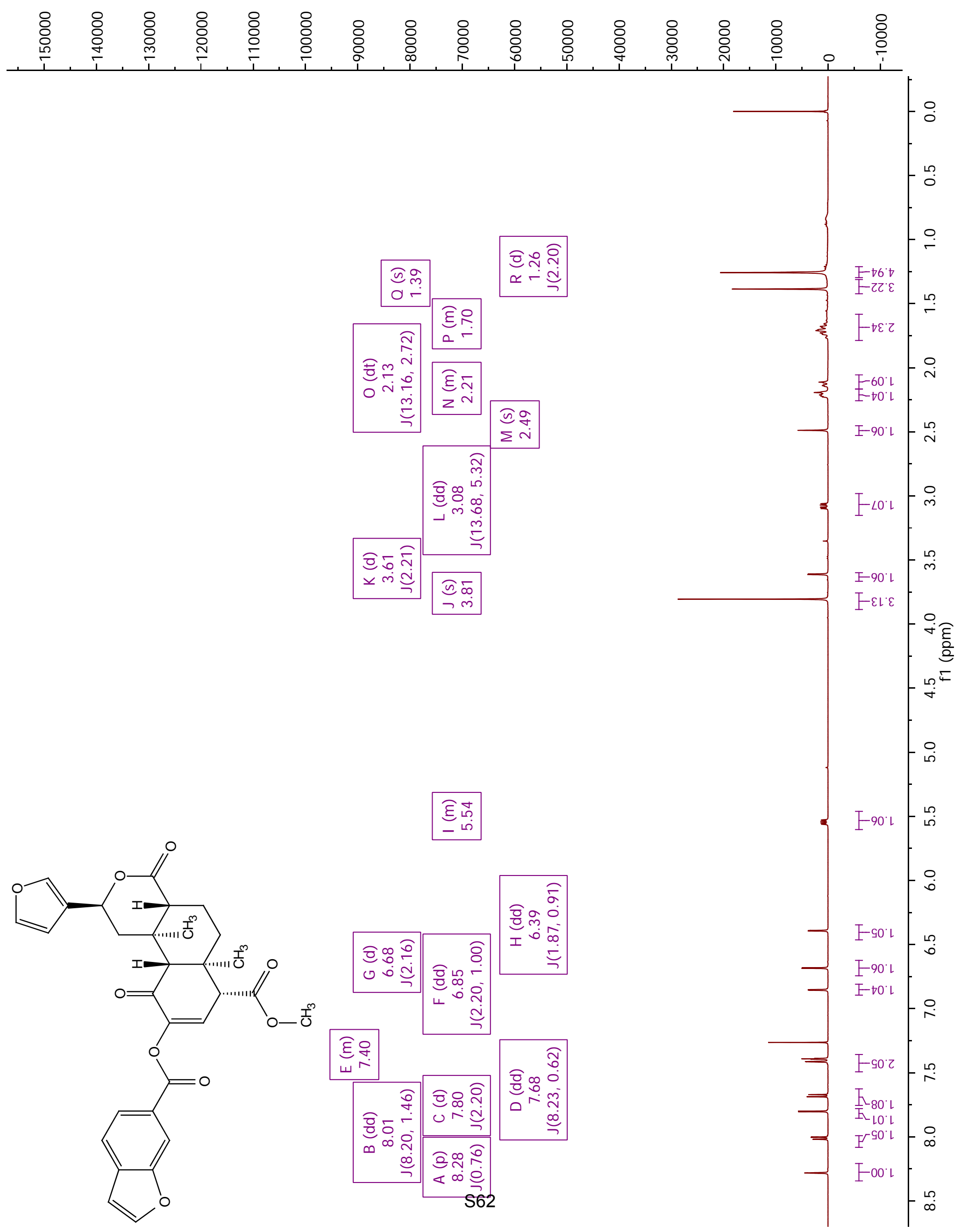




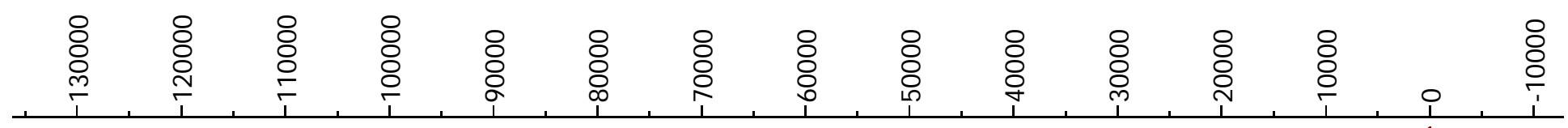

$98^{\circ} \circ \mathrm{L}$
$\nabla 8.9 \mathrm{~L}$

†8'9 -

५८ ऽ६-

$9 t^{\circ} 8 \varepsilon-$

$08^{\circ} \varepsilon t$
$0 C^{\prime} \triangleright t$

8 ' $^{\prime}$ IS-

IS.9S-

9๖'ع9-

$\angle 0^{\circ} Z L-$
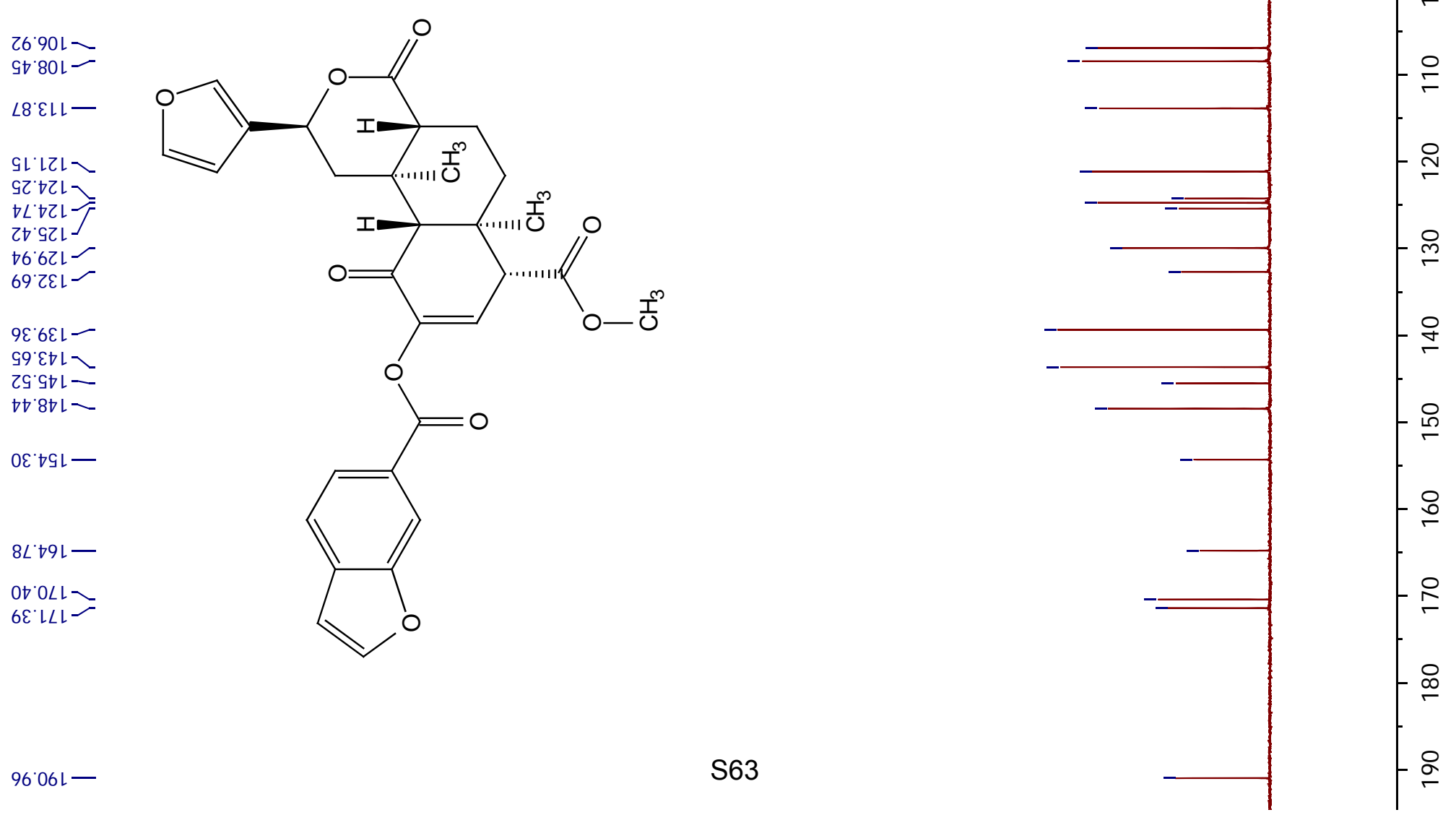


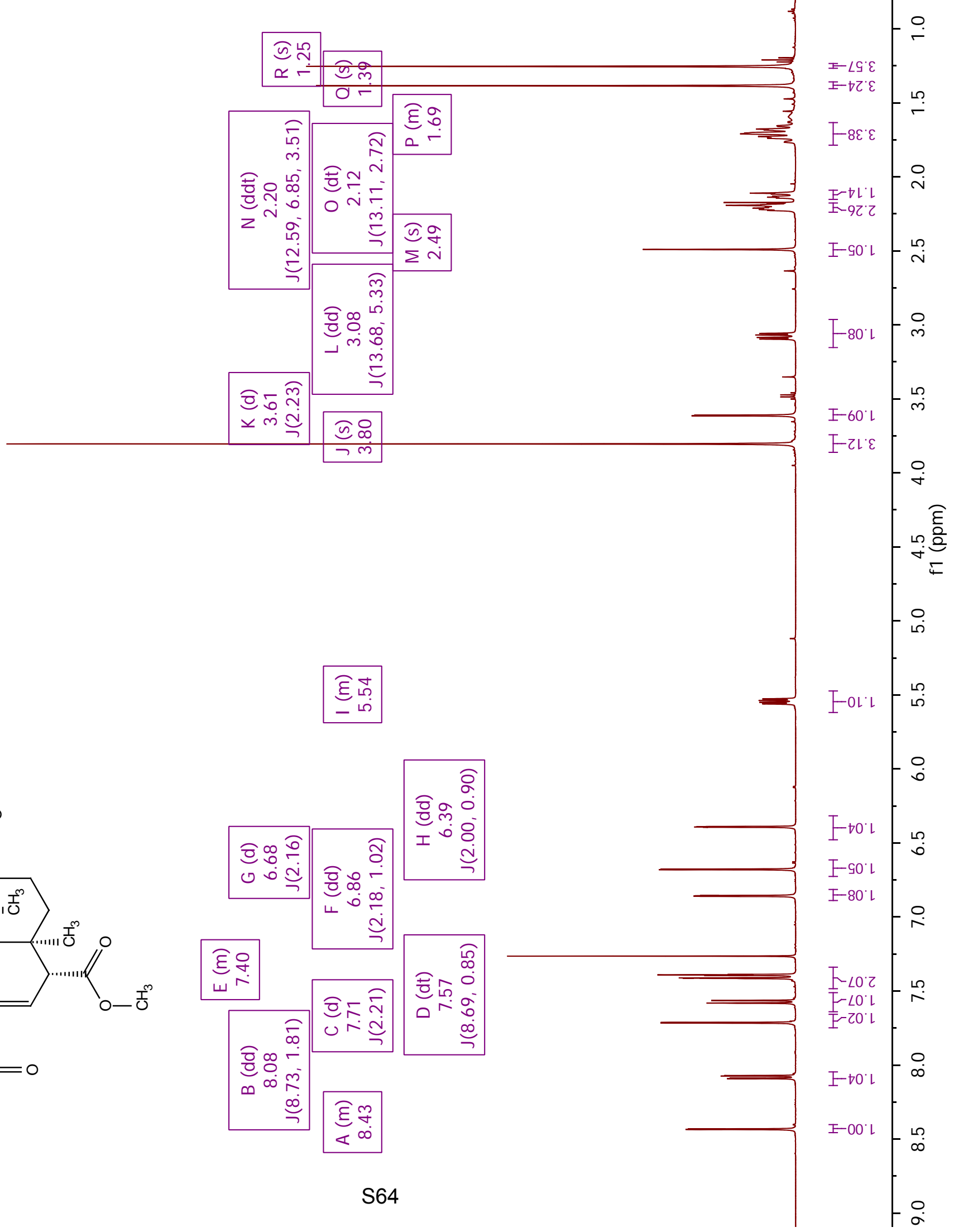




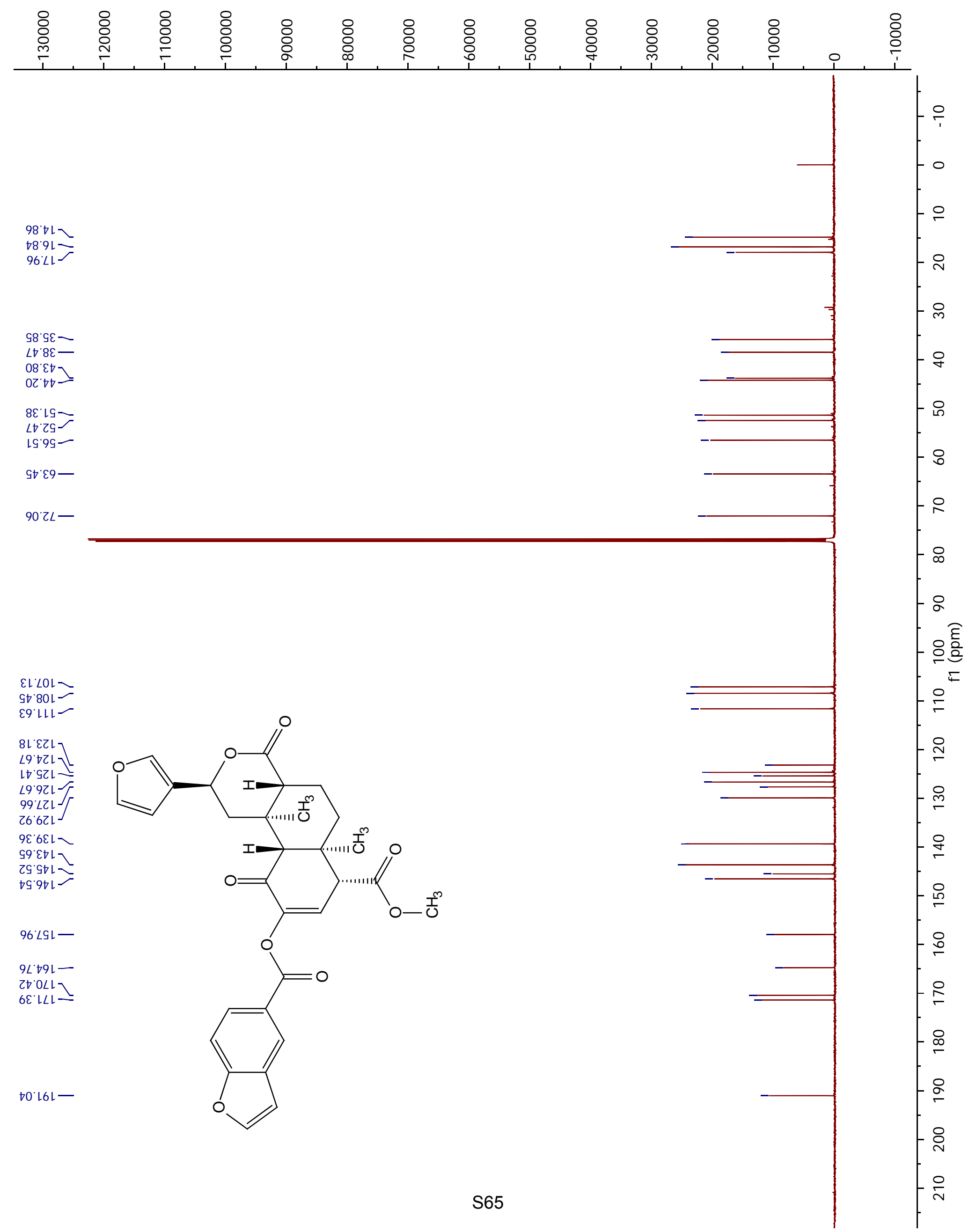




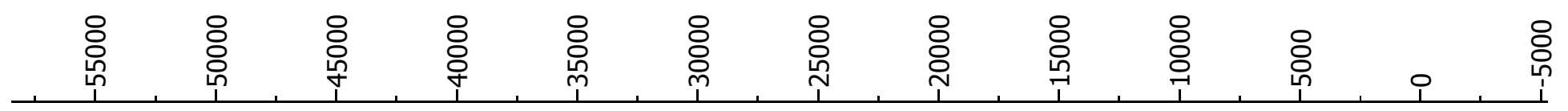
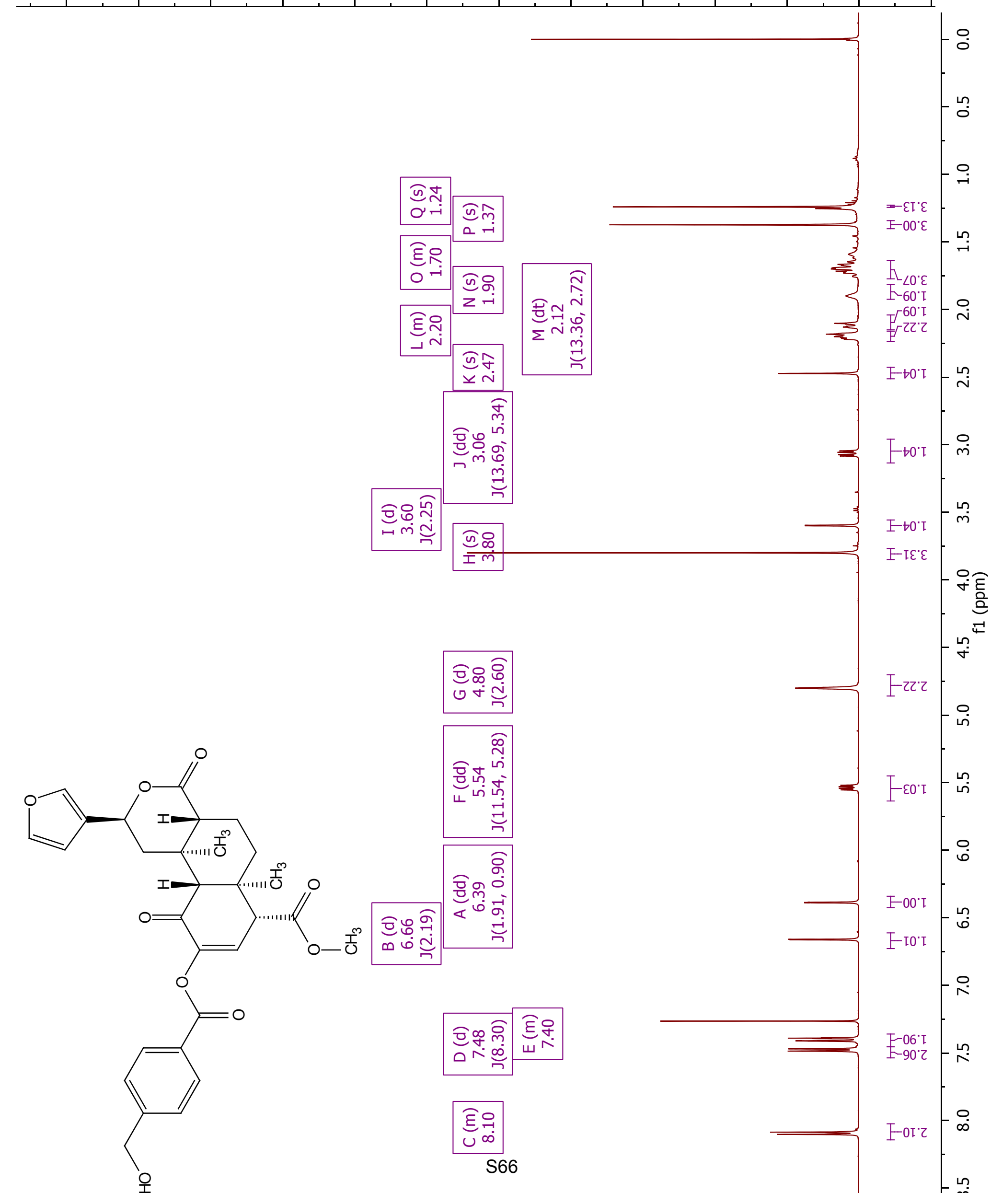


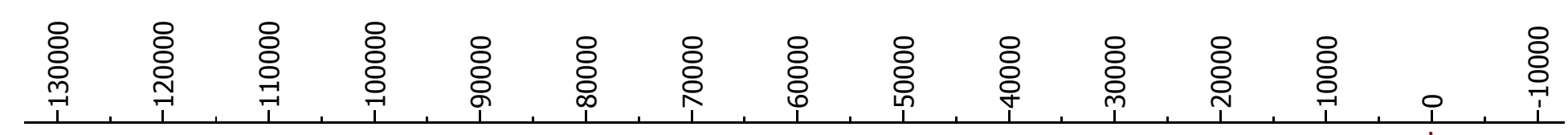

$58^{\circ} \circ \mathrm{I}$
$28^{\circ} 9 \mathrm{I}-$
$\forall 6^{\circ} \angle I$

๑ ${ }^{\circ} \varsigma \varepsilon-$

St $8 \varepsilon-$

6L'Eb>

$6 I^{\circ} t t$

9ع'IS-

$8 b^{2} \mathrm{Cs}$

$6 b^{\circ} 95$ -

เ৮'ع9-

$\angle S+b 9$

$90^{\circ} Z \angle-$

$t t^{\circ} 80 \mathrm{I}$

0t'SZI

SS'9ZI
$\angle E^{\circ} \angle Z I$

66.6ZI 5

$\angle \varepsilon^{\prime} 6 \varepsilon I_{-}$ 99'हt \

Zt.StI-

$\rightarrow I^{\circ} \angle t I-$

0เซ

$6 \varepsilon^{\circ} 0 \angle I$
$0 t^{\circ} T \angle I-$
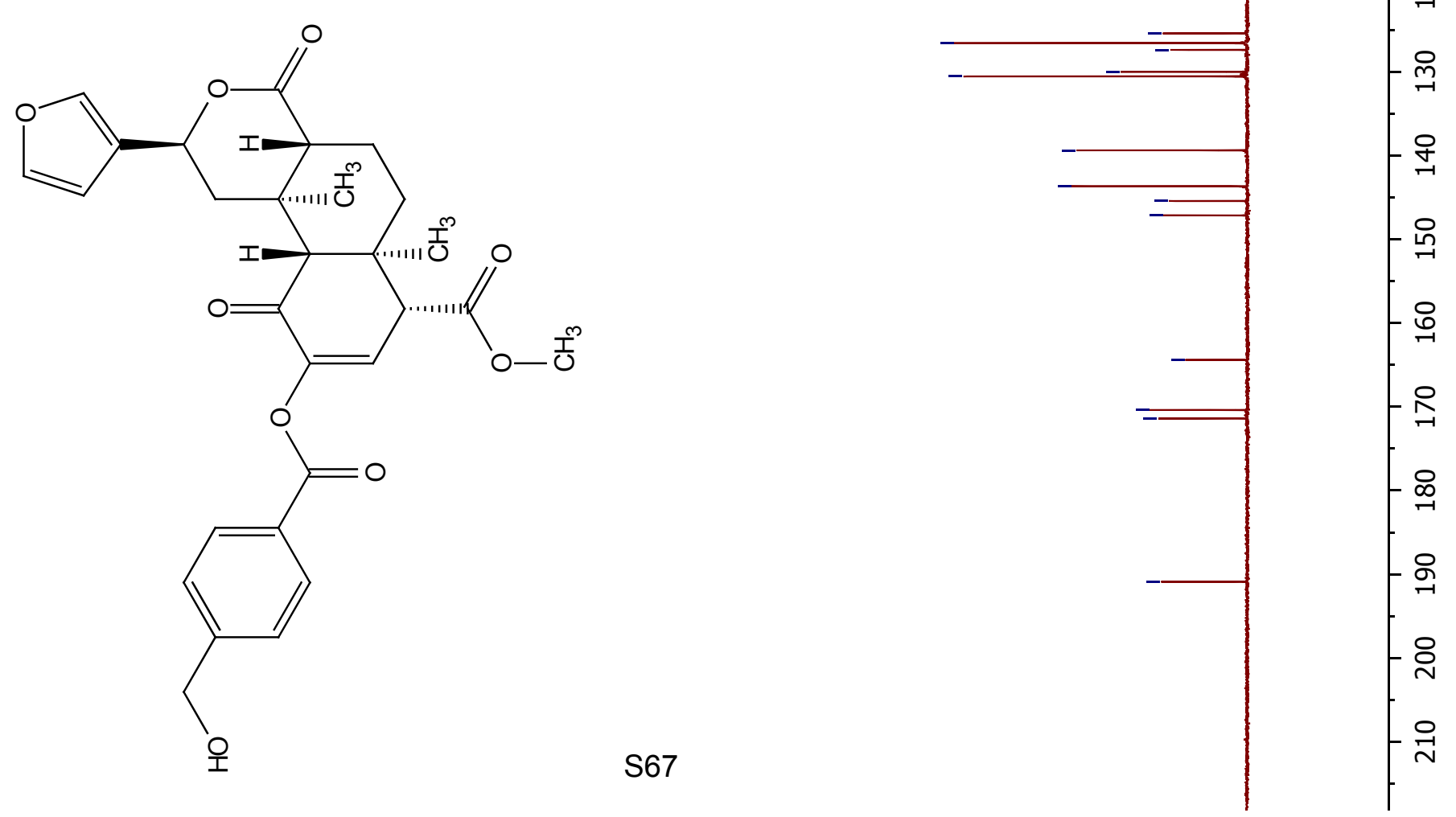


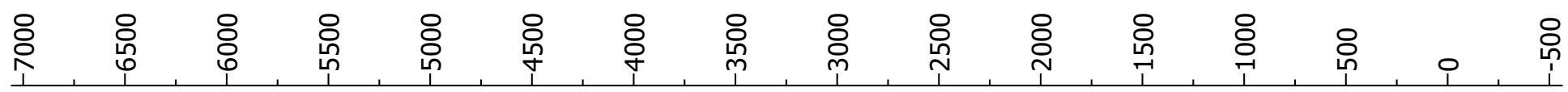
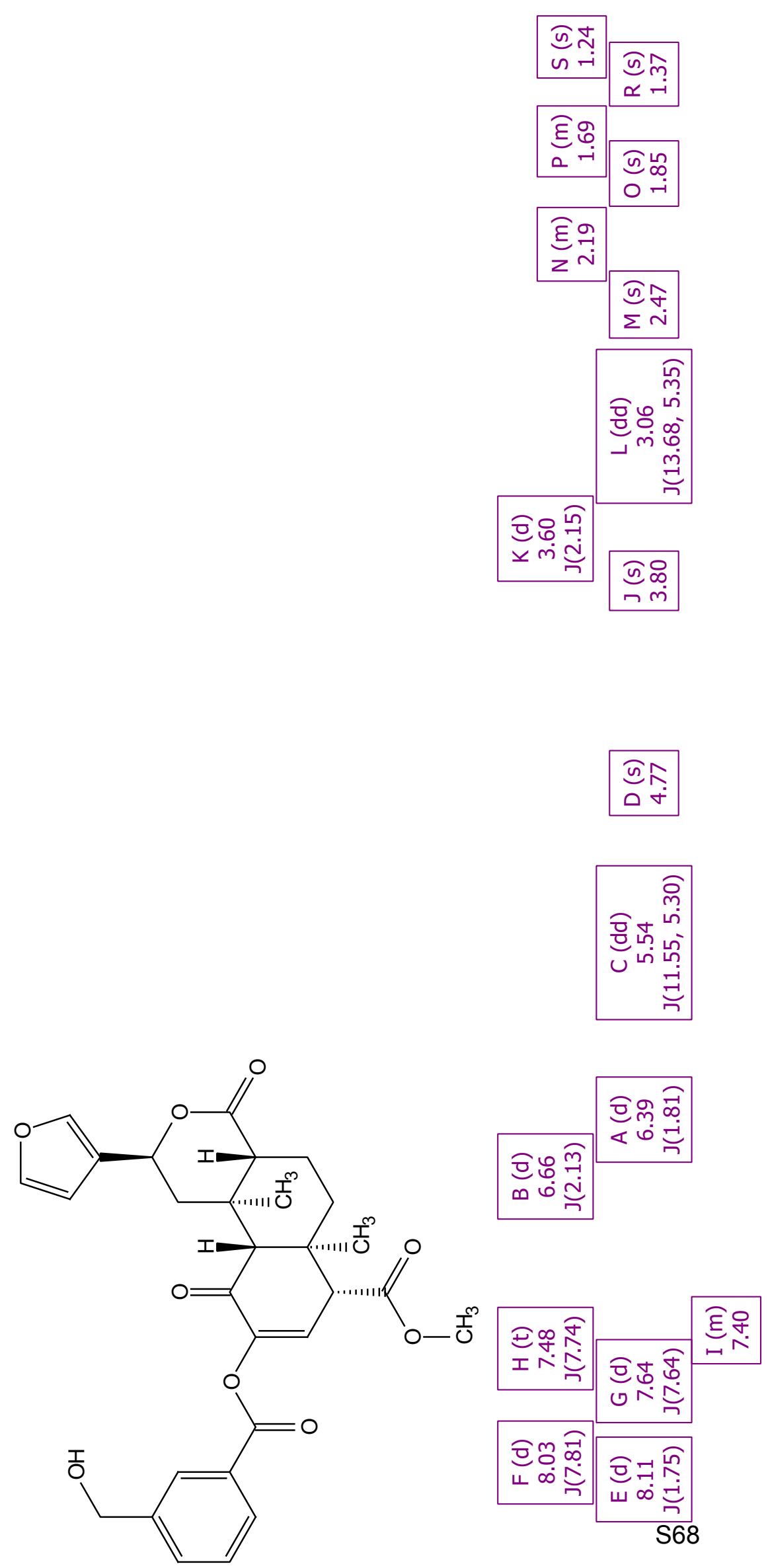

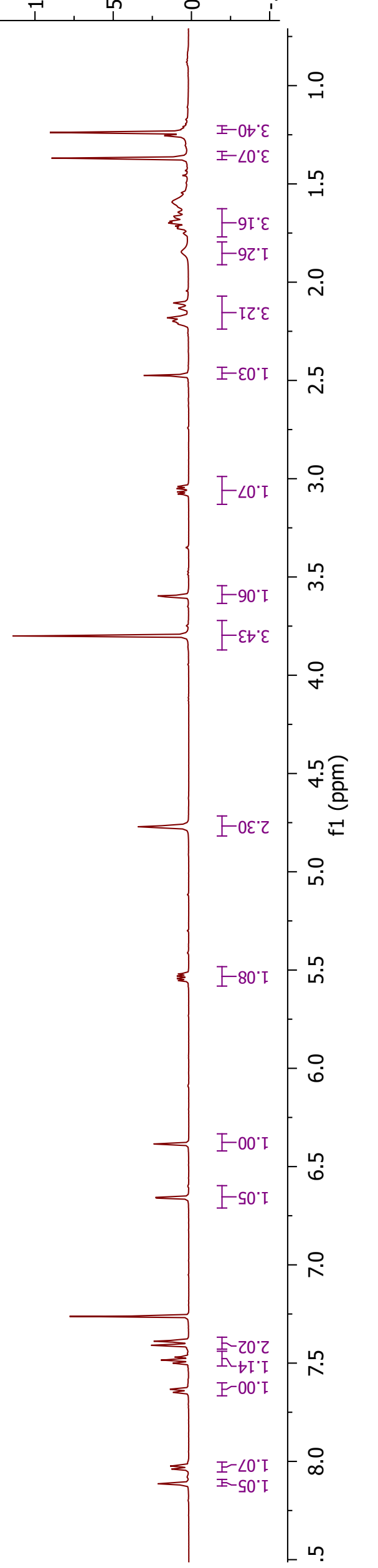




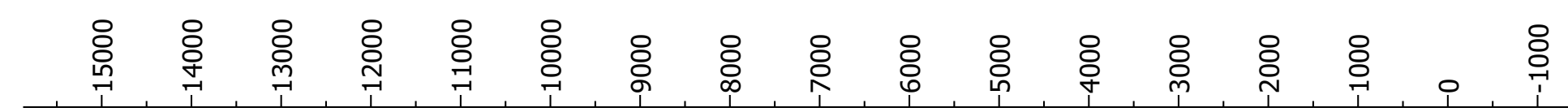

$98^{\circ}+\mathrm{I}$
$28^{\circ} 9 \mathrm{I}$

Z8'9I

$98 . \varsigma \varepsilon-$

$8 t^{\circ} 8 \varepsilon-$

$28^{\circ} \mathrm{Et} \backslash$

$2 C^{\circ} \mathrm{t} \rightarrow$

$8 \varepsilon^{\prime}$ 'IS

$96 \mathrm{TS}$

IS'9S-

6 6. $^{\circ} 9$ -

$\varepsilon 9^{\circ} \downarrow 9-$

$90^{\circ} Z \angle-$
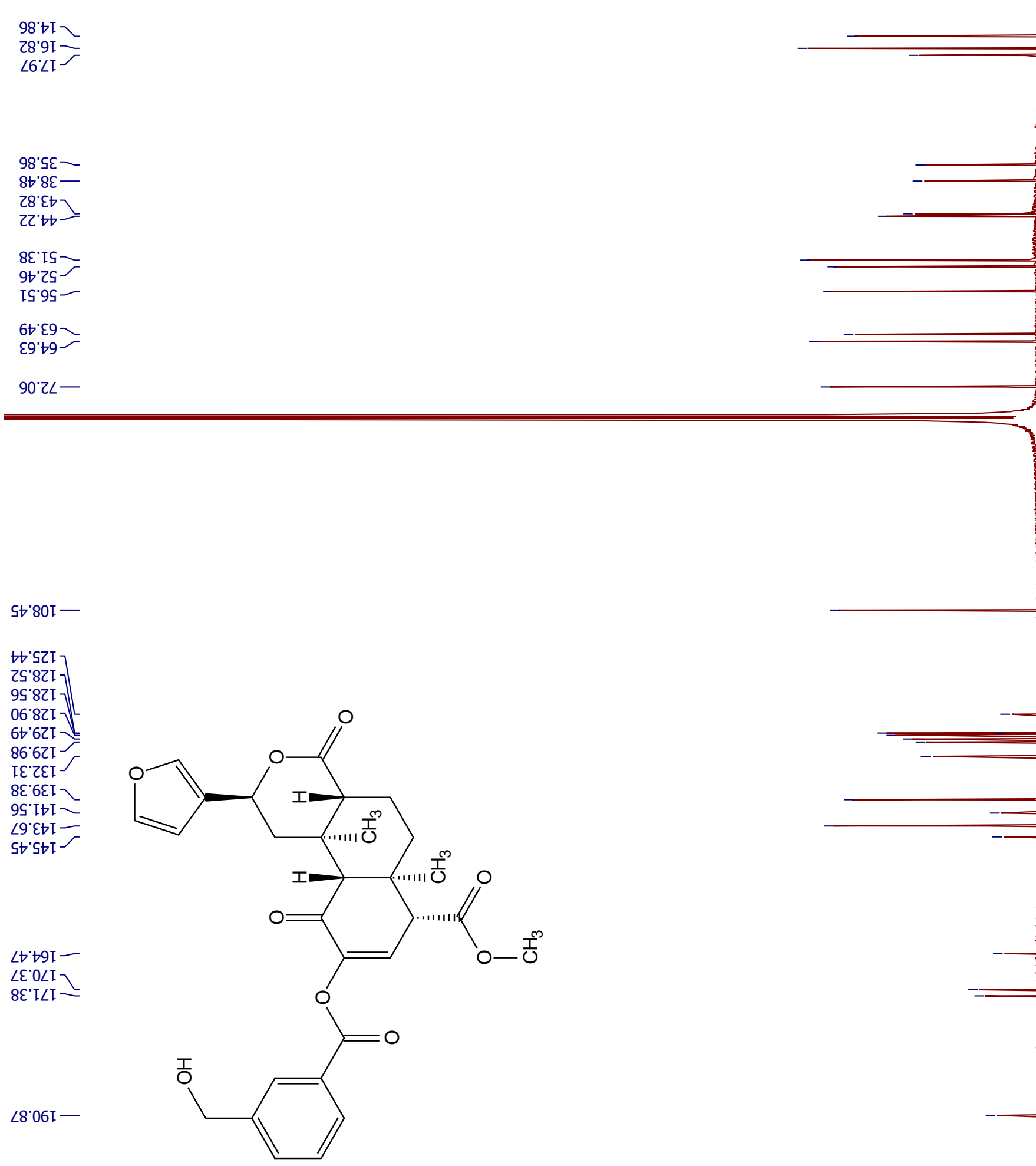


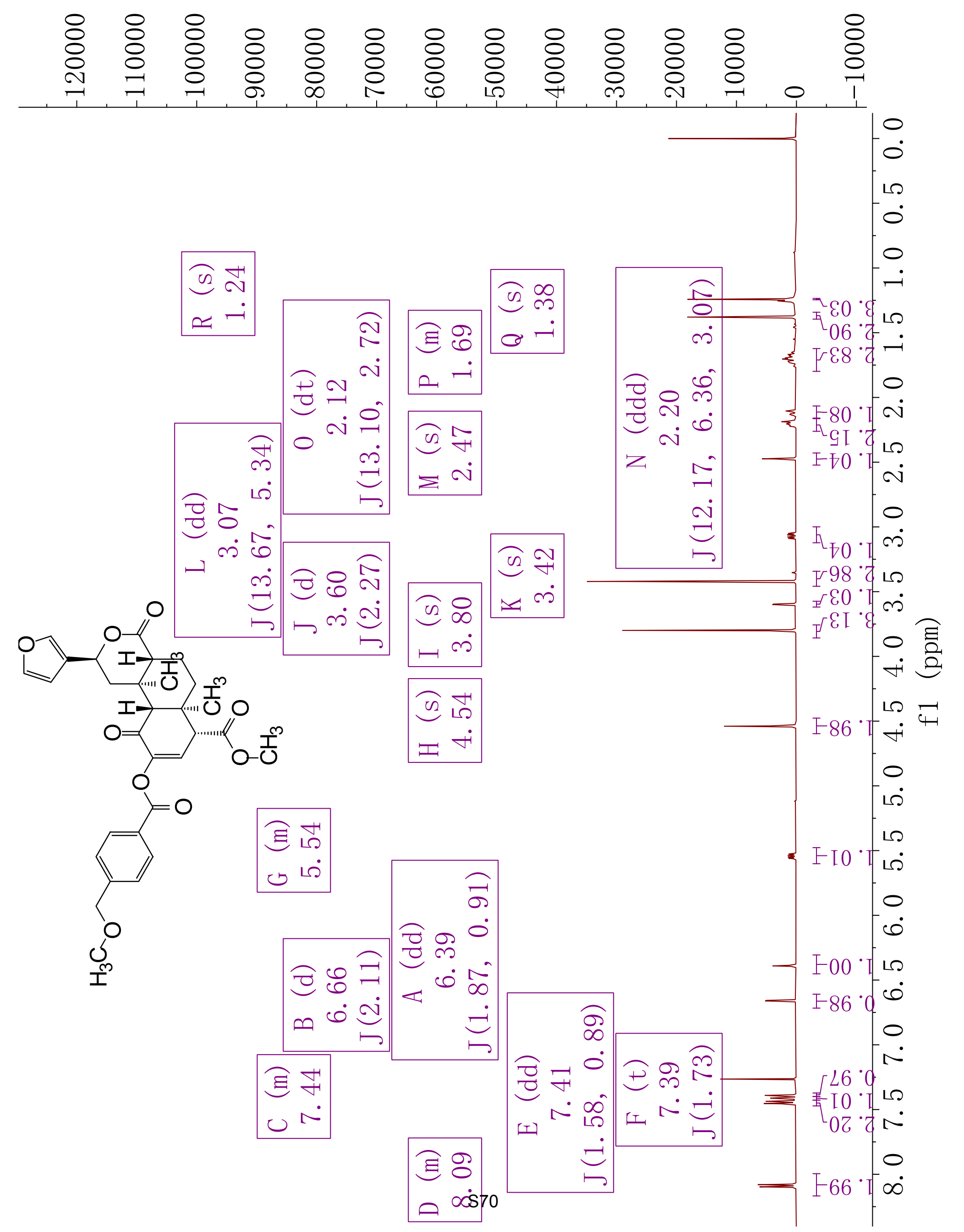




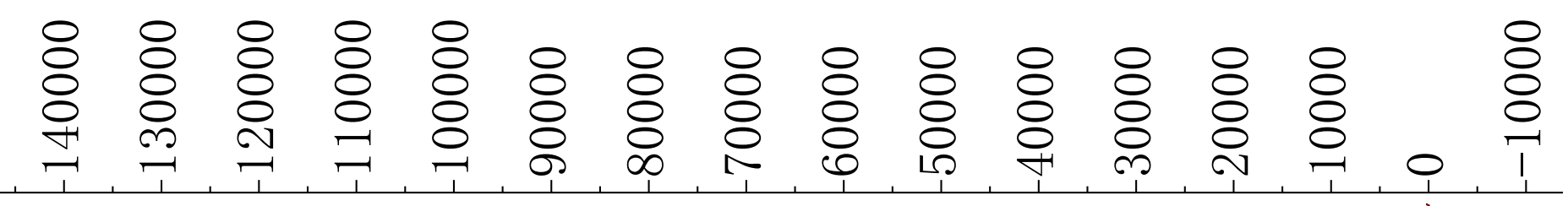

$98^{\circ}$ 五几

$68^{\circ}$. 9 I 5

$78^{\circ} 98$

$97 \cdot 8 \varepsilon \_$

$08^{\circ} \mathrm{ED}$

6 I

LE' I

$\angle T^{\circ} \mathrm{C9^{ \top }}$

67.99

$97 \cdot 89$

97 89

90 ' 245

$06^{\circ} \mathrm{EL}$

切 80 I一

I万 98 I $86^{\circ}$ LZ I ] ZT $\angle Z I \sqrt{2}$ $\angle 6$ 6ZIT Z๐ OE I $9 \varepsilon^{\circ} 6 \varepsilon^{-} \mathrm{I}^{-}$ $99^{\circ} \mathrm{Et}$ 工 朊形工 E下 'Gt [ 矿切 万 $88^{\circ} 0$ L I $8 \varepsilon^{\circ}$ ILI $^{\prime}$

$06^{\circ} 06$ I -
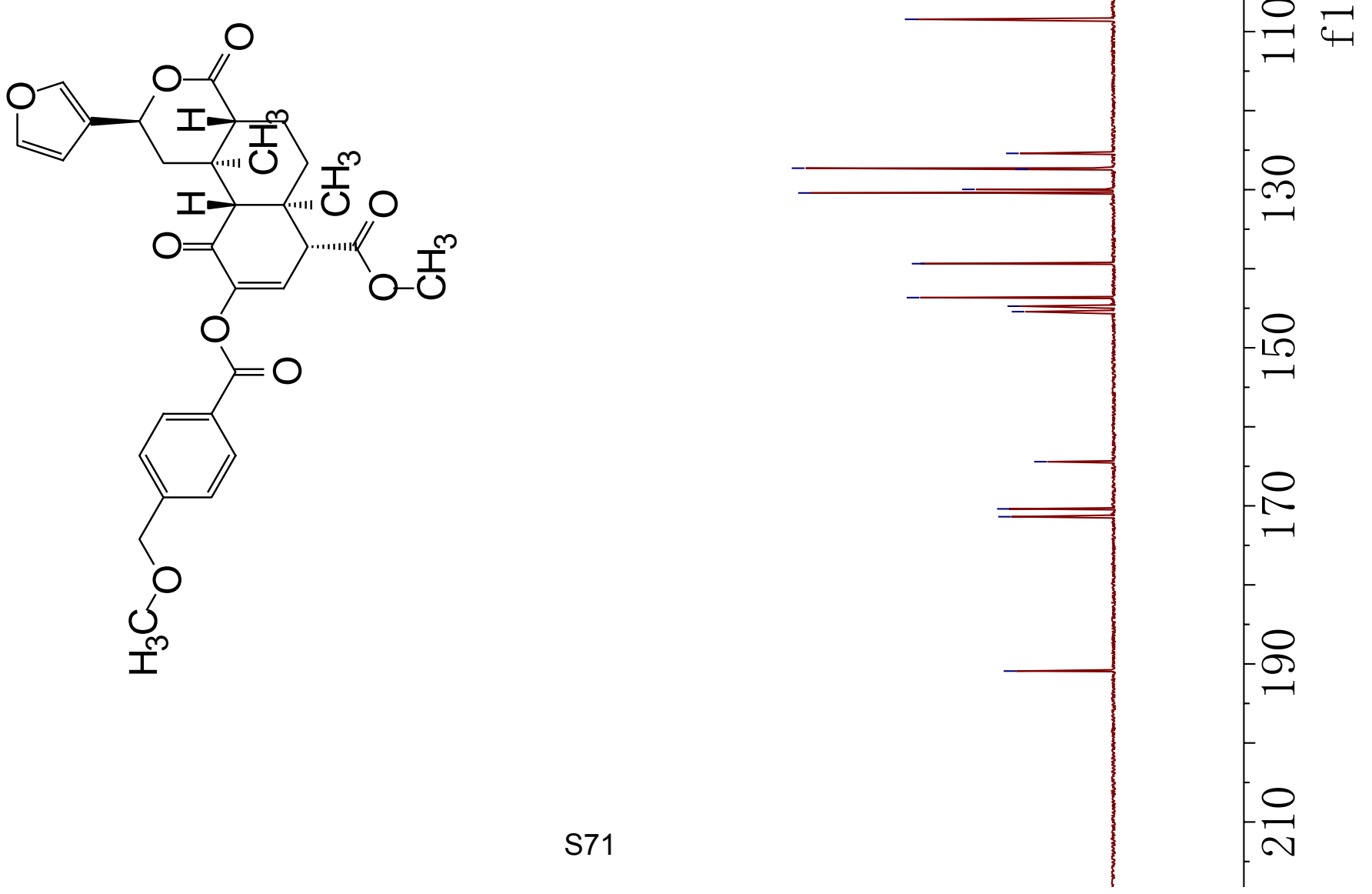

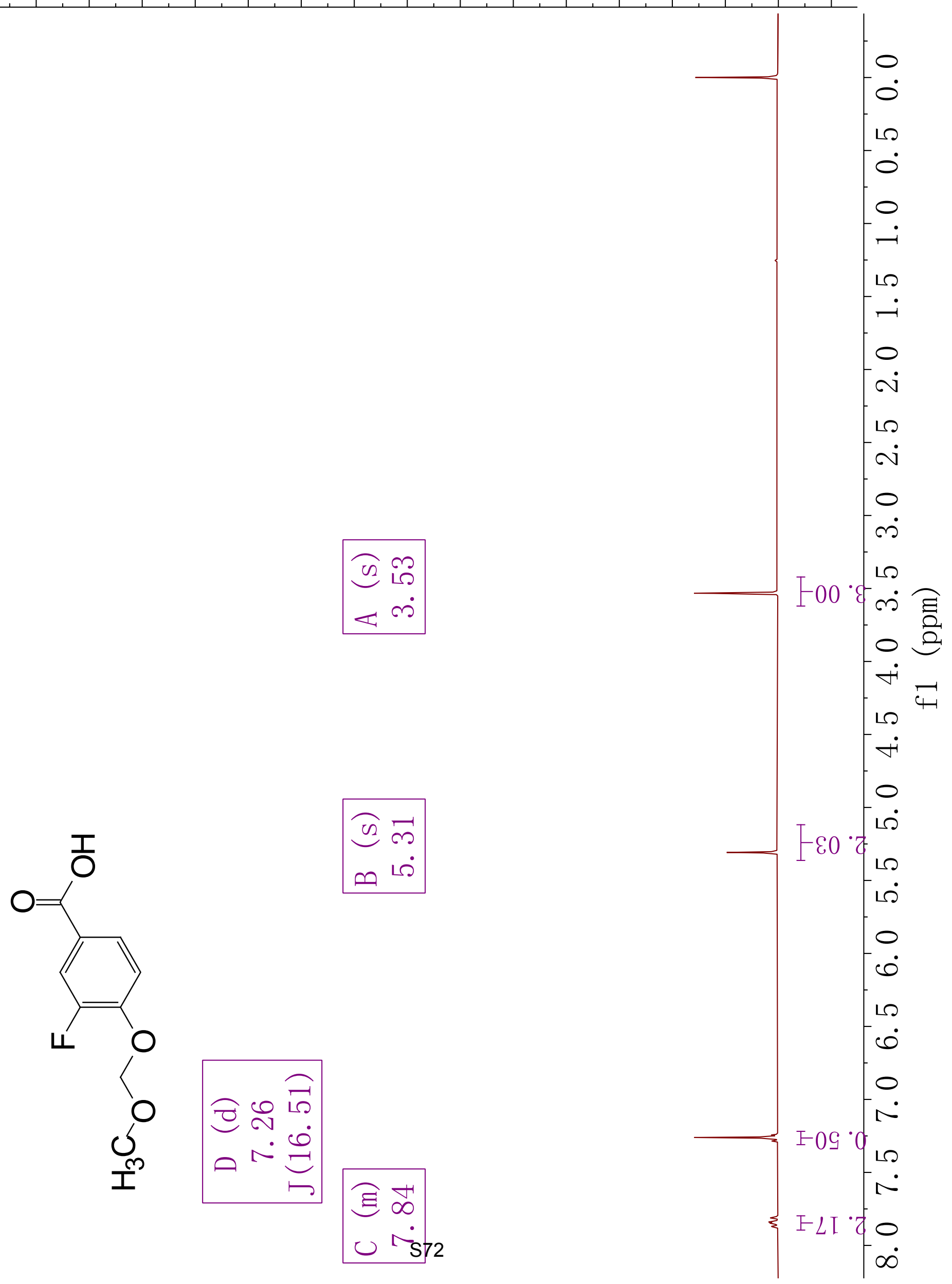


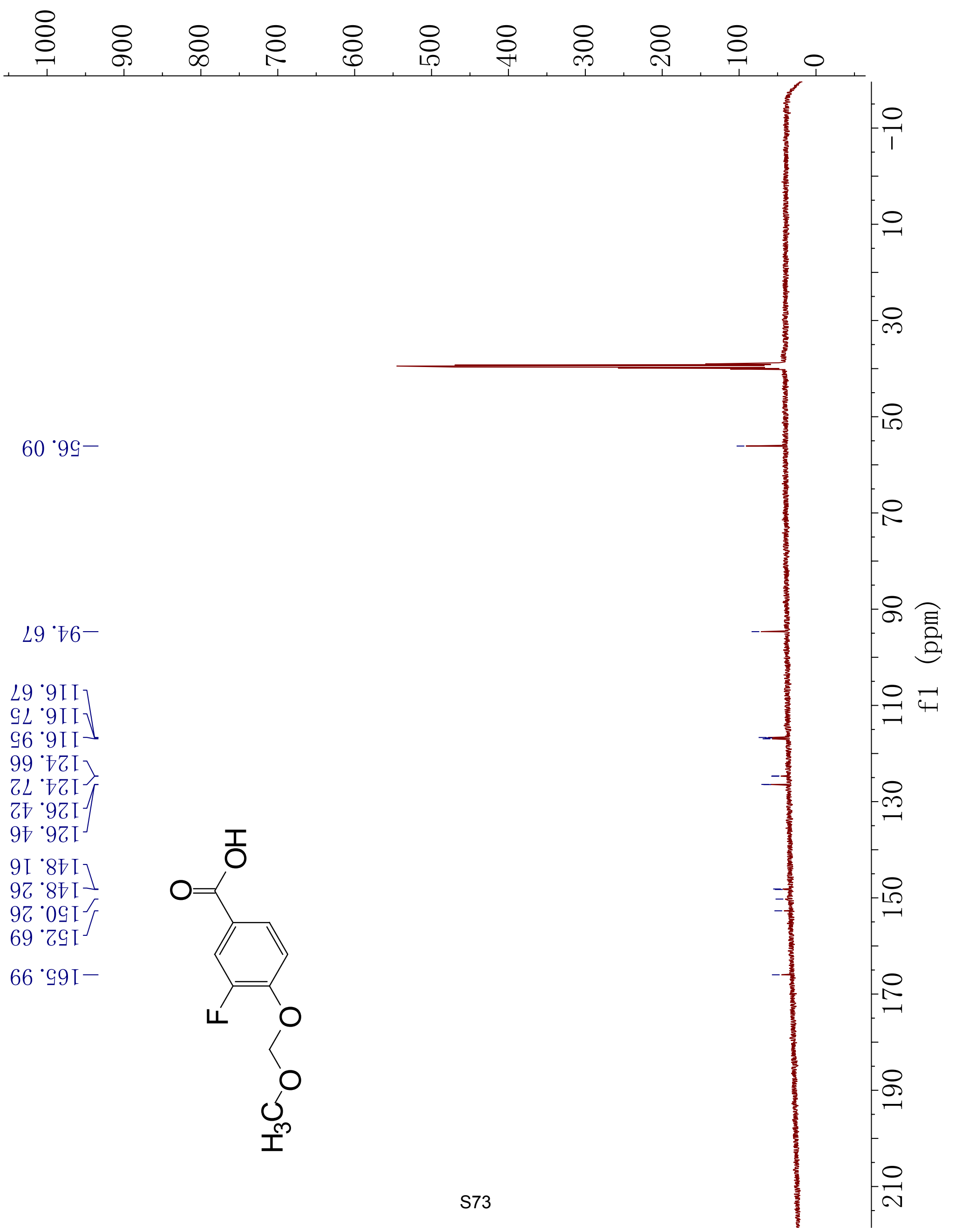




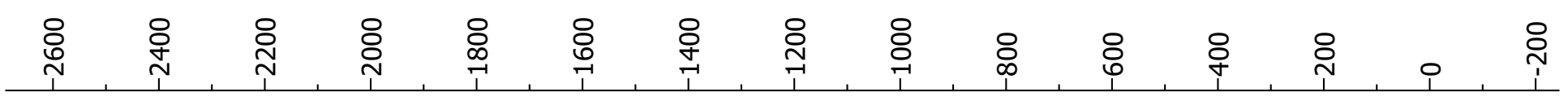

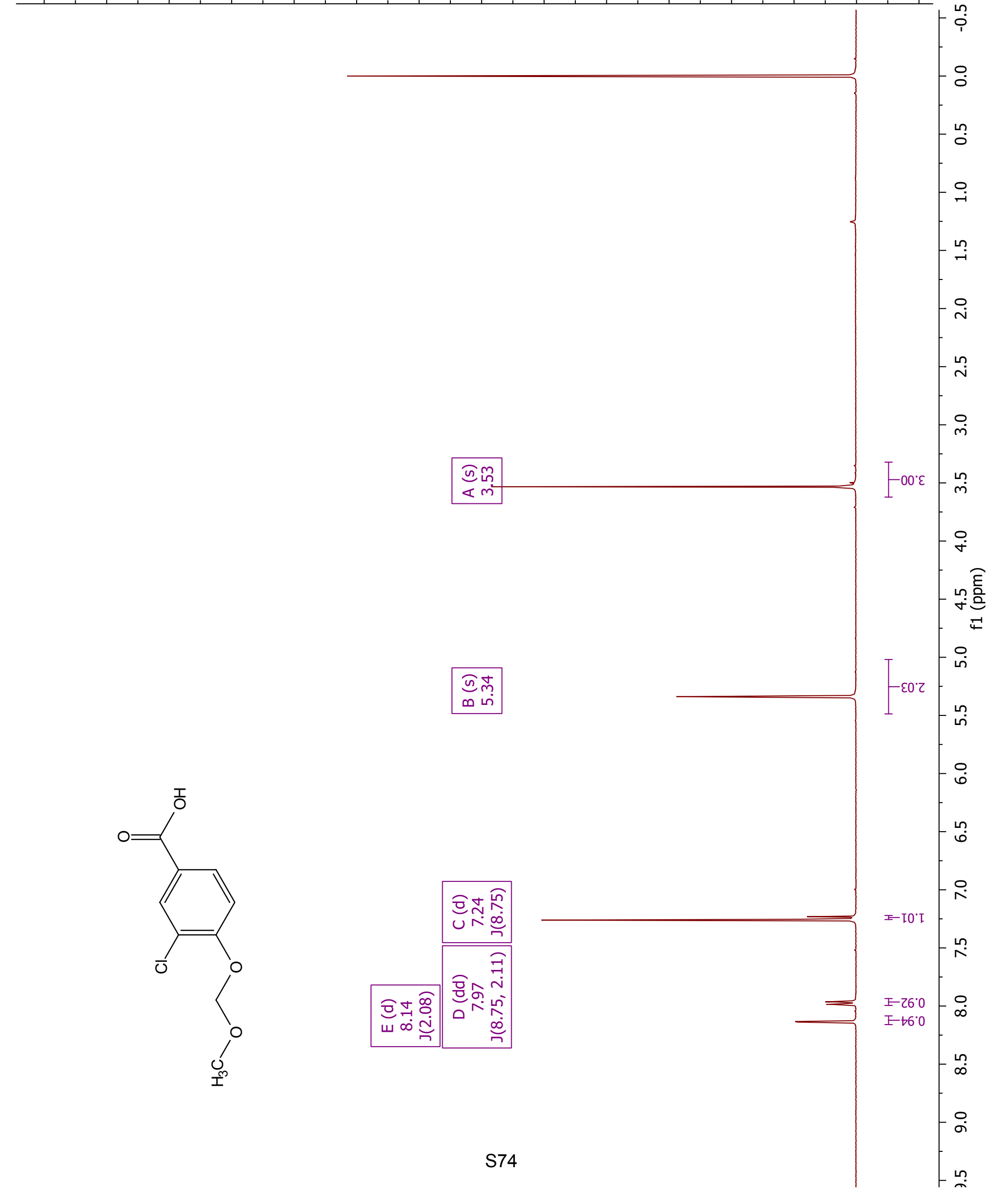




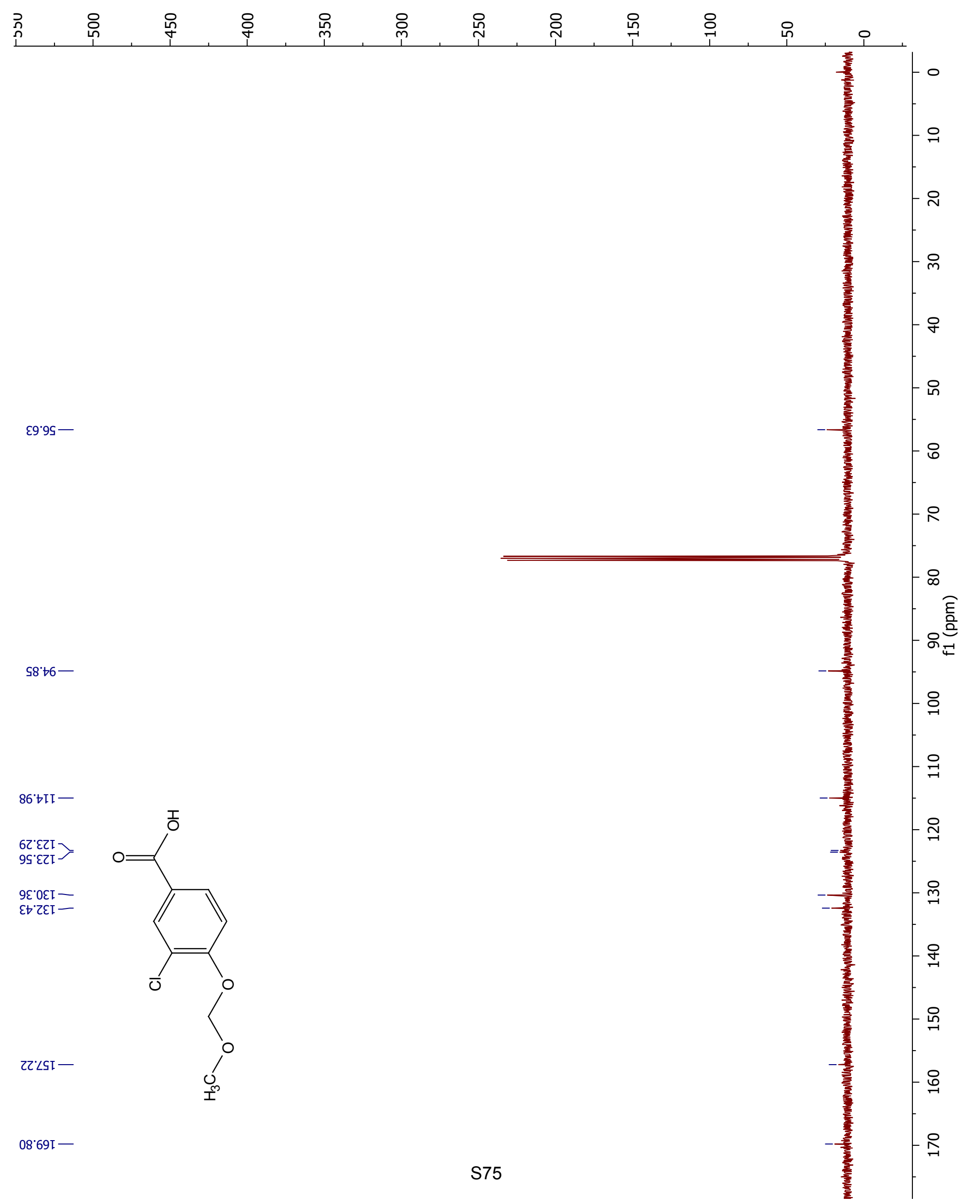




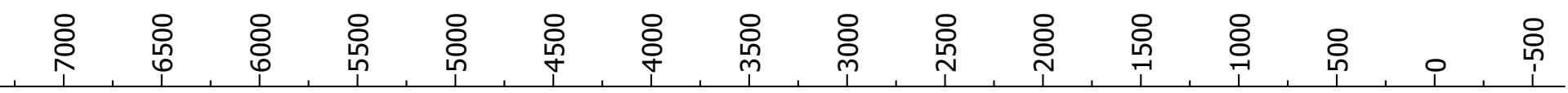
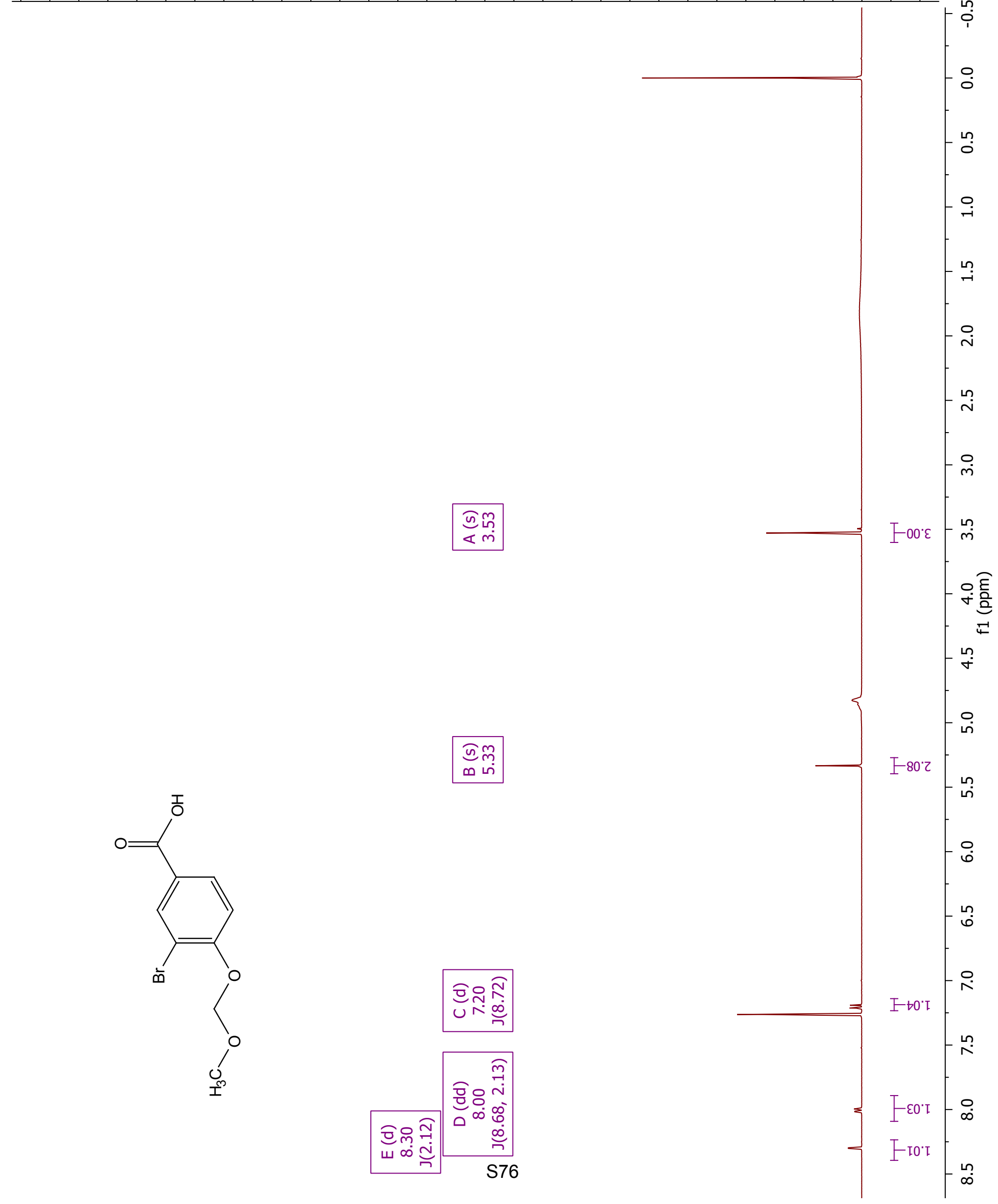


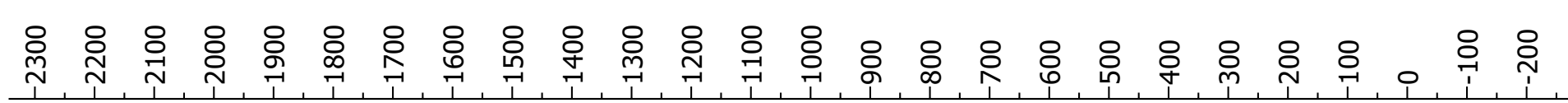

t9. $95-$

$18 \cdot 66-$

IS'ZII-

0L'tII-

0L'EZI -

60.'TET-

8S'SEI -

II'8SI-

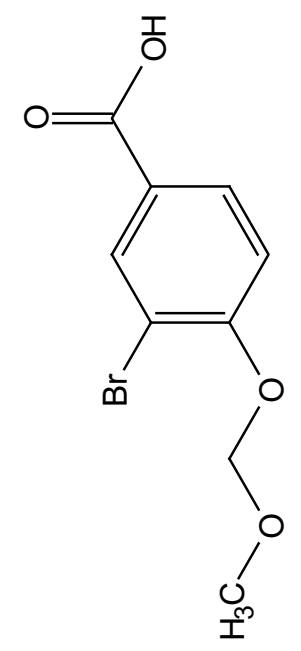

$88^{\circ} 69 \mathrm{I}-$

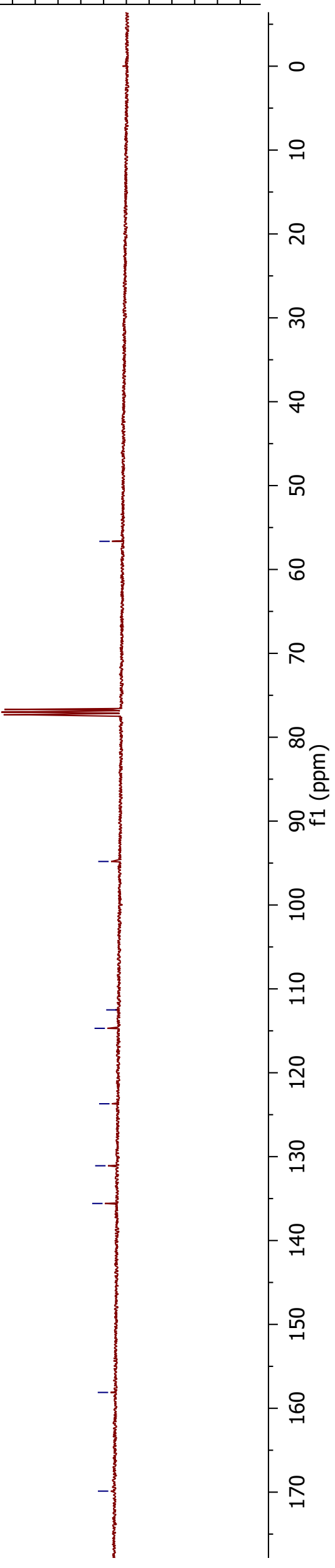




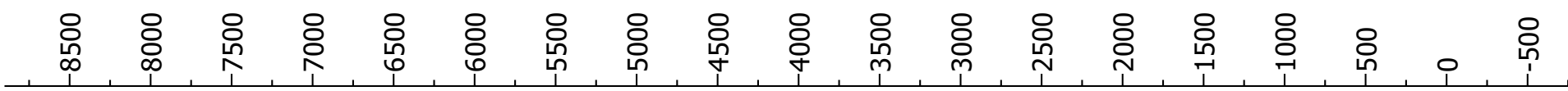

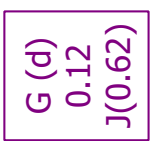

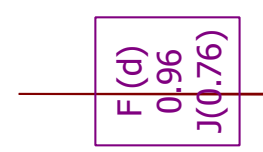

인

ष
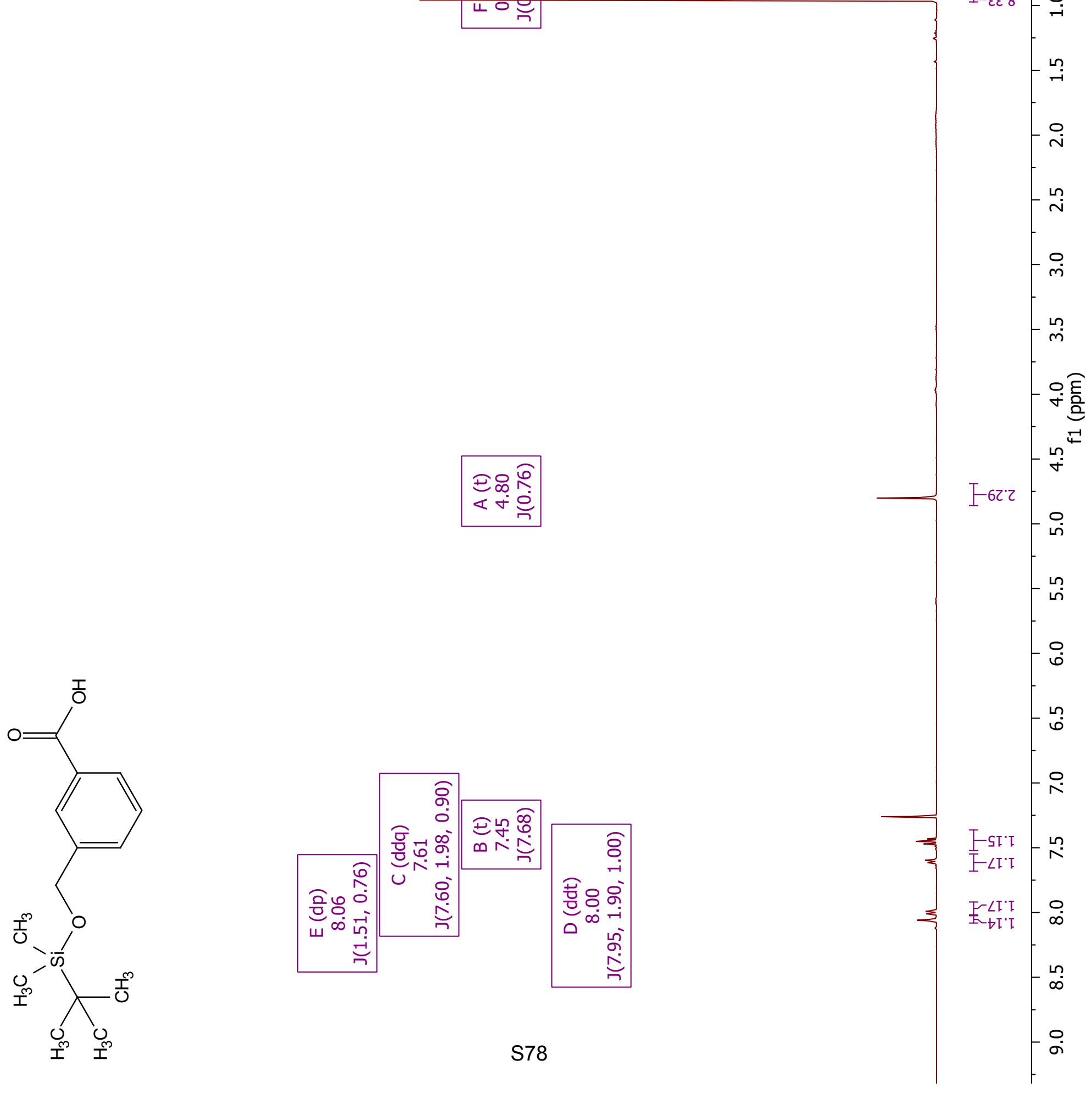


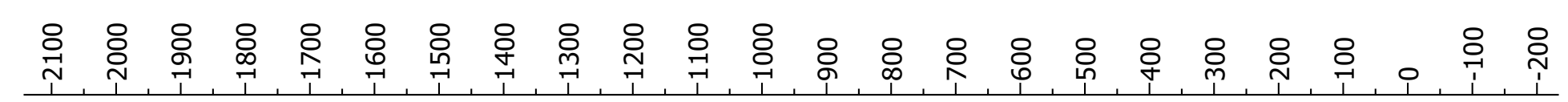

s's- -

$\angle t^{\prime} 8 \mathrm{I}-$

${ }^{\circ} \mathrm{s}-$

$96+59-$

$t \angle \angle Z I]$

$96^{\circ} 82 \mathrm{~T}$

ZZ'6ZI $9 b^{\prime}$.โEI

เ๐'て๖I-

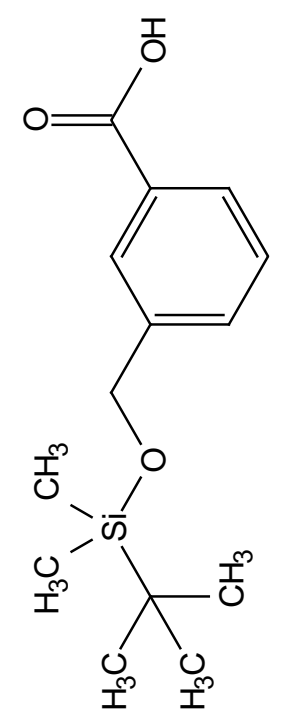

$t 6^{\circ} \mathrm{T} \angle \mathrm{I}-$

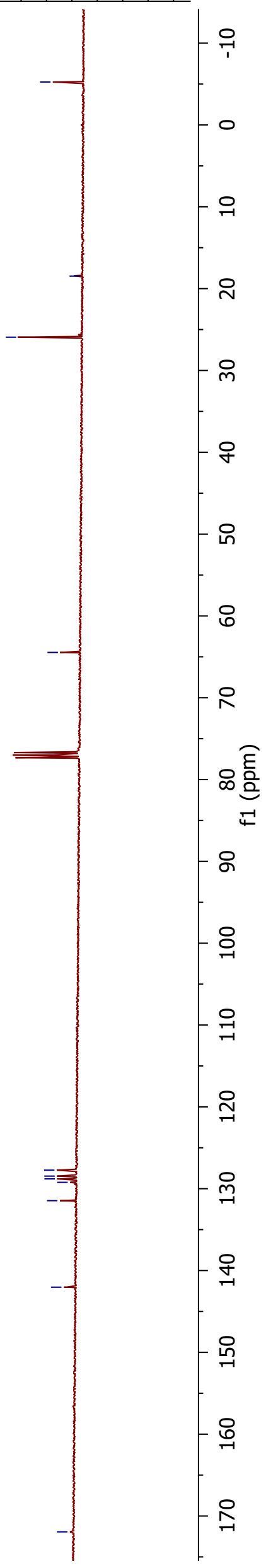


HPLC Traces 

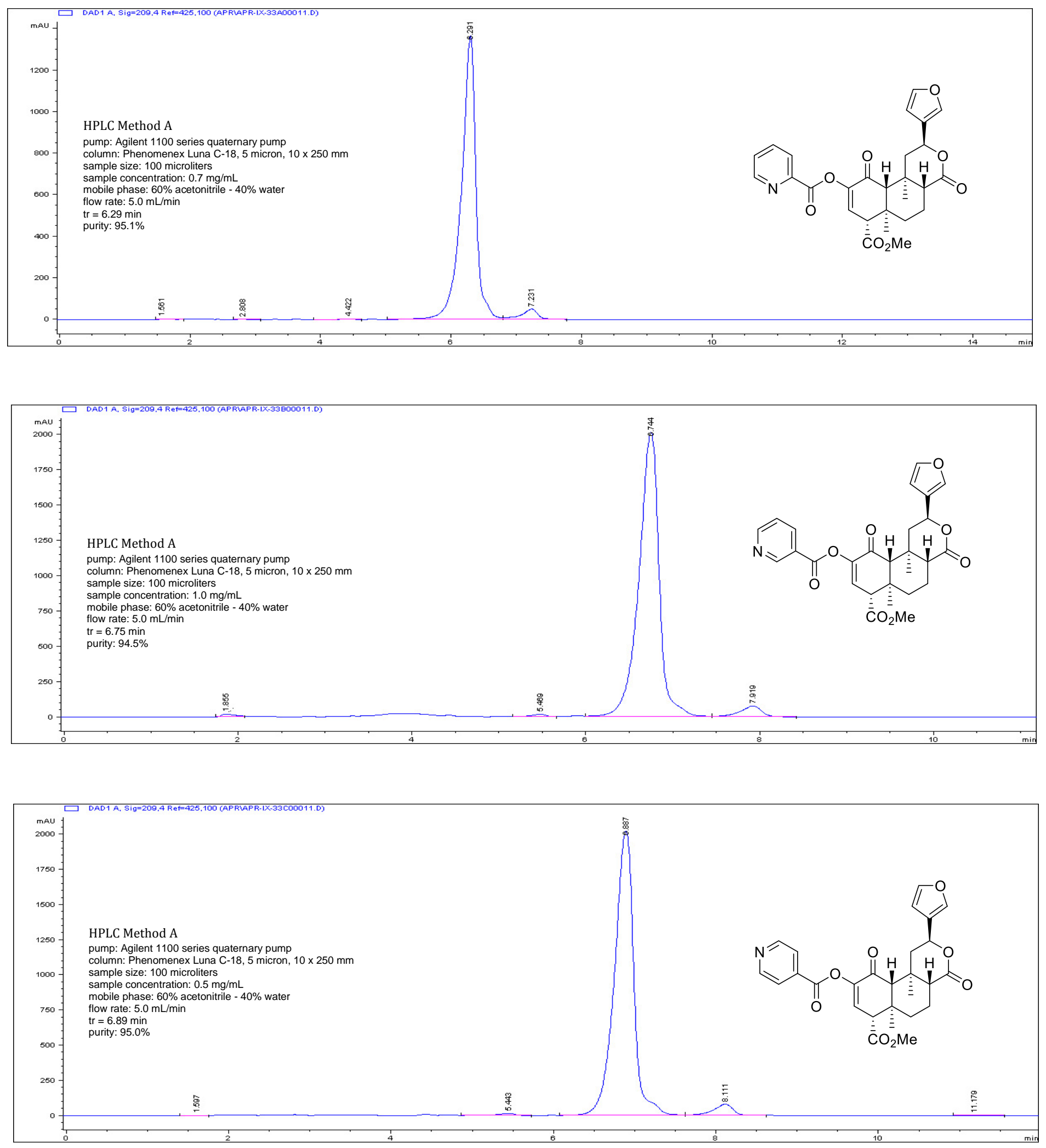

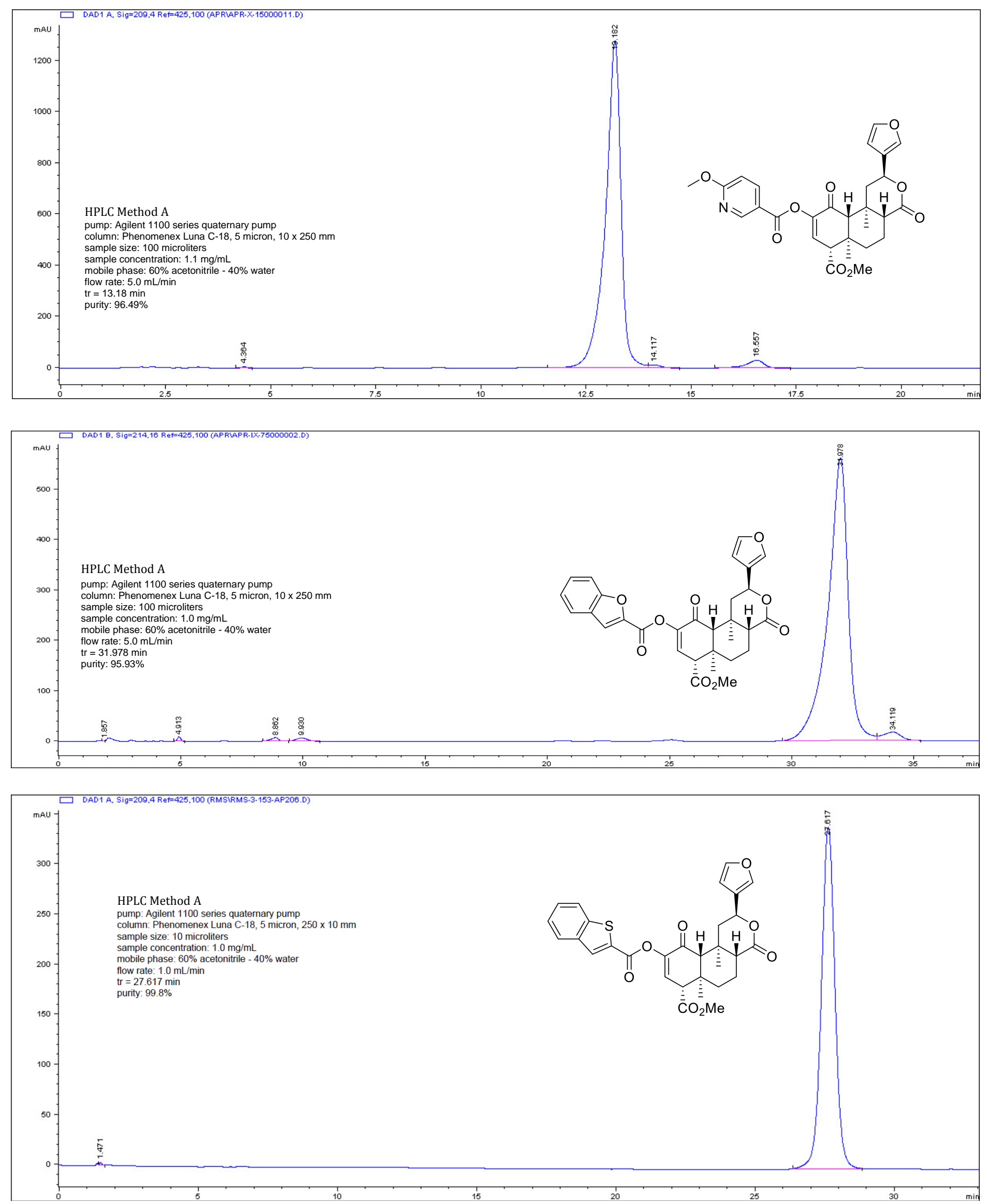

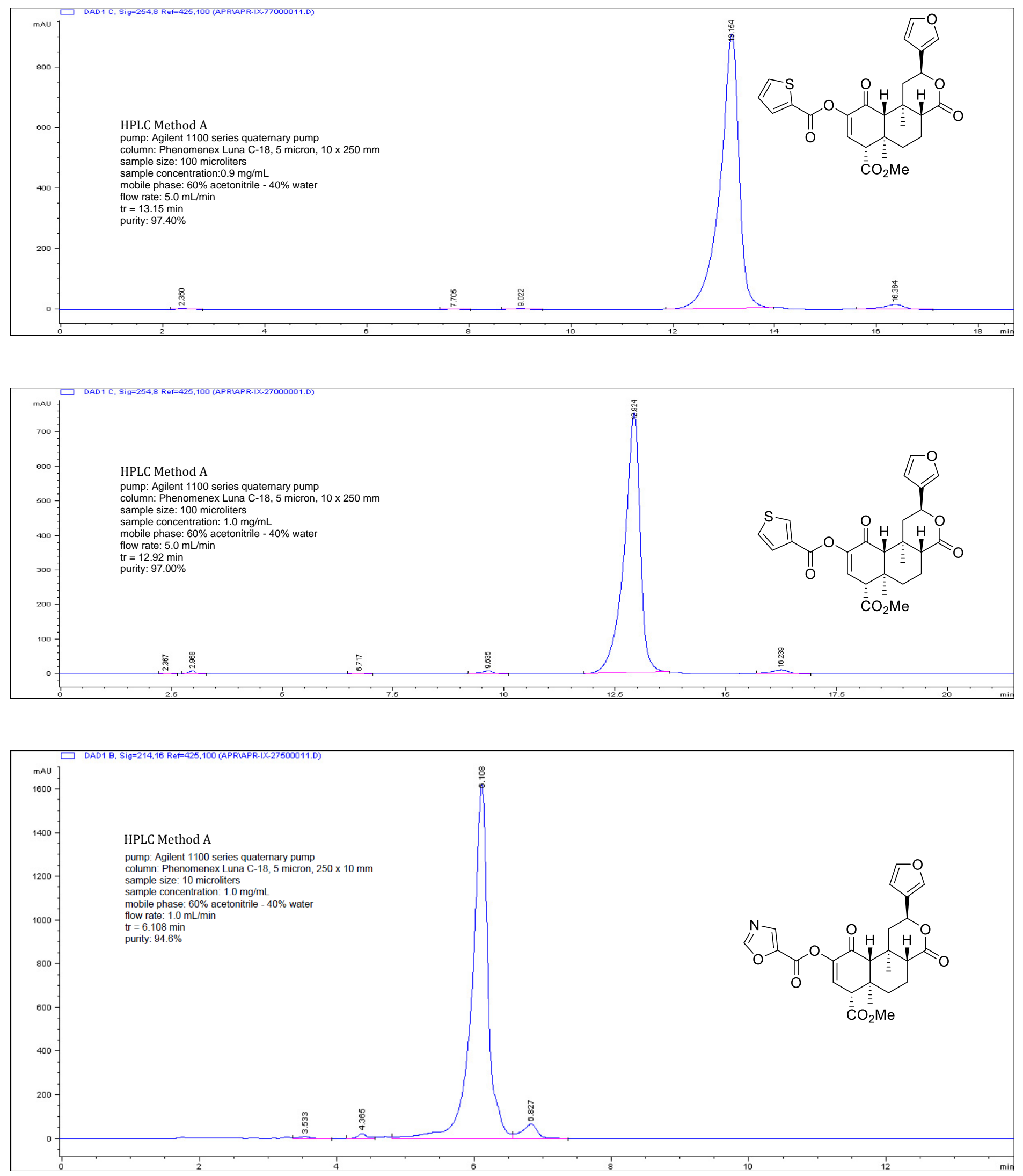

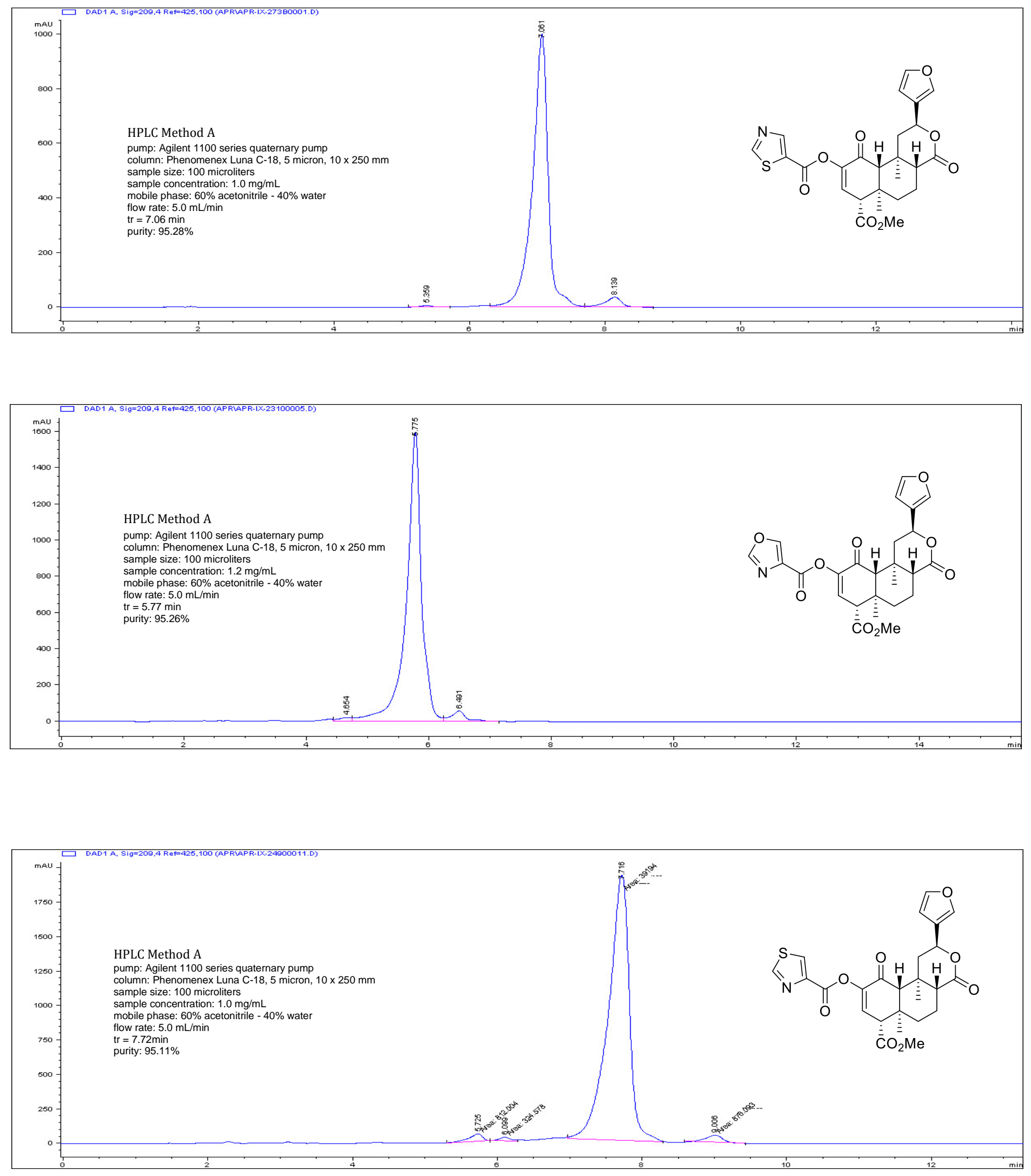

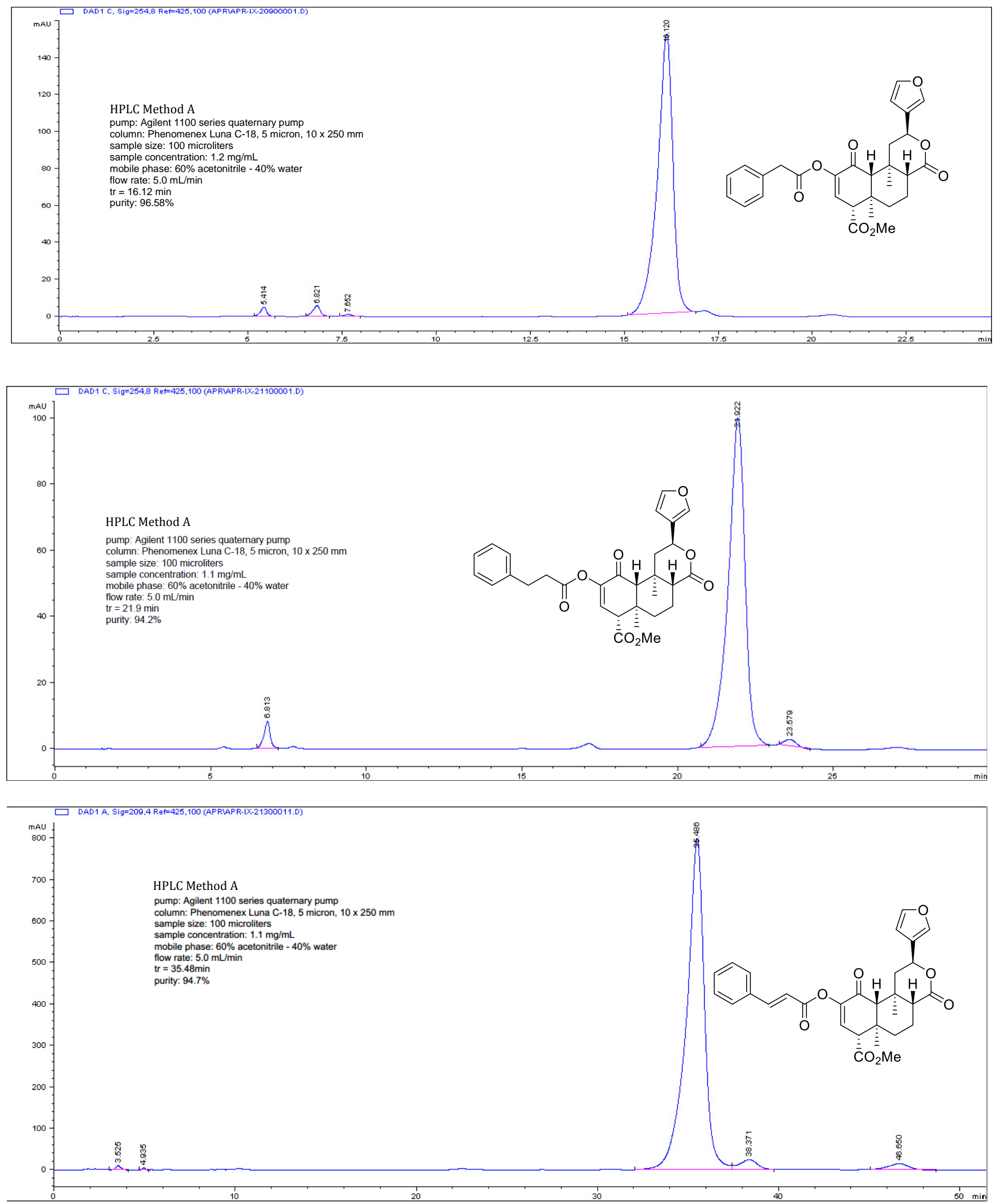


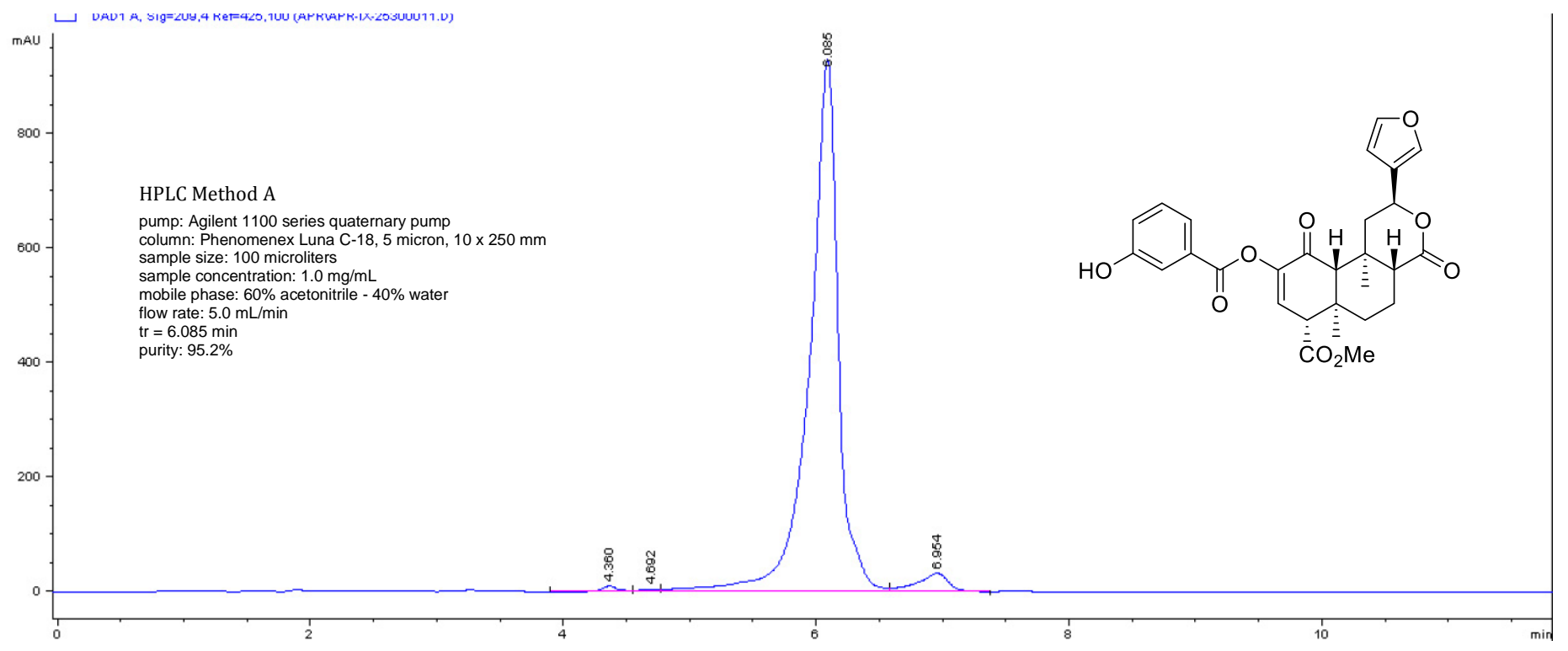

ID DL-1-104-F2AB File DL180105WT007 Date 04-Jan-2019 Time 13:29:57 Description SMDF106833

3: UV Detector: 214
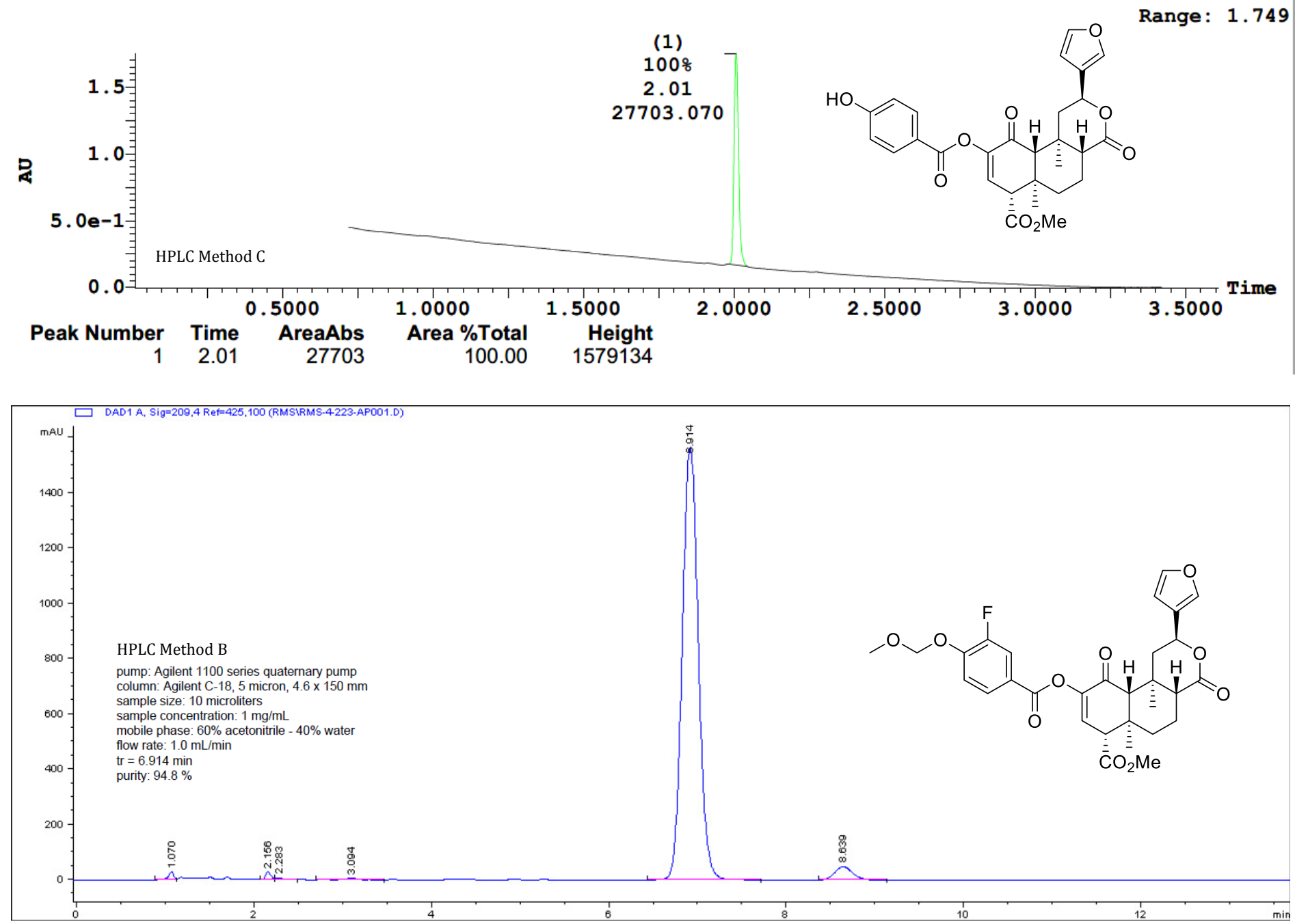


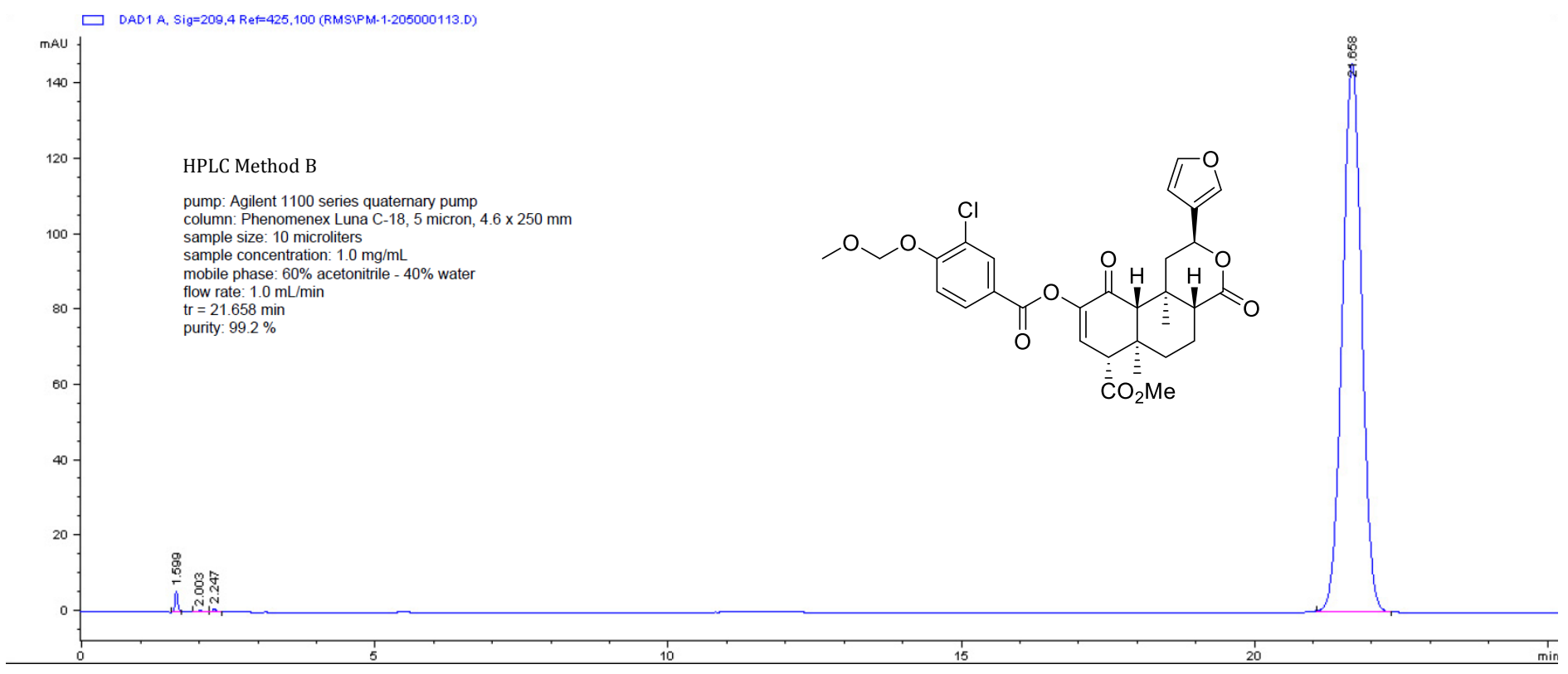

ID PM171_PREP File RC170510WT004 Date 11-May-2017 Time 12:09:22 Description CCT134547

3: UV Detector: 214

$8.515 e-1$

Range: $8.515 e-1$

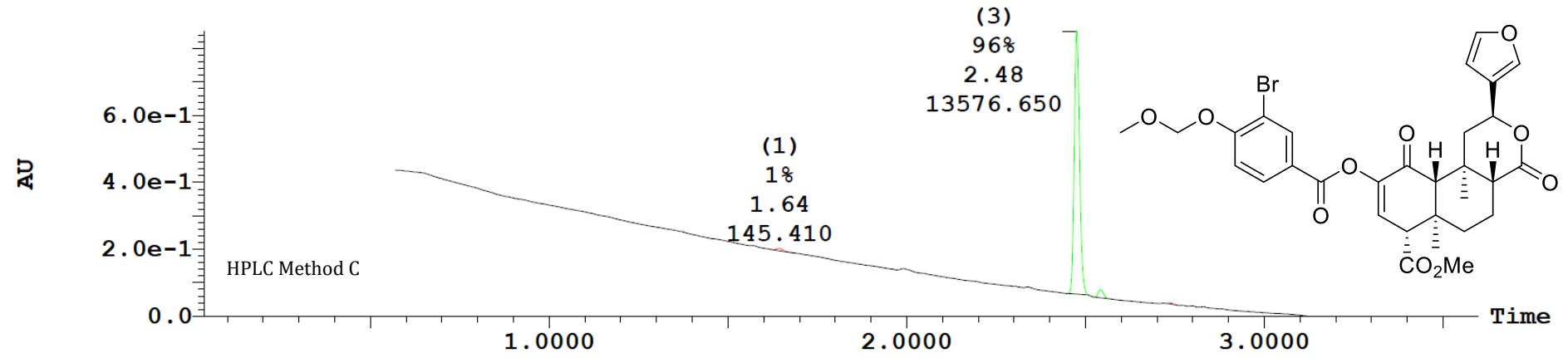

ID RMS-5-55 File RC170309WT013b Date 09-Mar-2017 Time 18:17:38 Description CCT138263

3: UV Detector: 214

1. $604 \mathrm{e}-1$

Range: $1.603 e-1$

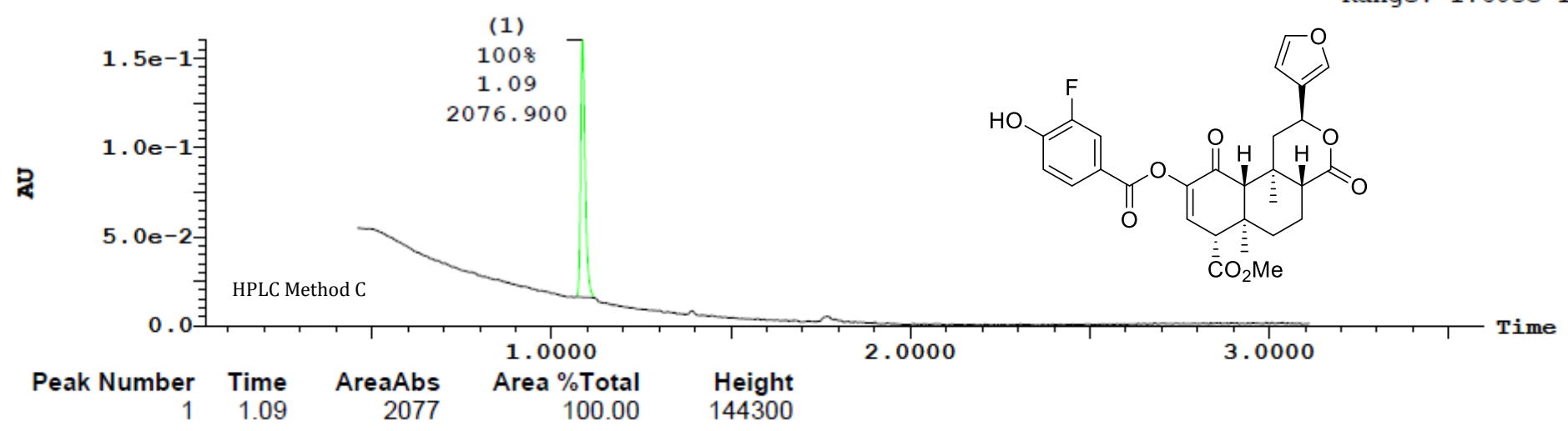


ID RMS-5-117 File RC170421WT012 Date 24-Apr-2017 Time 16:18:44 Description CCT130540

3: UV Detector: 214

$6.062 \mathrm{e}-1$

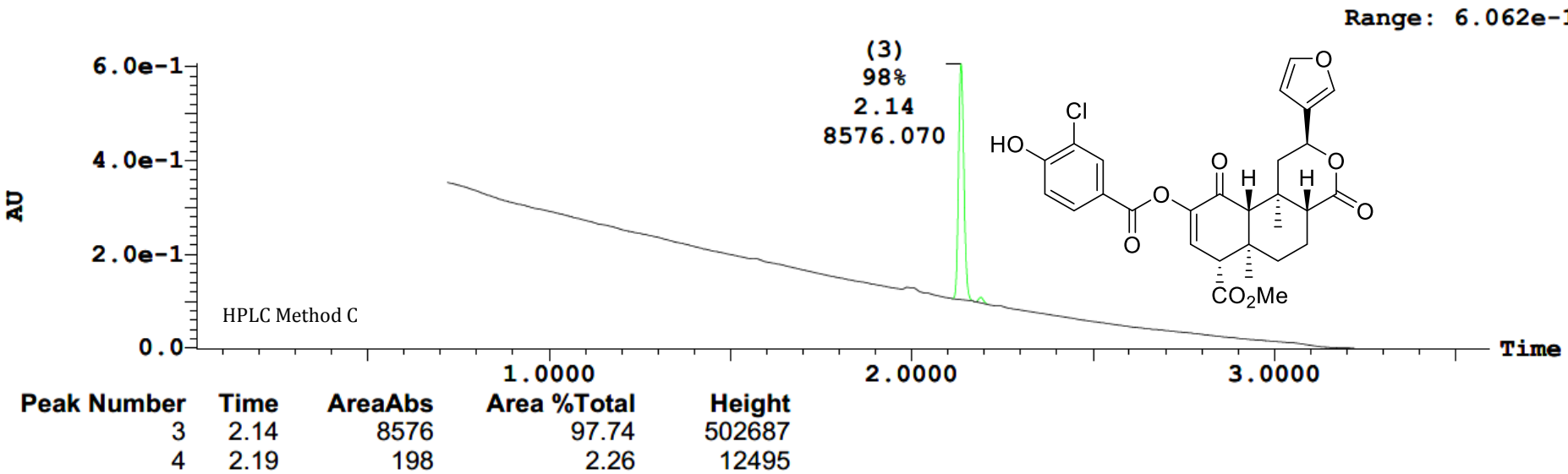

ID RMS-5-23 File RC170309WT008b Date 09-Mar-2017 Time 17:54:40 Description CCT138258

3: UV Detector: 214

2. $294 e-1$

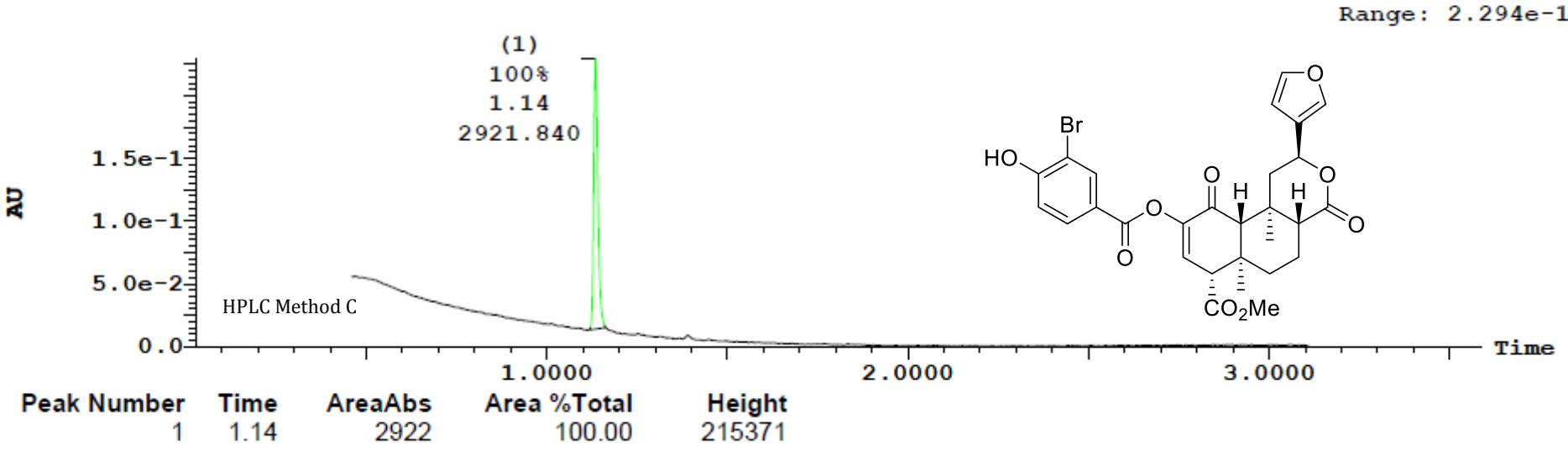

ID RMS-5-35 File RC170309WT011 Date 09-Mar-2017 Time 17:31:26 Description CCT138261

3: UV Detector: 214

$9.068 \mathrm{e}-1$

Range: $9.068 \mathrm{e}-1$

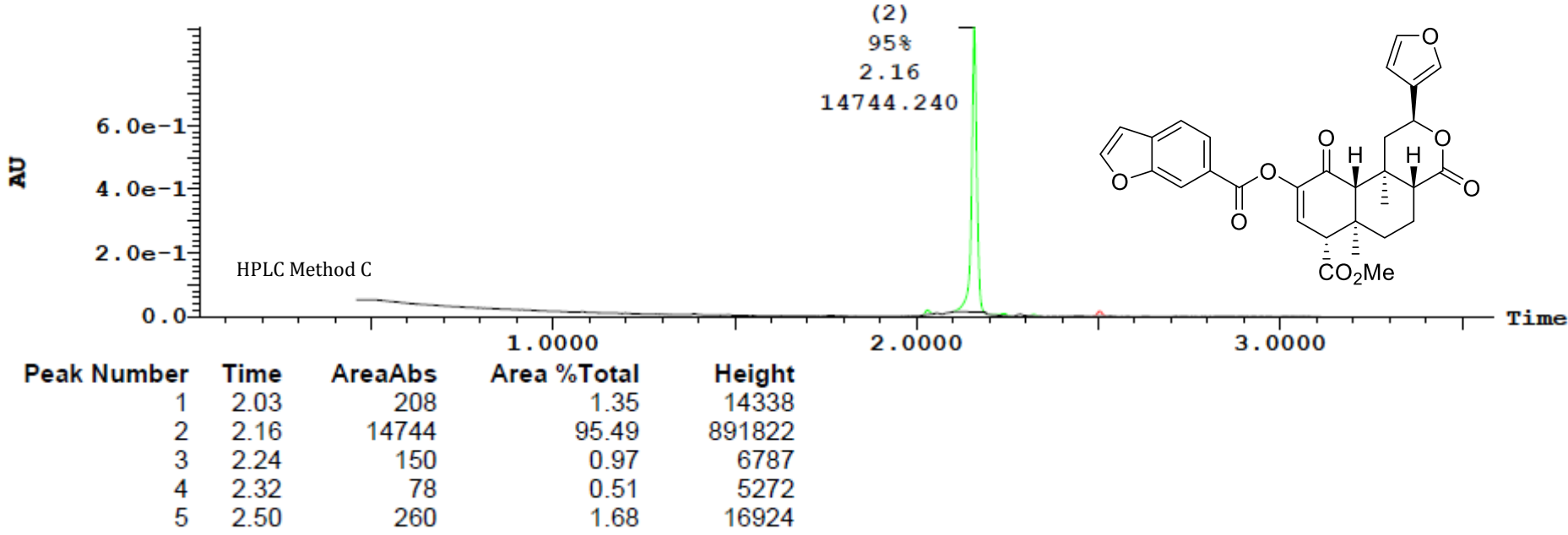


ID RMS-5-37 File RC170309WT014 Date 09-Mar-2017 Time 17:45:26 Description CCT138264

3: UV Detector: 214

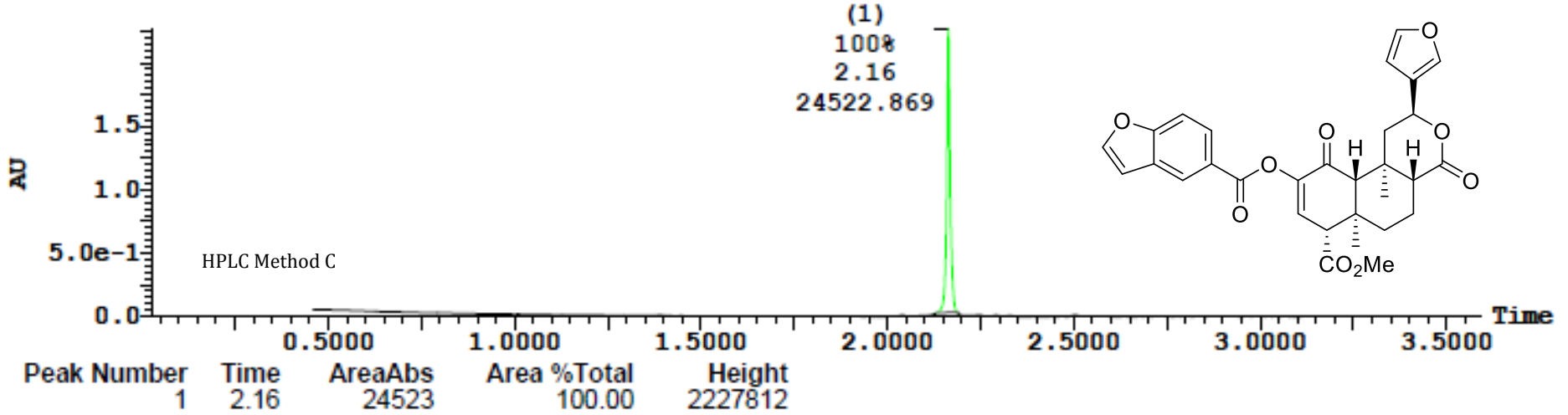

ID 4-CH2OH-Kurk File RC170421WT010 Date 24-Apr-2017 Time 16:10:10 Description CCT130538

3: UV Detector: 214

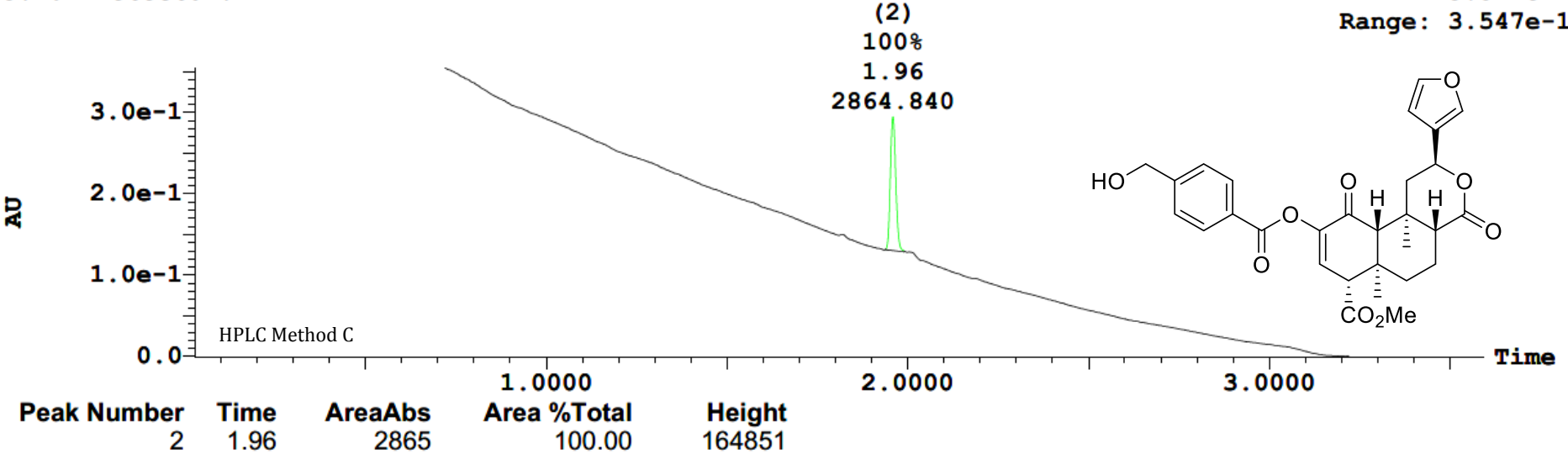

ID RMS-5-107 File RC170421WT011 Date 24-Apr-2017 Time 16:14:25 Description CCT130539

3: UV Detector: 214

$3.527 e-1$

Range: $3.527 \mathrm{e}-1$

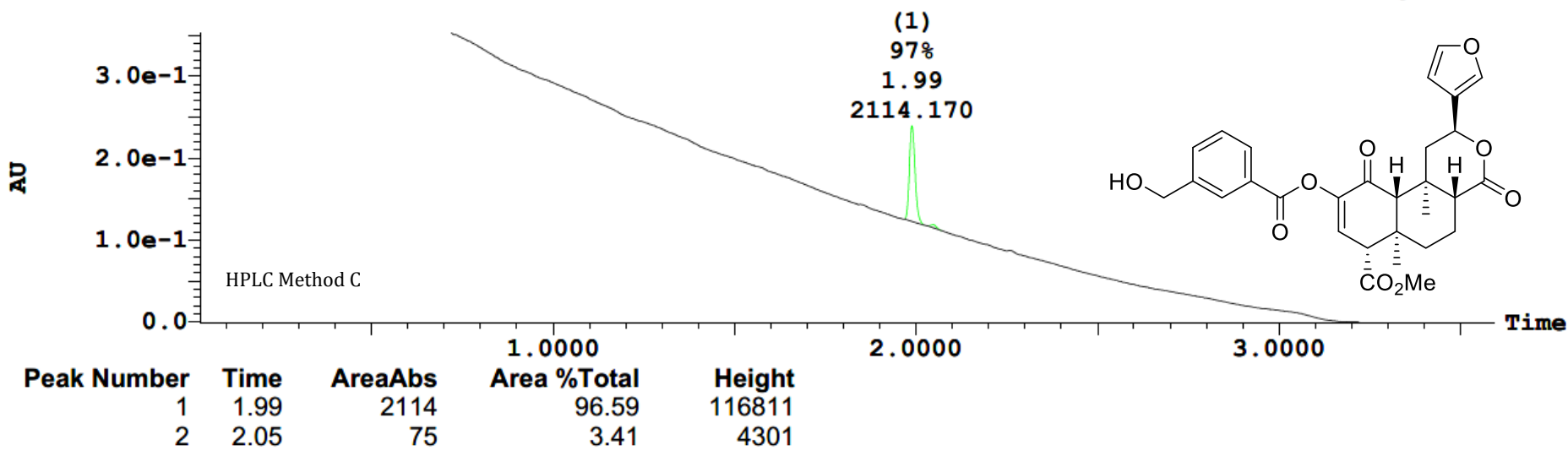




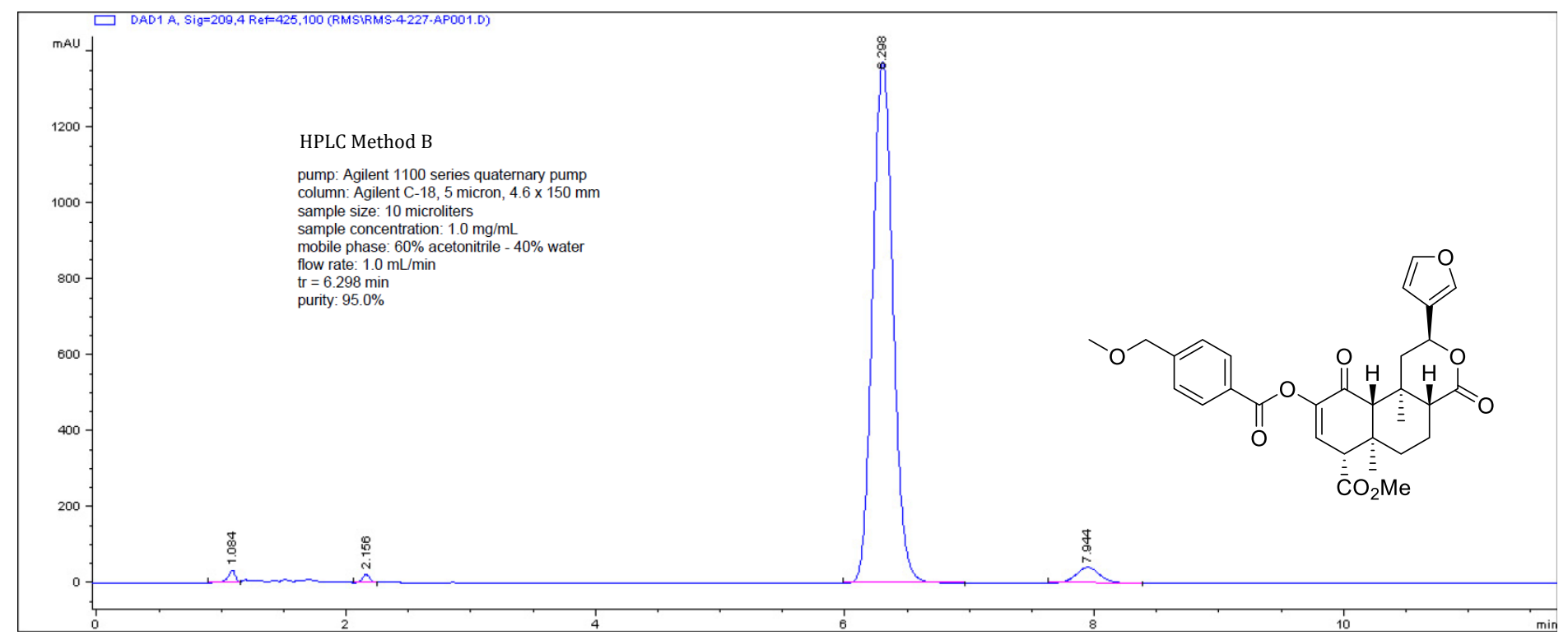




\section{References}

1. Simpson, D. S.; Katavic, P. L.; Lozama, A.; Harding, W. W.; Parrish, D.; Deschamps, J. R.; Dersch, C. M.; Partilla, J. S.; Rothman, R. B.; Navarro, H.; Prisinzano, T. E. Synthetic studies of neoclerodane diterpenes from Salvia divinorum: Preparation and opioid receptor activity of salvinicin analogues. J. Med. Chem. 2007, 50, 3596-3603.

2. Peng, Y. G.; Ji, C. Y.; Chen, Y. C.; Huang, C. Z.; Jiang, Y. Z. An efficient and selective deprotecting method for methoxymethyl ethers. Synth. Commun. 2004, 34, 4325-4330.

3. Arumugam, P.; Karthikeyan, G.; Perumal, P. T. A mild, efficient, and inexpensive protocol for the selective deprotection of TBDMS ethers using $\mathrm{KHSO}_{4}$. Chem. Lett. 2004, 33, 1146-1147.

4. Lampe, J. W.; Biggers, C. K.; Defauw, J. M.; Foglesong, R. J.; Hall, S. E.; Heerding, J. M.; Hollinshead, S. P.; Hu, H.; Hughes, P. F.; Jagdmann, G. E.; Johnson, M. G.; Lai, Y.-S.; Lowden, C. T.; Lynch, M. P.; Mendoza, J. S.; Murphy, M. M.; Wilson, J. W.; Ballas, L. M.; Carter, K.; Darges, J. W.; Davis, J. E.; Hubbard, F. R.; Stamper, M. L. Synthesis and protein kinase inhibitory activity of balanol analogues with modified benzophenone subunits. J. Med. Chem. 2002, 45, 2624-2643. 